University of Louisville

ThinkIR: The University of Louisville's Institutional Repository

Electronic Theses and Dissertations

7-2012

\title{
Electrokinetic colloid patterning characterization with a scanning laser.
}

Andrew Hardesty Work

University of Louisville

Follow this and additional works at: https://ir.library.louisville.edu/etd

\section{Recommended Citation}

Work, Andrew Hardesty, "Electrokinetic colloid patterning characterization with a scanning laser." (2012). Electronic Theses and Dissertations. Paper 1593.

https://doi.org/10.18297/etd/1593

This Master's Thesis is brought to you for free and open access by ThinkIR: The University of Louisville's Institutional Repository. It has been accepted for inclusion in Electronic Theses and Dissertations by an authorized administrator of ThinkIR: The University of Louisville's Institutional Repository. This title appears here courtesy of the author, who has retained all other copyrights. For more information, please contact thinkir@louisville.edu. 


\title{
ELECTROKINETIC COLLOID PATTERNING CHARACTERIZATION WITH A SCANNING LASER
}

\author{
By \\ Andrew Hardesty Work Jr. \\ B.S., University of Louisville, 2011

\begin{abstract}
A Thesis
Submitted to the Faculty of the University of Louisville

J. B. Speed School of Engineering as Partial Fulfillment of the Requirements for the Professional Degree
\end{abstract}

\section{MASTER OF ENGINEERING}

Department of Mechanical Engineering

July 2012 


\title{
ELECTROKINETIC COLLOID PATTERNING CHARACTERIZATION WITH A SCANNING LASER
}

\author{
Submitted by: \\ Andrew H. Work Jr. \\ A Thesis Approved On \\ (Date)
}

by the Following Reading and Examination Committee:

Dr. Stuart J. Williams, Thesis Director

Dr. Cindy Harnett

Dr. Yongsheng Lian 


\section{ACKNOWLEDGEMENTS}

My advisor, Dr. Stuart J. Williams, has been instrumental in my ability to complete this paper. He has sacrificed many valuable hours helping me get my bearings on this project and on graduate school in general. Vanessa Velasco also has spent numerous hours over the past year teaching me how to make devices and run tests. My professors, Dr. Hnat, Dr. Kelecy, and Dr. Lian, have also been very helpful over the past several years, and patient with me in answering questions both related to my thesis and to my general education. Finally, I would like to thank my wife for enduring with me during this last year all the while providing loving and patient support. 


\begin{abstract}
Rapid Electrokinetic Patterning (REP) is a relatively new method for collecting and manipulating micrometer-scale particles at an electrode surface. REP is an AC electrokinetic technique that uses induced fluid motion to capture and manipulate particles. A laser $(975 \mathrm{~nm})$ is focused on the surface of the parallel-plate electrode, generating a thermal gradient in the medium. This thermal gradient is acted upon by the AC electric field in such manner as to produce a vortex. Particles are trapped on the electrode surface at the center of the vortex. It is hypothesized that $\mathrm{AC}$ electroosmotic flows act to hold the particles to the electrode surface more strongly than other holding forces, such as dielectrophoresis (DEP).

The accumulation of single-size particles occurs in one layer, and is twodimensional in REP, and crystalline in nature under the correct conditions. Electrostatic forces separate the particles while fluid drag forces tend to corral the particles together. The particle aggregations tend to exhibit several exclusive, characteristic behaviors: the particles will group together very closely and uniformly, the particles will form arbitrary aggregations of tightly packed particles, or the particles will exhibit a nearly-random
\end{abstract}


spacing. Parameters are varied such that the average spacing is changed between the particles to transition the particles from nearly-random spacing to a uniform, tightly packed crystalline grid.

The relationship between the drag forces and the electrostatic forces parallel to the electrode surface are explored for different AC frequencies, AC voltages, laser powers, laser scan rates, and laser scan lengths. The effect these parameters have on the spacing of the particles is characterized, and aggregation crystallinity is discussed.

A more detailed force analysis is discussed for non-varying parameters with a dotshaped aggregation. The ability to use this force analysis for particle spectroscopy is briefly discussed. Particle motion was analyzed with Particle Tracking Velocimetry (PTV) using Matlab scripts. 


\section{TABLE OF CONTENTS}

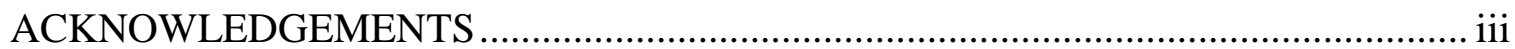

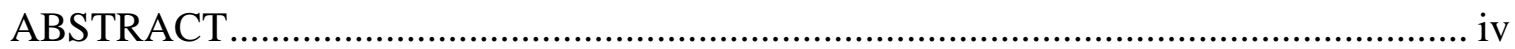

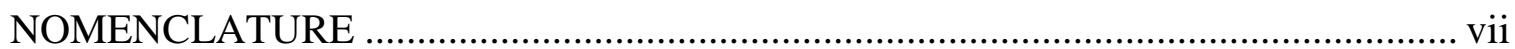

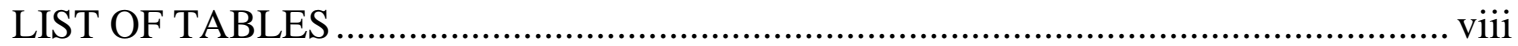

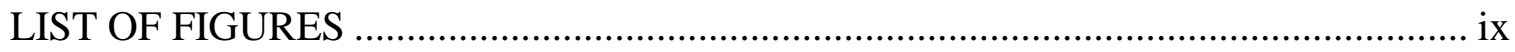

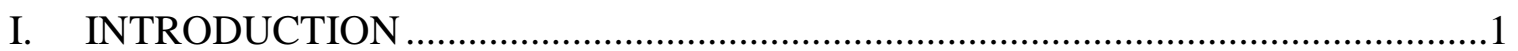

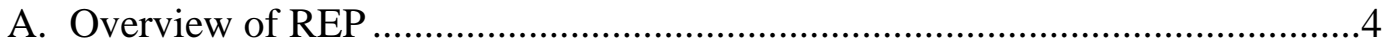

B. Overview of Relevant AC Electrokinetics......................................................5

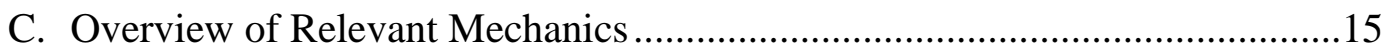

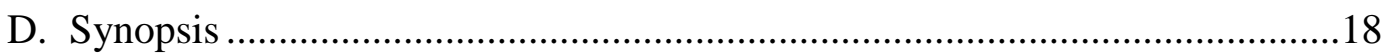

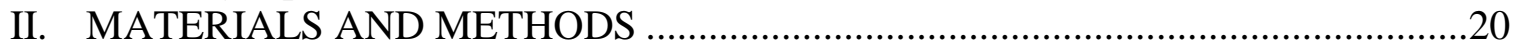

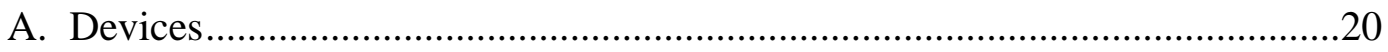

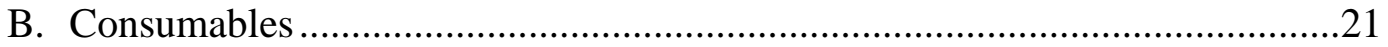

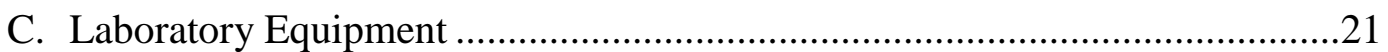

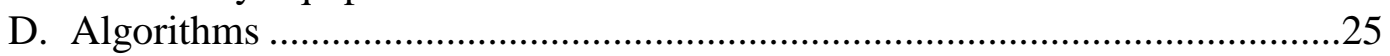

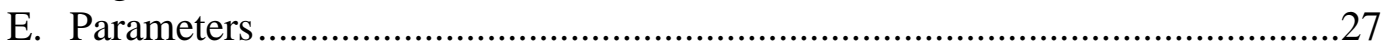

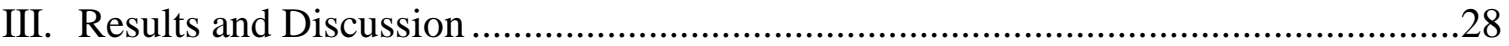

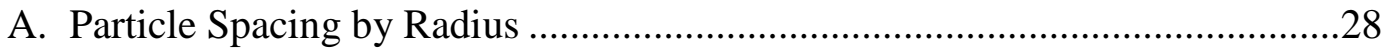

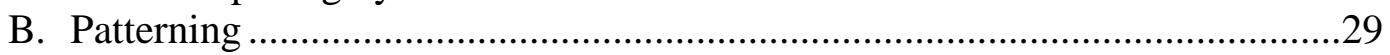

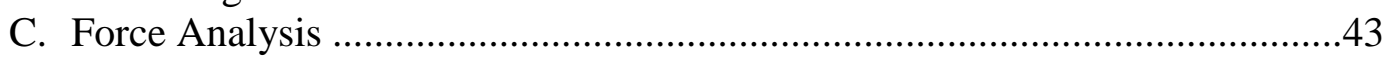

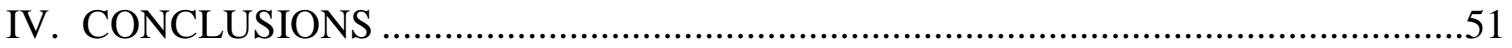

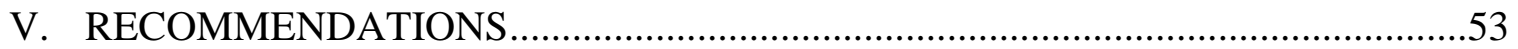

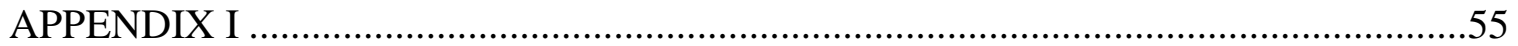

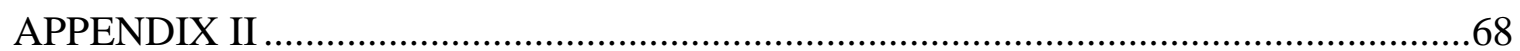

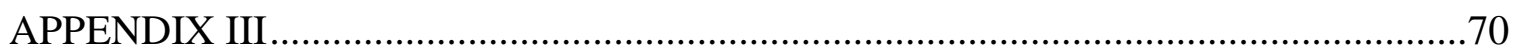

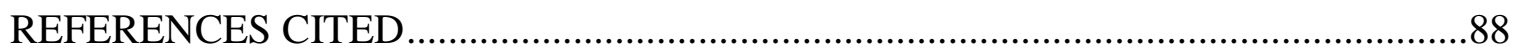

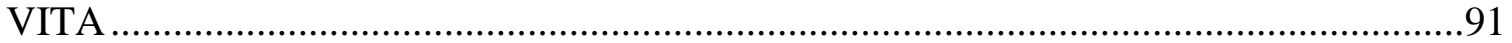




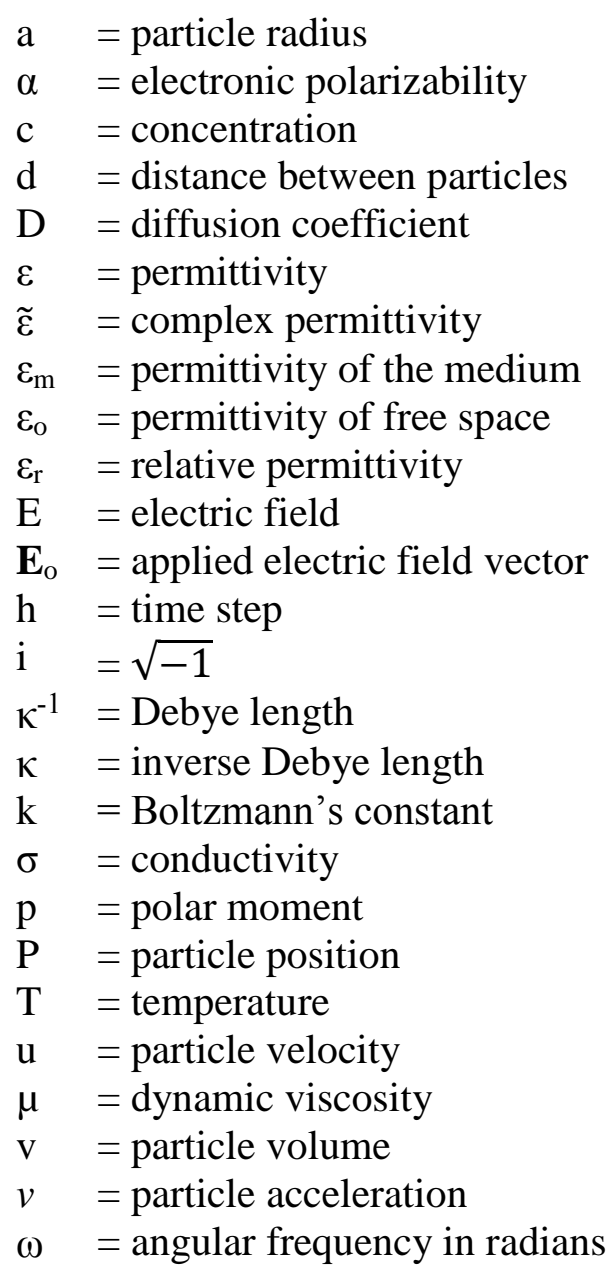




\section{LIST OF TABLES}

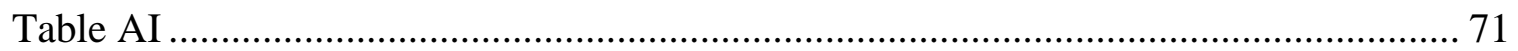

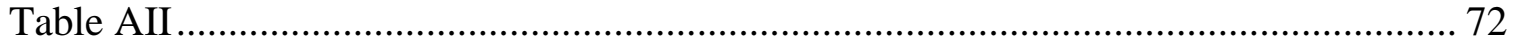

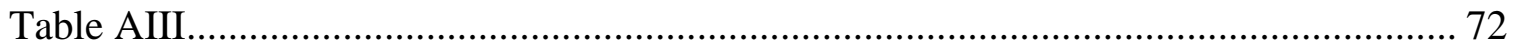

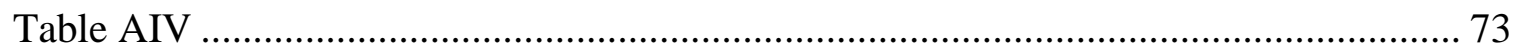




\section{LIST OF FIGURES}

FIGURE 1 - Setup of REP experiments, from (Kumar et al., 2010) …….......................... 4

FIGURE 2 - Ionic double-layer polarization seen in REP............................................. 11

FIGURE 3 - The Relationship of the Mechanics of REP............................................. 18

FIGURE 4 - REP device used for testing. .................................................................. 20

FIGURE 5 - Experimental REP set up, modified from (Velasco et al., 2012)................ 22

FIGURE 6 - Nikon Eclipse Ti microscope and other equipment. .................................... 22

FIGURE 7 - (a) Unprocessed image of dot aggregation. (b) Thresholded ........................ 24

FIGURE 8 - Sample particle selection...................................................................... 25

FIGURE 9 - Particle spacing versus radial position in a dot aggregation. ...................... 28

FIGURE 10 - Comparison of scanned line aggregations............................................... 29

FIGURE 11 - Average spacing versus AC frequency. .................................................... 32

FIGURE 13 - Average spacing versus laser scanning frequency.................................... 35

FIGURE 14 - Slow scanning speed time step, arrow indicating path of laser................... 36

FIGURE 15 - Two different, low scanning speeds................................................... 37

FIGURE 16 - Average spacing versus laser power with exponential fit.......................... 38

FIGURE 17 - Average spacing versus laser scanning length with linear fit. .................... 40

FIGURE 18 - Average spacing versus RMS AC voltage................................................ 42

FIGURE 19 - Five images of a released aggregation at $0.033 \mathrm{~s}$ intervals. ...................... 44

FIGURE 20 - Position and velocity of a released aggregation......................................... 44

FIGURE 21 - Velocity and acceleration of a released aggregation................................... 45

FIGURE 22 - Speed of a released aggregation by radial position.................................... 46

FIGURE 23 - Speed of a released aggregation by radial position.................................... 47

FIGURE 24 - Double-layer and Maxwell-Wagner force models. .................................... 48

FIGURE 25 - Scaled Maxwell-Wagner forces and drag forces. ...................................... 49

FIGURE A1 - Identified outer particles (circles) versus all particles ('x's)..................... 69

FIGURE A2 - Average spacing by radius in force experiments. .................................... 75

FIGURE A3 - Analysis data for experiment 1.......................................................... 76

FIGURE A4 - Analysis data for experiment 8............................................................ 77

FIGURE A5 - Analysis data for experiment 9......................................................... 78

FIGURE A6 - Analysis data for experiment 19....................................................... 79

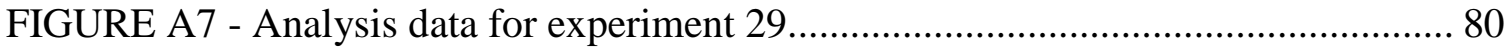

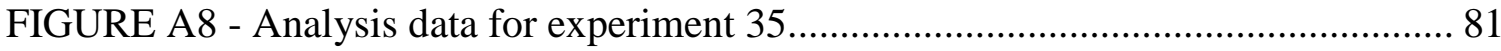

FIGURE A9 - Analysis data for experiment 36…………..................................... 82

FIGURE A10 - Analysis data for experiment 52 ...................................................... 83

FIGURE A11 - Analysis data for experiment 53 ....................................................... 84

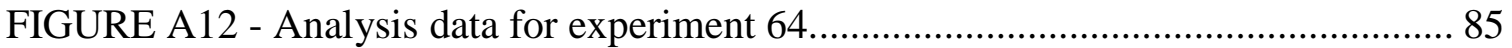

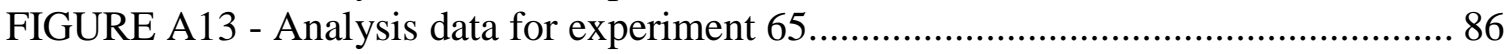

FIGURE A14 - Analysis data for experiment 69........................................................... 87 


\section{INTRODUCTION}

Trapping and manipulating colloids is the subject of a great amount of modern focus. Colloids are broadly defined as "any substance consisting of particles substantially larger than atoms or ordinary molecules but too small to be visible to the unaided eye..." (Encyclopædia Britannica, 2012b), and may be dispersed in another substance. The colloid and dispersant could both be the same phase (i.e. liquid dispersed in liquid, such as milk), or they could have differing phases (i.e. solids dispersed in liquids, such as blood). There are vast numbers of colloids that exist commercially, and colloids play a major role in research (Pieranski, 1983). Examples of colloids existing in the food industry include milk, butter, margarine, cheese, yoghurt, gelatin, and mayonnaise (Dickinson \& McClements, 1996). Aerosols are colloids solutions. In the medical field exists one of the most prominent solution containing colloids: blood. Blood cells, antibodies, and virus cells immersed in blood are colloids. Solids dispersed in liquids are sol colloids (Encyclopædia Britannica, 2012c), which is distinct from gels where the liquid medium is viscous enough to behave more like a solid. A sol type colloid where the fluid medium is water is a hydrosol, which is examined herein. Thus advances in colloid research affect a vast number of industries and modern health.

Uniform crystals are desirable in colloidal aggregations (Santana-Solano, Wu, \& Marr, 2006). Trapping and organizing particles is of significant interest in constructing nanostructure arrays using colloidal crystals, which is known as colloidal lithography (Zhang, Li, Zhang, \& Yang, 2010). The hexagonal close-packed nature of a colloidal aggregation has generated interest in the field of photonics (Jiang, Bertone, Hwang, \& Colvin, 1999). Jiang et al. used a colloidal crystal template to manufacture macroporous 
polymer membranes, which they then characterized. This demonstrates the usefulness of colloidal lithography using colloidal crystals. The same group later studied the optical properties of spherical shells and multilayer colloidal superlattices (Rengarajan, Jiang, Colvin, \& Mittleman, 2000; Rengarajan, Jiang, Larrabee, Colvin, \& Mittleman, 2001). They demonstrated that multi-layer colloidal crystals provide a method for providing an optical stop band. Photonic crystals require a length scale in the sub-micron range, for which REP is well suited.

Various techniques have been developed to trap and manipulate particles. Rapid Electrokinetic Patterning (REP) is a relatively new method of collecting and organizing particles on an electrode surface (Williams, Kumar, \& Wereley, 2008), and is thermoelectrokinetic in nature. Other techniques include optical trapping (Novotny, Bian, \& Xie, 1997), electrophoresis (Rodriguez \& Armstrong, 2004), dielectrophoresis (Kim, Asmatulu, Marcus, \& Papadimitrakopoulos, 2011; Zhou, White, \& Tilton, 2005), thermophoresis (Zheng, 2002), and sedimentation (Colvin, 2001).

REP exhibits a number of advantages over other techniques. Unlike traditional optical trapping methods, which can only trap a few particles, REP is well suited to handle a large number of particles. Unlike electrophoretic techniques, REP and DEP can move neutral particles. Unlike many electrophoretic and dielectrophoretic (DEP) methods, REP does not require complicated electrode geometry; on the contrary, it requires simple (parallel plate) geometry. REP also allows a great degree of freedom in controlling the particles. REP relies on thermal gradients to produce bulk forces and induce vortices, which can be dynamically and remotely controlled with a laser (removing the need for intrinsic heating elements). This allows the particles to be moved 
across the surface of the electrode, and the shape and spacing of the aggregation to be changed rapidly. Most DEP techniques require fixed electrodes with comparatively complicated geometry. They are limited by the fixed nature of their electrodes. The aggregation of particles is also frequency dependent, providing a further degree of freedom in tuning an REP system (Williams et al., 2008). Thus REP is simplistic in its implementation and powerful in its application.

As REP is relatively new, its primary disadvantage lies in its lack of development and characterization. The fundamental nature of its mechanics are known and the technique is useful for trapping and moving groups of particles, but there are many insufficiently-known mechanics such as the nature of particle-particle spacing, the microvortex corralling the particles, and the holding forces that trap the particles at the electrode surface. This paper explores several of these mechanics by studying the drag and electrostatic forces on the particles, as well as the nature of the aggregations. The ability to form, pattern, control, and characterize crystallinity within particle aggregations is also discussed. The spacing of particles is important to characterize the crystallinity of the aggregation. As particle spacing approaches a minimum, the particles will form a crystalline grid.

Crystallinity can be judged by the number of neighboring particles 'touching' each individual particle (Kim et al., 2011), but more relevant to REP is the spacing between each particle and its' neighbors. REP can dynamically control the spacing of particles within an aggregation, and can spontaneously form a 2D crystal. This work investigates particle-particle distances within the whole aggregation to characterize its crystallinity for various REP parameters. 


\section{A. Overview of REP}

REP uses two parallel-plate electrodes. Herein, one plate is transparent for visual observation. Water with a specified $\mathrm{KCl}$ molarity is placed between the plates. The goal of REP is to bring the spherical particles to the surface of an electrode, and organize them in a manner useful to their study or application. This thesis focuses on the dynamics of the particle aggregations and on their application.

REP has two power sources: a laser and an AC electric field. The laser's role is solely to create thermal gradients in the medium, while the AC field serves a number of functions. The AC field is necessary to both induce fluid motion by acting on the medium through thermal gradients, and also to trap the particles at the surface of the electrode. Figure 1 shows the experimental setup used in REP.

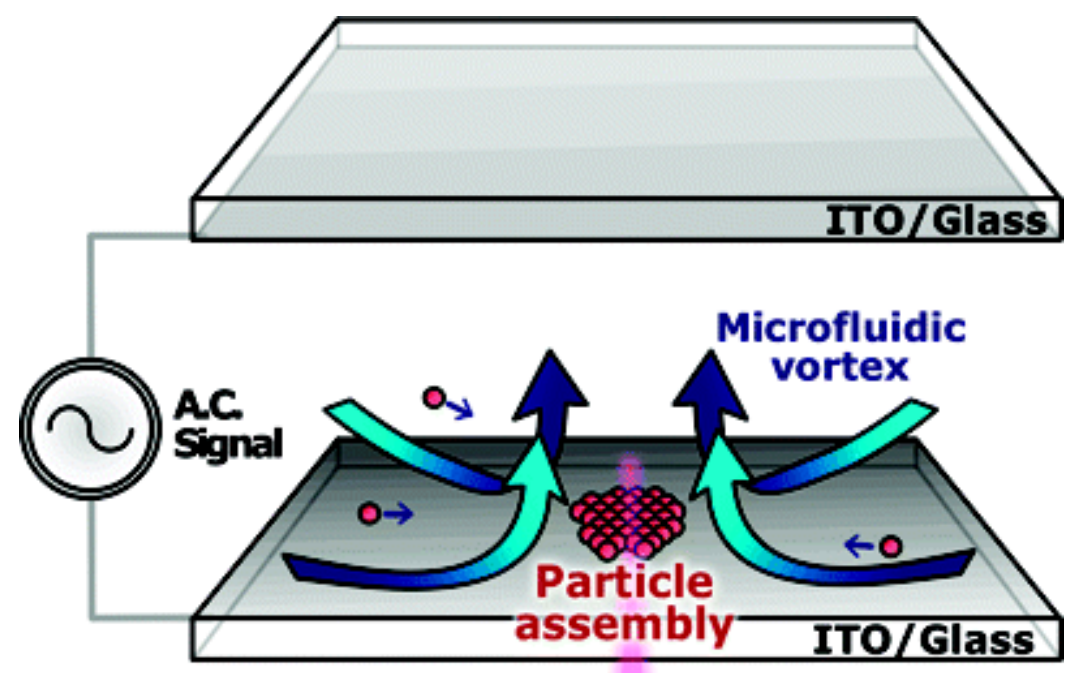

Illumination

\& Viewing

FIGURE 1 - Setup of REP experiments, from (Kumar et al., 2010). 
The mechanisms required for REP are as follows. The laser hits and is absorbed by the bottom ITO surface (being focused through the viewing objective). The induced thermal non-uniform field generates a spatial gradient in the electric and dielectric properties of the medium. The AC field acts on this gradient to create a bulk force. This force creates an axisymmetric vortex centering at the laser spot (see Figure 1). The induced vortex carries suspended particles and brings them in near-contact with the surface. Dielectrophoretic forces and other electrokinetic mechanisms such as AC electroosmosis (ACEO) hold the particles adjacent to the electrode surface (Fagan, Sides, \& Prieve, 2002, 2005; Zhou et al., 2005). Drag forces from the vortex corral the particles to the center of the vortex, while additional electrokinetic forces repel the particles away from each other. The shape and location of the vortex can be controlled with the scanning laser. As shown in Figure 1, the laser is focused on the lower ITO surface through the optics on the microscope used to observe fluid motion.

Important to understanding ACEO are electrokinetic particle relaxation mechanisms. The particle-electrode holding forces are not well understood (Fagan et al., 2005). Many papers on the subject of colloidal sorting focus on the lateral forces acting on particles. In this case, the dominant particle-electrode holding force is hypothesized to be due to ACEO; however, this is not investigated. These lateral electrokinetic forces will be discussed in greater detail in the following sections.

\section{B. Overview of Relevant AC Electrokinetics}

Fundamental to REP is dielectrophoresis. Dielectrophoresis is the force on a dielectric material due to a gradient in the electric field. Positive dielectrophoresis occurs when the material of interest moves towards the stronger field; negative dielectrophoresis 
occurs when the material moves away from the stronger field. The electric field in REP is uniform, but both the presence and polarization of the particles disturb the uniform nature of the field. The presence of the particle changes the electric field, so it will either tend to pass through or around the particle creating a local non-uniformity. The polarization disturbs the field due to the charges on the particle producing their own field, superposed over the uniform field. It is not known which, if either, of these effects are significant.

Electronic polarizability $(\alpha)$ is the ability of a dielectric material to polarize when subjected to an electric field. A polarized particle is one in which positive and negative charges separated to form electric poles, in this case, a dipole. The polar moment of a particle is given by (Lyklema, 1991)

$$
\mathrm{p}=\mathrm{qd}=\mathrm{v} \tilde{\alpha} \mathrm{E}
$$

where $\mathrm{q}$ is the charge at each end of the dipole, $\mathrm{d}$ is the distance between two poles, $\mathrm{v}$ is the volume of the particle, $\tilde{\alpha}$ is the effective polarizability of the particle, and $\mathrm{E}$ is the electric field applied to the particle.

There are several particle polarization mechanisms, including the movement of ions (atomic polarization), the shifting of an electron cloud (electronic polarization), or the rotation of permanent dipoles (such as the orientation of a water molecule). Polarization mechanisms take a finite amount of time to occur and each have a characteristic frequency response. These particular polarization mechanisms occur on a very small scale compared to the size of the particles used herein, and relax at $\mathrm{AC}$ frequencies larger than those used in REP. Orientational polarization typically relaxes around $10^{9} \mathrm{~Hz}$, atomic polarization around $10^{12} \mathrm{~Hz}$, and electronic polarization around 
$10^{15} \mathrm{~Hz}$ (Agilent, 2006). Of specific interest are polarization mechanisms on the scale of the particle itself, which are useful for particle trapping.

Two polarization mechanisms are particularly important: Maxwell-Wagner interfacial polarization, and ionic double layer polarization. These occur on the size scale of the particle, and relax lower frequencies than other polarization mechanisms. This is due to the length scale of the polarization being on the order of the particle itself. Maxwell-Wagner interfacial polarization is important as it is a significant factor in dielectrophoretic forces that are capable of attracting or repelling particles to/from areas of high electric field gradients. Ionic double layer polarization is important because it plays a key role in AC electro-osmosis (ACEO), an electro-hydrodynamic mechanism capable of trapping particles against the electrode surface in REP. Both of these topics are very involved; a simplified case is presented and used in analysis for REP. These mechanisms are explored herein to the extent necessary to grant a sufficient understanding of REP. For a more detailed presentation, see (Kirby, 2010; Lyklema, 1991; Munson, 2009).

The focus of the following two sections is on the relaxation frequency for each respective polarization mechanism. These relaxation frequencies are important to distinguish which mechanism is involved with REP to hold the particles. Knowing which mechanism is holding the particles is important to determine the dipole moment on each particle. It is the hypothesis of the author that the double layer polarization is responsible for holding particles to the surface of the electrode, and that interfacial polarization is responsible for separating the particles. This was not further investigated. The function 
that each polarization mechanism serves has not been thoroughly explored, and holds potential for future work expanding knowledge of REP.

\section{Maxwell-Wagner Interfacial Polarization}

Interfacial polarization involves the inhibited movement of charge carriers (Agilent, 2006). Charge carriers are collected at the interface of two different dielectric mediums with different permittivities and/or conductivities (Morgan \& Green, 2003). This is true regardless of the geometry of the system, though the nature of polarization would be different. The Maxwell-Wagner interfacial polarization is due to this surface charge. At low frequencies, charge carriers have plenty of time to accumulate, while at higher frequencies the charge carriers do not have enough time to accumulate at the surface of the particles, and the polarization relaxes. The dipole moment of a spherical colloidal particle was calculated using (Morgan \& Green, 2003)

$$
\mathrm{p}=3 \varepsilon_{\mathrm{m}}\left(\frac{\widetilde{\varepsilon}_{\mathrm{p}}-\widetilde{\varepsilon}_{\mathrm{m}}}{\tilde{\varepsilon}_{\mathrm{p}}+2 \widetilde{\varepsilon}_{\mathrm{m}}}\right) \mathrm{vE}=3 \varepsilon_{\mathrm{m}} \mathrm{K}_{\mathrm{cm}}
$$

where $\varepsilon_{\mathrm{m}}$ is the permittivity of the medium, $\widetilde{\varepsilon}_{\mathrm{p}}$ and $\widetilde{\varepsilon}_{\mathrm{m}}$ are the complex permittivities of the particle and the medium, respectively, and $\mathrm{K}_{\mathrm{cm}}$ is the Clausius-Mossotti factor. The complex permittivity is defined as

$$
\tilde{\varepsilon}=\varepsilon_{\mathrm{o}} \varepsilon_{\mathrm{r}}-\frac{\mathrm{i} \sigma}{\omega}
$$

where $\varepsilon_{0}$ is the permittivity of free space, $\varepsilon_{\mathrm{r}}$ is the relative permittivity, $\mathrm{i}$ is $\sqrt{-1}, \sigma$ is conductivity, and $\omega$ is the frequency of the electric field in radians. Combining (1) and (2), the effective polarizability of a homogeneous colloidal particle is given by

$$
\tilde{\alpha}=3 \varepsilon_{\mathrm{m}} \mathrm{K}_{\mathrm{cm}}
$$


As such, the Clausius-Mossotti factor is directly proportional to the effective polarizability.

This form of polarization is important to REP as dielectric forces act on the particle due to the increased electric field (from the charge build up at the interface and the presence of the particle), and attract the particles to the electrode surface under positive dielectrophoresis. The particle disturbs the field, creating non-uniformities. These fields interact with the field produced by the electrodes, and make them stronger or weaker; this causes DEP forces to affect the particle. The time-averaged DEP force acting on the particle are given using (Morgan \& Green, 2003)

$$
\left\langle\mathrm{F}_{\mathrm{DEP}}\right\rangle=\pi \mathrm{a}^{3} \varepsilon_{\mathrm{m}} \operatorname{Re}\left[\mathrm{K}_{\mathrm{cm}}\right] \nabla|\mathrm{E}|^{2}
$$

where $\mathrm{a}$ is the particle radius. The real part of the Clausius-Mossotti factor will determine whether the DEP force is positive or negative, which determines if the particle will be attracted to or repelled from the electrode surface.

The induced dipole in each particle will also create a repulsive force between neighboring particles. The force between two neighboring spherical particles with an induced dipole is approximated to an order of magnitude using (Nadal, Argoul, Hanusse, Pouligny, \& Ajdari, 2002)

$$
\mathrm{F}_{\mathrm{e}} \approx \frac{3 \pi \varepsilon_{\mathrm{m}} \mathrm{a}^{6} \mathrm{E}_{\mathrm{o}}^{2}}{\mathrm{~d}^{4}}
$$

where $E_{o}$ is the applied electric field, and $d$ is the distance between the neighboring particles. For more information on DEP, see (Kirby, 2010; Morgan \& Green, 2003). 


\section{Ionic Double-Layer Polarization}

Ionic double-layer polarization is similar to Maxwell-Wagner Interfacial Polarization in that it involves ions being trapped at the surface of the colloidal particle. Rather than DEP forces bringing ions to the surface of the colloidal particle, electrostatic forces attract ions. In ionic double-layer polarization, the colloidal particles gain an electric charge from the solution in which they are submerged. The particles may absorb molecules from the medium or chemical groups on the particle's surface may disassociate, giving the particle a net charge. This surface charge attracts ions with an opposite charge. These ions form two layers around the particle: a thin layer strongly attracted to the surface (the Stern layer, or the bound layer), and a thicker layer (the diffuse layer, or Gouy-Chapman layer) that is less-strongly attracted to the particle. These two layers are the 'double layer', or electric double layer (EDL), around the particle, and will polarize by moving around the perimeter of the particle. Figure 2 is a depiction of ionic double-layer polarization. 


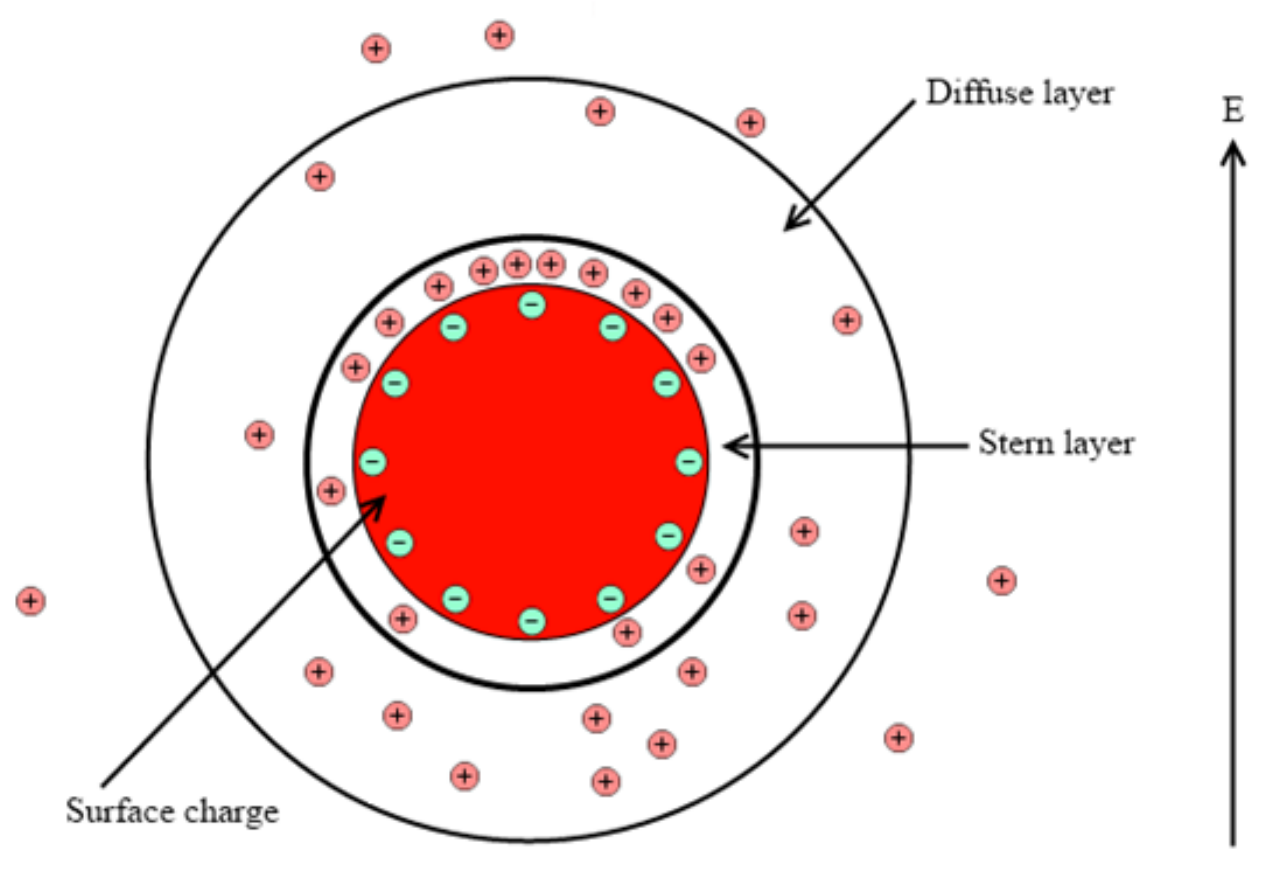

FIGURE 2 - Ionic double-layer polarization seen in REP.

As shown in Figure 2, the Stern layer is much closer to the particle than the diffuse layer, and, as such, has much lower mobility. As the diffuse layer has higher mobility, it polarizes faster than the Stern layer, and increases the speed at which the Stern layer polarizes above that predicted by the Schwarz model (Morgan \& Green, 2003). The orientation of the dipole may be dependent on the properties of the medium and the applied field. The mobility of the Stern layer and the mobility of the diffuse layer are not constant, so the particle may polarize in either direction. The amount of surface charge in each layer is unknown, but combined are equivalent to the total surface charge on the particle (effectively reducing the total charge of the particle to zero). The order of the relaxation time of the Stern layer was calculated using Schwarz's model,

$$
\tau=\frac{\mathrm{a}^{2}}{2 \mathrm{D}}
$$


where $\mathrm{D}$ is the diffusion coefficient. This model does not include the effects of the diffuse layer. This also does not include ion exchanges between the suspending electrolyte and the charged layer (Morgan \& Green, 2003). Ionic double layer polarization is important to ACEO as it provides a mechanism for inducing fluid motion around a particle, discussed in the next section.

The Debye length is the characteristic length of the EDL, and describes the distance from a charged particle it takes for the potential to fall to a fraction of the surface potential equivalent to $\mathrm{e}^{-1}$. For an aqueous potassium chloride $(\mathrm{KCl})$ solution, the Debye length is given with respect to molar concentration, c, as (Morgan \& Green, 2003)

$$
\kappa^{-1}=1.764 \times 10^{-11} \sqrt{\frac{\mathrm{T}}{\mathrm{c}}}
$$

where $\mathrm{T}$ is temperature. For the aqueous $\mathrm{KCl}$ solution used $(0.13 \mathrm{mM}), \kappa^{-1}$ corresponded to a length of $\sim 26 \mathrm{~nm}$. The Debye length is important as simplifications can be made to the electrokinetic model for a small Debye length (when compared to the radius of the particle).

The force between two spherical particles due to the EDL is approximated using DLVO theory, named for Derjaguin, Landau, Verwey, and Overbeek (Morgan \& Green, 2003),

$$
\mathrm{F}_{\mathrm{R}} \approx \frac{2 \pi \mathrm{a} \sigma_{\mathrm{qd}}^{2}}{\kappa \varepsilon_{\mathrm{m}}} \mathrm{e}^{-\kappa \mathrm{d}}
$$

where $\sigma_{\mathrm{qd}}$ is the diffuse layer charge density, and $\kappa$ is the inverse Debye length. Since the force is proportional to $\mathrm{e}^{-\mathrm{kd}}$, and the inverse Debye length is a very large number (on the 
order of $10^{9}$ ), this force scales down very rapidly. DLVO theory includes attractive van der Waals interaction, and repulsive double layer interaction.

\section{3. $\underline{\text { AC Electroosmosis }}$}

ACEO in REP is the result of ion motion across the surface of particles, specifically from the diffuse layer of the EDL. As the ions move across the surface, viscous effects carry with them the surrounding medium. A non-uniform electric field is required for ACEO just as it is required for DEP (Morgan \& Green, 2003). Near the surface of an electrode, the particle will create non-uniformities in the electric field. When ACEO occurs near the surface of an electrode, ions will move tangentially to the surface of the electrode as well as along the edge of the particles. As a result, a localized vortex is created between the particles and the electrode. This vortex lowers local pressure, which tends to pull the particle towards the electrode. Furthermore, fluid flow produced by ACEO is frequency dependent, and has a Gaussian-shaped profile (Morgan \& Green, 2003).

The particles in REP are observed to move randomly. It is the hypothesis of the author that this is due to Brownian motion and/or ACEO; however, this is not investigated further. As discussed in section $\mathrm{C} 3$, the particles store relatively little kinetic energy in their motion. As a result, fluid drag would quickly dampen movement due to electrostatic forces. ACEO is therefore suspected to cause this 'random' movement, which provides a challenge for proper analysis (as discussed in Results and Discussion, section C3 with respect to spectroscopy).

ACEO also pulls particles together over short distances. Under particular conditions, particles will form arbitrary aggregations on the surface of an electrode within 
a single aggregation. This effect has been demonstrated at low frequencies, and occurred because of electrohydrodynamic flow (Ristenpart, Aksay, \& Saville, 2004). This attractive force is present at higher frequencies, but repulsive forces begin to dominate as the frequency increases. This effect demonstrates that ACEO can generate force on the particles at the frequencies of interest. The phenomenon of particles grouping into arbitrary aggregations at low frequencies is not further discussed, however, and is outside the scope of this thesis. For more information on ACEO see (Kirby, 2010; Morgan \& Green, 2003).

\section{Thermolelectrokinetic Forces Overview}

The heat from the laser being absorbed by the ITO electrode surface is dissipated to the medium. This creates a thermal gradient in the medium, which, in turn, creates a gradient in the permittivity and conductivity of the medium. This force was approximated using (Lyklema, 1991)

$$
\left\langle\mathrm{f}_{\mathrm{e}}\right\rangle \approx \frac{1}{2} \operatorname{Re}\left(\frac{\sigma \varepsilon_{\mathrm{m}}\left(\alpha_{\mathrm{e}}-\beta\right)}{\sigma+\mathrm{i} \omega \varepsilon_{\mathrm{m}}}\left(\nabla \mathrm{T} \cdot \mathbf{E}_{\mathrm{o}}\right) \mathbf{E}_{\mathrm{o}}^{*}-\frac{1}{2} \varepsilon_{\mathrm{m}} \alpha_{\mathrm{e}}\left|\mathbf{E}_{\mathrm{o}}\right|^{2} \nabla \mathrm{T}\right)
$$

where * signifies the complex conjugate, and

$$
\alpha_{\mathrm{e}}=\frac{1}{\varepsilon_{\mathrm{m}}}\left(\frac{\delta \varepsilon_{\mathrm{m}}}{\delta \mathrm{T}}\right) \text { and } \beta=\frac{1}{\sigma}\left(\frac{\delta \sigma}{\delta \mathrm{T}}\right)
$$

In an aqueous $\mathrm{KCl}$ solution, $\alpha_{\mathrm{e}}$ is approximately $-0.4 \% \mathrm{~K}^{-1}$, and $\beta$ is approximately $2 \%$ $\mathrm{K}^{-1}$ (Lide, 1993). The first term in (10) is the Coulomb force, while the second is the dielectric force. Since these two forces have the potential to act in opposite directions, and since the dielectric force will dominate as the AC frequency increases, the direction of flow could be controlled with the frequency. It does not reverse in REP, however. 


\section{Overview of Relevant Mechanics}

Also important to REP are the mechanics involved with particles suspended in water. The following sections discuss the fluid dynamics associated with the particles, the thermal dissipation in the chip, and additional mechanics such as gravity and Brownian motion.

\section{Fluid Dynamics and Drag}

Important to the study of colloids under the influence of a micro-vortex is fluid dynamics. Of particular interest is the behavior of the particles at the surface of the electrode. A particle at the surface of an electrode will experience lift (normal to the plane of the electrode), and drag (perpendicular to lift). The lift force, while relevant to REP, is not pertinent to the topic herein. The drag force is of primary importance. Fluid drag will both corral the particles into a group and inhibit outward movement when the laser is deactivated.

Stokes' Law was used to determine the drag on particles as they were released (laser deactivated). Stokes' Law is valid for flows with Reynolds numbers that are much less than one. In REP, the maximum particle velocity is on the order of $100 \mu \mathrm{m} / \mathrm{s}$. For 1 $\mu \mathrm{m}$ particles, this corresponds to a Reynolds number on the order of $10^{-4}$. Drag was calculated using (Munson, 2009)

$$
f_{d}=6 \pi \mu u a
$$

where $\mu$ is the dynamic viscosity of the medium, and $u$ is the particle velocity. In order to use Stokes' Law for REP, it must be assumed that the medium's velocity is static. The particles do not touch the surface of the electrode (Fagan, Sides, \& Prieve, 2004), and can be treated as being suspended in the medium; however, boundary layer effects from the 
electrode surface and fluid medium velocity are still of concern. Effects due to the boundary layer are minimized by only using the first images after the particles are released, spanning a duration of up to 0.2 seconds.

\section{Thermal Dissipation and Time Response}

Of primary importance to colloidal patterning is the time constant associated with the dissipation of heat produced by the laser. This was studied by (Velasco, Work, \& Williams, 2012). Velasco et al. used Rhodamine B, a temperature-dependent fluorescent dye, to measure the temperature of the substrate. They also demonstrated that the temperature increase was a function of both laser power and scanning speed; i.e. if the laser were scanned over a specified length, the maximum temperature obtained would be less than that if the laser were fixed at a single point.

Velasco et al. further demonstrated an averaged velocity plot for both a single point and a scanned line at the same laser power. The scanned point, having a larger gradient, produced larger fluid velocities. For scanning lines, as the speed of the scan increased, the maximum velocity decreased. As speed increased, temperature variation across the scanned area decreased. This decreased the maximum thermal gradient, and thereby decreased the maximum fluid velocity.

\section{Gravitational/Buoyant Forces and Acceleration}

Buoyancy effects on each particle are neglected in calculations. They are both very small, in proportion to relevant forces, and in the direction normal to the surface of the electrode (as is the lift force). The density of polystyrene is close to that of water $\left(\sim 1050 \mathrm{~kg} / \mathrm{m}^{3}\right.$ (Scientific, 2012) compared to $\sim 998 \mathrm{~kg} / \mathrm{m}^{3}$ (Munson, 2009)). The magnitude of these forces is small due to the size scale of the particle and its density. The 
drag force parallel to the surface of the electrode is on the order of $10^{-7} \mathrm{~N}$, whereas the difference in the gravitational and buoyant forces is approximately $2.05 \cdot 10^{-15} \mathrm{~N}$. Due to the small magnitude of the gravitational force, changes in the orientation of the chip do not cause the aggregation of the particles to appreciably change. Since the mass of each particle is small $\left(\sim 4.4 \cdot 10^{-15} \mathrm{~kg}\right)$, the particles accelerate very rapidly. Image capture was limited to a maximum of $30 \mathrm{~Hz}$; before one frame is recorded, the particle has essentially reached its terminal velocity. The magnitude of the observable acceleration was on the order of $10^{-3} \mathrm{~ms}^{-2}$, meaning the net force on the particles is on the order of $10^{-17} \mathrm{~N}$. As the drag is on the order of $10^{-7} \mathrm{~N}$, the net force can be treated as zero.

\section{Brownian Motion}

Brownian motion is the random movement of molecules in a fluid (Encyclopædia Britannica, 2012a). The movement of the particles is apparently random, preferring no one direction over the other. The rms displacement of a suspended particle due to Brownian motion can be determined using the diffusion coefficient, given from the Stokes-Einstein equation as (Dunstan \& Stokes, 2000)

$$
\mathrm{D}=\frac{\mathrm{kT}}{6 \pi \mu \mathrm{a}}
$$

where $\mathrm{k}$ is Boltzmann's constant. This is related to the rms displacement using

$$
\mathrm{x}_{\mathrm{rms}}=\sqrt{2 \mathrm{Dt}}
$$

The rms displacement corresponding to a micron-size sphere in water at $293 \mathrm{~K}$ over $1 / 30^{\text {th }}$ of a second is $120 \mathrm{~nm}$. This may be directly responsible for the random movement demonstrated by the colloidal particles in REP. 


\section{Vortex Shedding}

Vortex shedding doesn't affect the colloidal particles used in REP due to the low Reynolds numbers involved. Vortex shedding occurs at Reynolds numbers above 300 (Williamson, 1988). The order of magnitude of the Reynolds number in REP is $10^{-4}$, indicated vortex shedding does not affect the particles.

\section{Synopsis}

Colloid science affects a great number of fields, including biology and life sciences, environmental sciences, petroleum sciences, imaging technology, commercial applications, and more (Hiemenz, 1997). The ability to study colloids therefore garners a significant amount of attention from a number of disciplines and industries. There are many techniques to capture colloids for observation, out of which REP stands out for its simplistic implementation and high order of controllability. REP, however, uses many different mechanics and is difficult to understand. Furthermore, many fundamental concepts to REP are not well understood, such as the nature of the polarization mechanisms and the effect ACEO has on the particles. The relationships between different areas of REP are depicted in Figure 3.

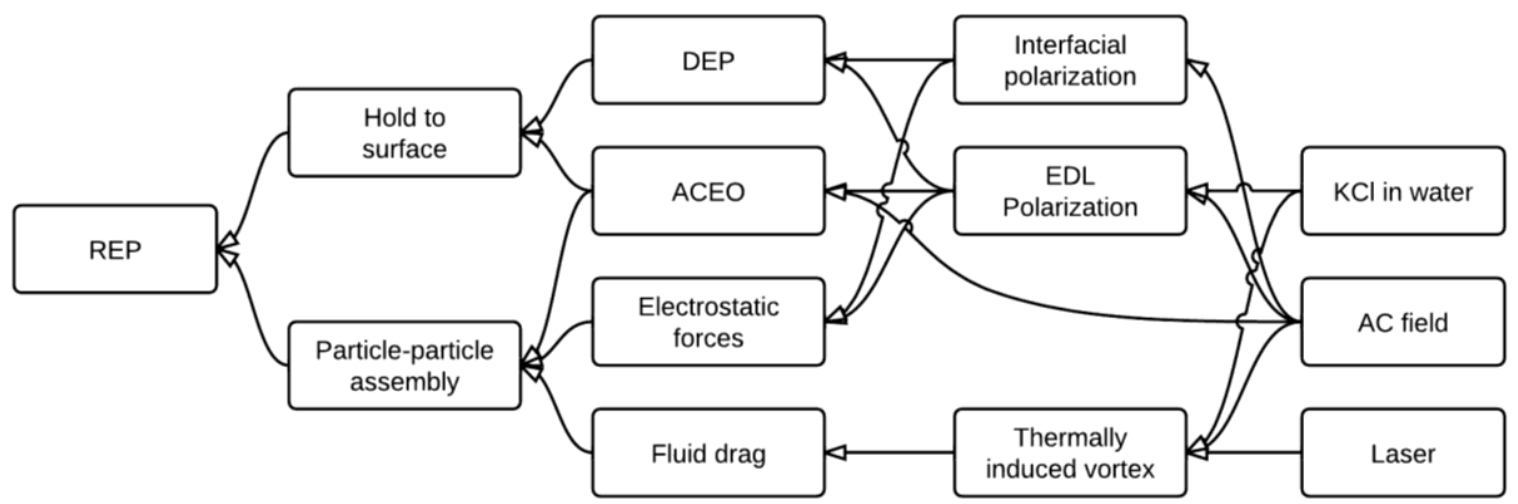

FIGURE 3 - The Relationship of the Mechanics of REP. 
The mechanisms involved in REP are interrelated. The AC field affects all of the different areas of REP, whereas the laser only affects the induced vortex. DEP and ACEO govern the particle-electrode holding force. Both polarization techniques are important to forces parallel and perpendicular to the field. Isolating the effects of one polarization mechanism is difficult as ACEO is thought to affect the spacing of the particles separately from the electrostatic forces and to induce seemingly random motion. In spite of the intertwined relationship between the various mechanisms involved in REP, control over the particles by changing the laser power, the AC field, and the conductivity of the medium via $\mathrm{KCl}$ concentration is straightforward. Varying any given individual parameter has a predictable and simple effect, as shown in Results and Discussion.

This thesis intends to characterize the crystalline nature of the aggregations of particles with the understanding of the underlying mechanics. The ability to identify the direct effects of a specific polarization mechanism was discussed, but is not demonstrated. Polarization identification was considered outside of the scope of the thesis, and is recommended for future work pertaining to electrokinetic spectroscopy. 


\section{MATERIALS AND METHODS}

\section{A. Devices}

The REP devices used were constructed using a glass slide and a cover slip both coated with indium tin oxide (ITO). A channel was manually cut into $50 \mu \mathrm{m}$ doublesided tape, which was used to connect the two glass slides with the ITO-surfaces facing each other. Holes were drilled in the glass slide to allow fluidic access to the channel, with adhesive rubber ports covering the holes. Lead wires were attached to the ITO surfaces using conductive epoxy, conductive copper tape, and solder. Figure 4 is an image of the device used for testing taped to a petri dish (with a hole cut for the objective).

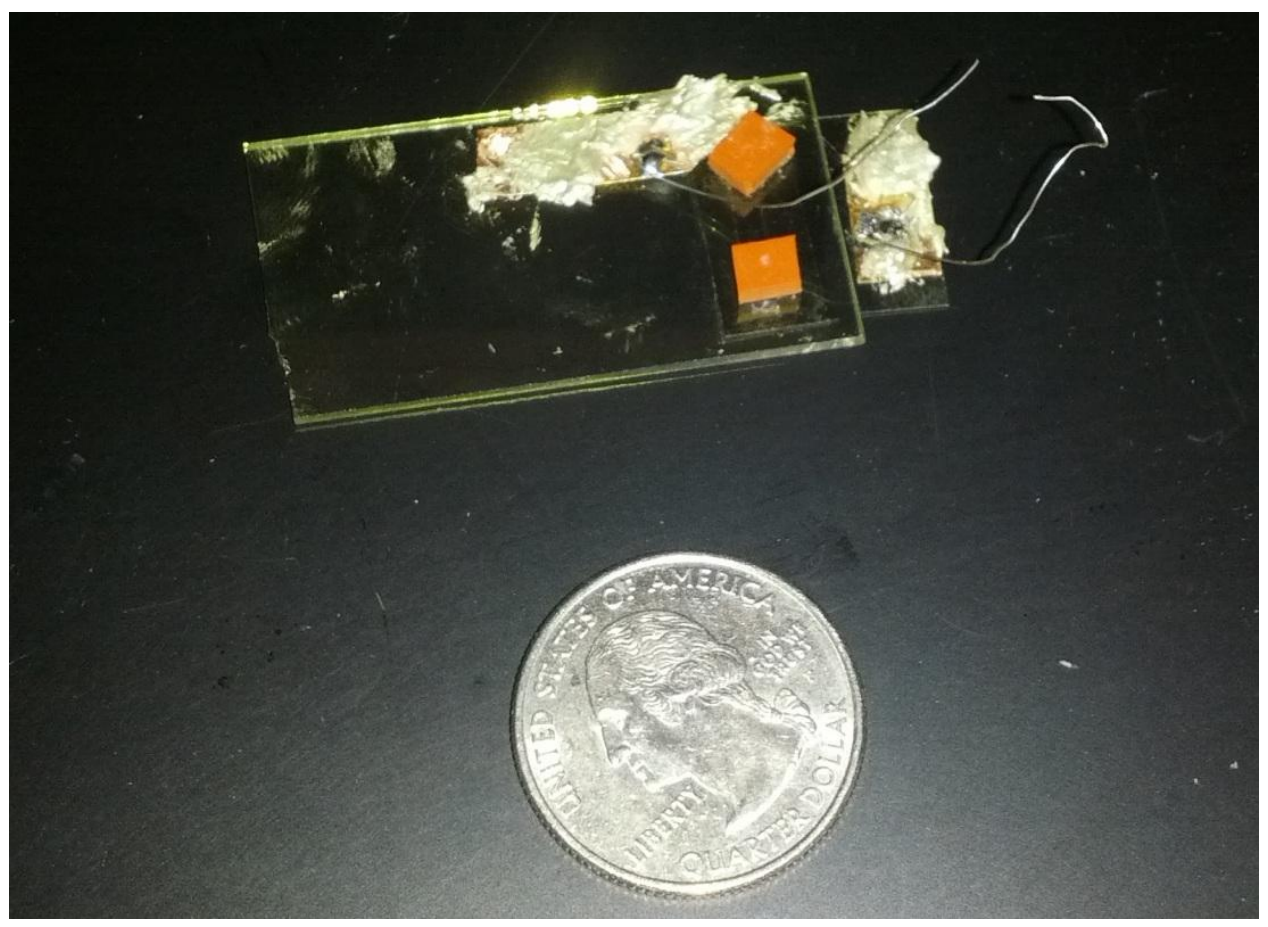

FIGURE 4 - REP device used for testing.

The device shown is taped to a petri dish with a hole in the center for access. The ports are drilled into the glass as it is more rigid than the cover slip. Adhesive rubber 
ports are used to reduce the effect of external air movement and evaporation, and to reduce the size of the opening to prevent the collection of unwanted material.

\section{B. Consumables}

Inserted into the device was an aqueous $\mathrm{KCl}$ solution containing $1 \mu \mathrm{m}$ polystyrene particles. The conductivity of the solution was $2.5 \mathrm{mS} / \mathrm{m}$. Thermo Scientific Fluoro-Max aqueous polystyrene particles were used, having a density of $1050 \mathrm{~kg} / \mathrm{m}^{3}$ (Scientific, 2012). The particles were red fluorescent; as green light hit the particles, they omitted red light.

\section{Laboratory Equipment}

A Nikon Eclipse $\mathrm{T} i$ inverted microscope was used with a 60x water-immersion objective. To capture images, a ThorLabs DCU223C camera was utilized, imaging 4.65 $\mu \mathrm{m}$ per pixel (square) at $30 \mathrm{~Hz}$ with a resolution of 1024 x 768 pixels. An X-Cite $120 \mathrm{Q}$ mercury lamp was used to provide green light to illuminate the particles. Two dichroic mirrors were used to allow infrared and green light to the sample, and only red light back into the camera. Figure 5 shows the experimental set up, and Figure 6 shows the Nikon Eclipse Ti used. 


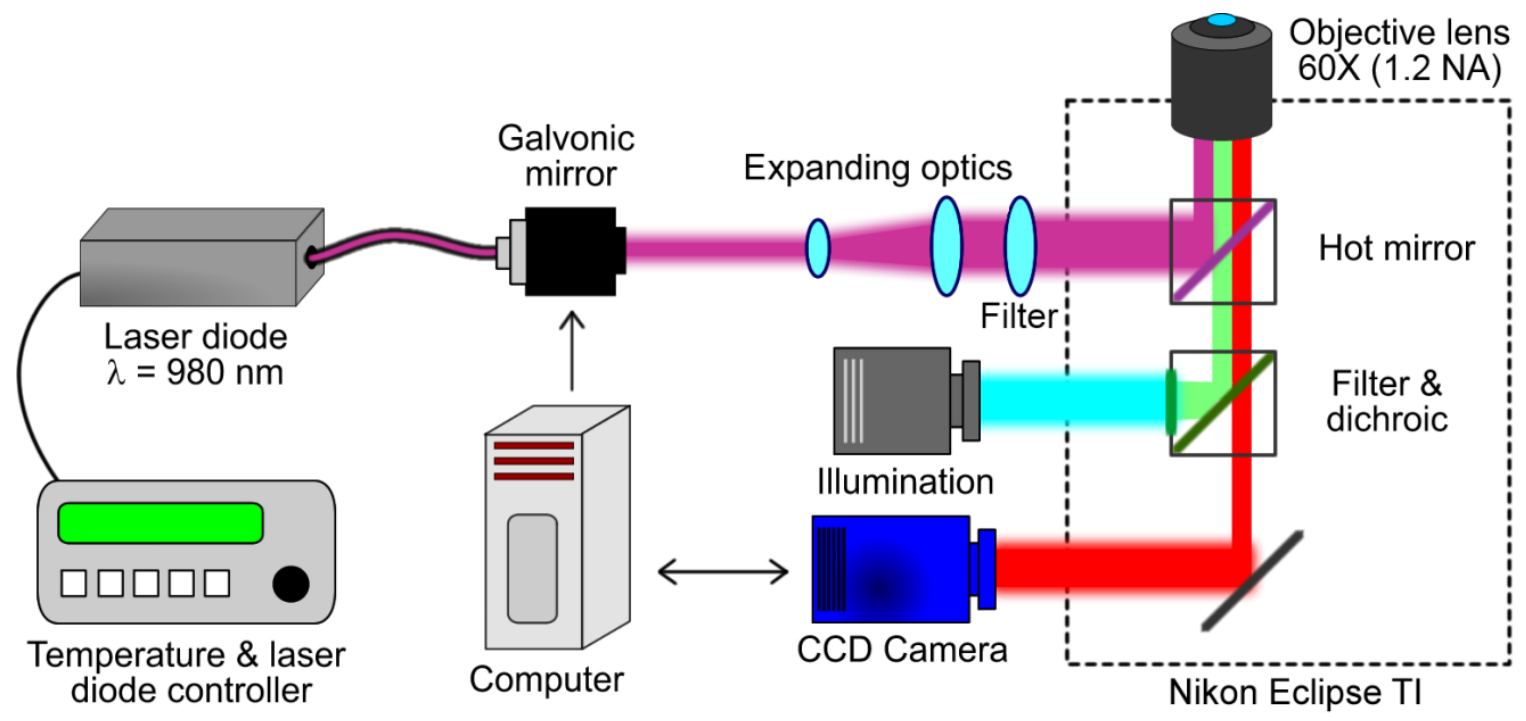

FIGURE 5 - Experimental REP set up, modified from (Velasco et al., 2012).

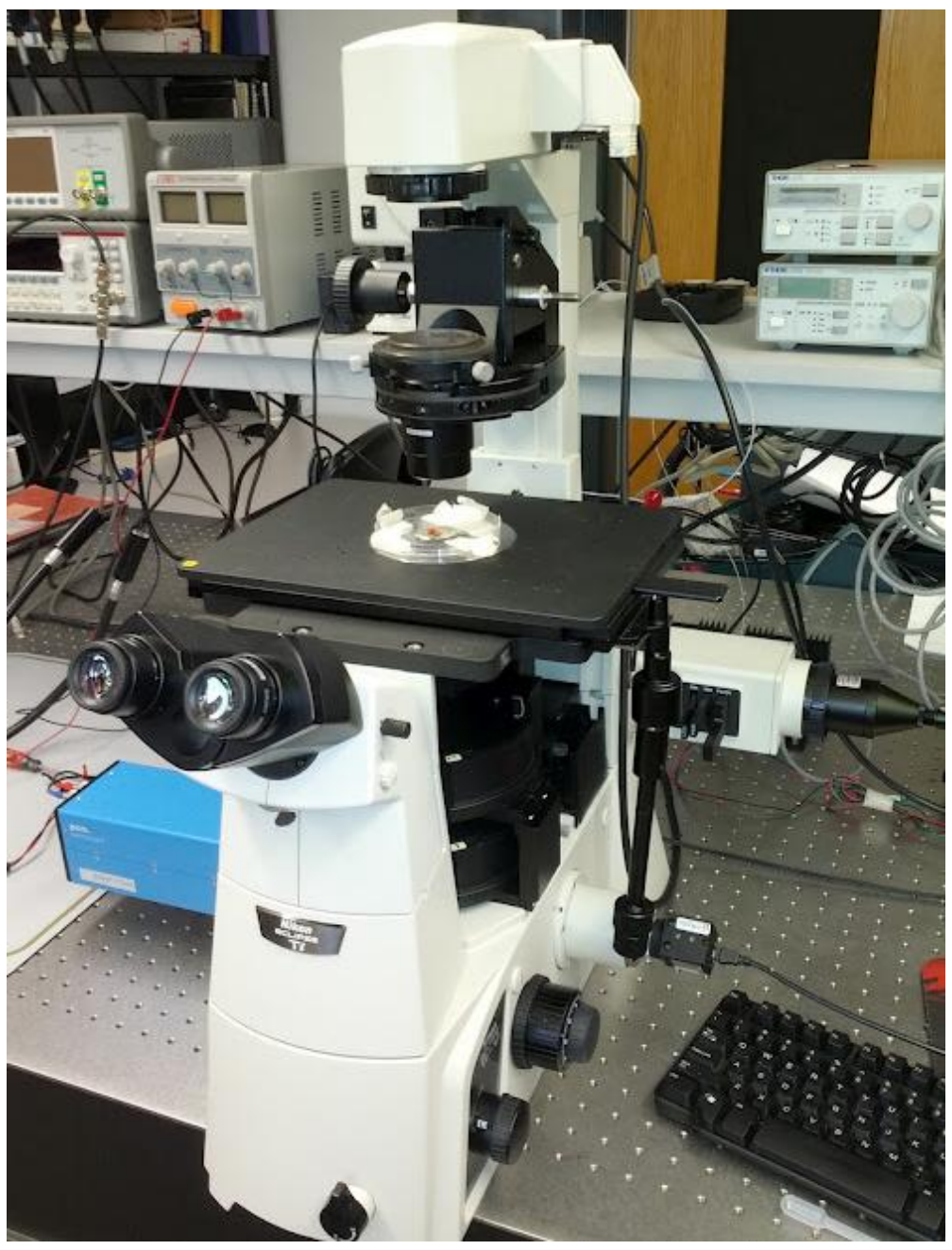

FIGURE 6 - Nikon Eclipse Ti microscope and other equipment. 
A ThorLabs PL980P330J butterfly infrared laser diode (975 nm) was used in conjunction with a ThorLabs TED200 temperature controller and a ThorLabs LDC220C current controller for heat generation. A ThorLabs NENIR05 absorptive filter was used to lower the laser power in some experiments order to raise the power to the diode. The diode had minimal output until the power input reached $\sim 70 \mathrm{~mW}$. Using a filter allowed experiments to be conducted at a higher input power where the characteristics of the diode were better defined.

A ThorLabs scanning galvo mirror system was used to control the location of the laser on the surface of the electrode. The laser was focused using the 60x microscope objective (1.2 NA), which was also used for viewing. The collimated laser was widened using two convex lenses with focal lengths of $40 \mathrm{~mm}$ and $150 \mathrm{~mm}$. A ThorLabs Scanning Galvo System PSU was used in conjunction with a custom LabVIEW program to control the position of the laser (see appendix). The LabVIEW program made the laser move through 1000 discrete points along a scanned line in one direction. The scan occurred in either continuous mode or unidirectional mode. In continuous mode, the laser would travel one direction down the scanned line, and at the end, change directions and return at the same speed. In unidirectional mode, the laser would travel one direction down the scanned line, then very quickly travel to the other end and start over. The scan frequency was varied by increasing the rate each data point was processed. As an example, a unidirectional scan at $10 \mathrm{~Hz}$ would scan the laser through 10000 points per second, while a continuous scan at $10 \mathrm{~Hz}$ would scan the laser through 20000 points per second. 
Images captured were then processed with several Matlab scripts (see appendix). The red image was converted to grayscale, and particle locations were identified assuming a Gaussian distribution of fluorescence by each particle. Figure 7a shows a cropped image before processing, Figure $7 \mathrm{~b}$ shows a grayscale image with the peaks identified overlaid by bright red circles.

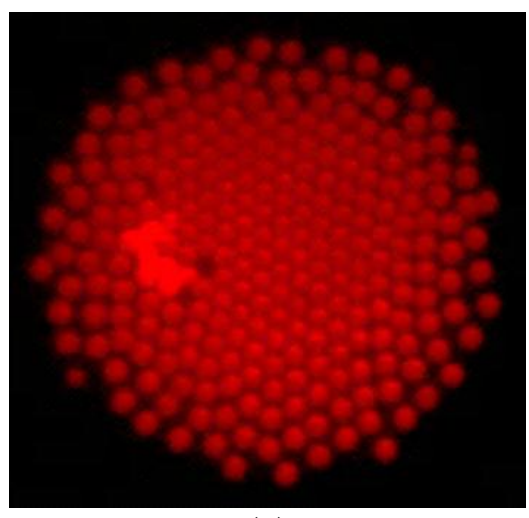

(a)

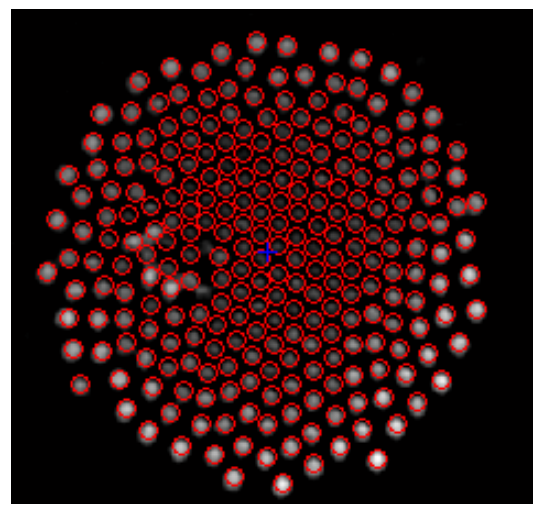

(b)

FIGURE 7 - (a) Unprocessed image of dot aggregation. (b) Thresholded, grayscale image with identified peaks circled. $35 \mathrm{kHz} \mathrm{AC}, 6.42 \mathrm{~V}, 24.3 \mathrm{~mW}$ laser power.

As shown in Figure $7 \mathrm{~b}$, several particles were not identified just left of the aggregation center. The same group of particles shown in Figure 7a appears brighter; this is due to a second layer of particles. This represents a problem in analyzing REP images: incorrect particle identification may result from images with a double layer of particles. This is made evident by some particles being identified too close to each other, below the minimum $1 \mu \mathrm{m}$ limit for $1 \mu \mathrm{m}$ particles. Also noticeable in Figure $7 \mathrm{a}$ is that some of the particles appear dim; care must be taken to insure they are not below the specified detection threshold. 


\section{Algorithms}

\section{Particle Count Reduction}

Once particles have been identified, it is determined whether or not each given particle was a captured particle. The distance of the particle from the center of the largest aggregation was utilized to remove certain particles from consideration, as well as particles with anomalously high spacing between their neighbors. Figure 8 shows located peaks (circles) and peaks retained for analysis (filled in circles).

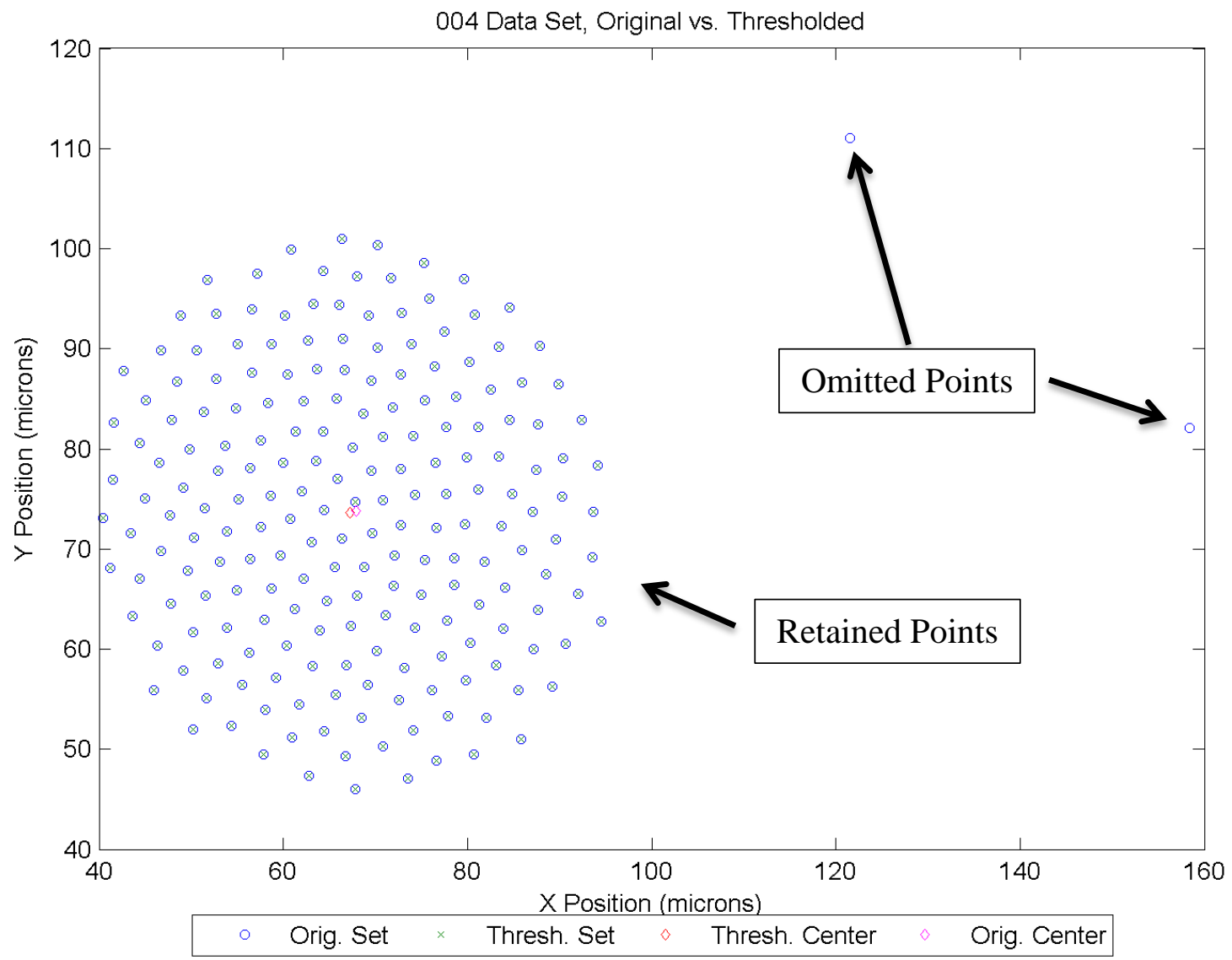

FIGURE 8 - Sample particle selection.

From Figure 8, the two particles far removed from the aggregation were excluded from the data set. These two particles were excluded due to having anomalously-high 
spacing, and also for being far outside of the radius of the aggregation. The center of all of the imaged particles is calculated, and the radial distance for each particle from that center is calculated. Each particle must then be under double the threshold of the average radial distance. The average spacing with the six nearest neighboring particles is calculated for each particle, and any particle with spacing above the average of all of the particles in the image multiplied by 1.25-2.0 was removed. The multiplier used for each threshold was adjusted for each set of images to minimize the number of lost particles (particles captured but ignored), while ignoring all non-captured particles. No one value worked for all image sets, and a range had to be used for analysis.

The aggregations forming due to a linear laser scanning pattern are treated differently. The using the average radius is not effective for thresholding points as it would tend to clip the ends of the line of aggregate particles, or not exclude particles a sufficient distance from the aggregation. As a result of this, each point was thresholded based only on its spacing.

\section{Crystallinity}

The crystallinity of an aggregation was determined using the average spacing of the particles between their neighbors. The average spacing used for determining crystallinity was calculated using the nearest three particles to each particle in the aggregation. This value was then averaged over the total particle population.

Only the three nearest particles were used for each particle to reduce the effect of particles near the aggregation edge. In a perfect crystalline grid, the edge particles will not have six neighbors, while an interior particle would. Using the nearest six particles 
for an edge particle would result in a higher spacing for that particle. An attempt at removing edge particles was used to little effect, and is discussed in Appendix II.

\section{Velocity and Acceleration Calculation}

Velocity was calculated using a fourth-order central differencing scheme given as (Bhat, 2004)

$$
u_{i}=\frac{-P_{i+2}+8 P_{i+1}-8 P_{i-1}+P_{i-2}}{12 h}
$$

where $P_{i}$ is the particle location at time-step $i$, and $h$ is the length of the time-step. Acceleration was calculated using (Bhat, 2004)

$$
v_{\mathrm{i}}=\frac{-\mathrm{P}_{\mathrm{i}+2}+16 \mathrm{P}_{\mathrm{i}+1}-30 \mathrm{P}_{\mathrm{i}}+16 \mathrm{P}_{\mathrm{i}-1}-\mathrm{P}_{\mathrm{i}-2}}{12 \mathrm{~h}^{2}}
$$

Correspondingly, five images were used to calculate both velocity and acceleration to provide reasonable estimates. Providing an estimate of the acceleration was used to demonstrate that the particles reach terminal velocity, as discussed in section I, subsection C3.

\section{E. Parameters}

Several important parameters were selected for characterization: the length of the laser scan, the power of the laser, the frequency of the applied AC field, the voltage of the applied AC field, and the scanning frequency of the laser. Outside of the scope of this work were other parameters, including the size of the particles used, the number of particles in each aggregation, and the conductivity of the medium. The size of the particles and the conductivity were held constant. The number of particles in an aggregation is a function of the tested parameters, and the concentration of suspended particles. 


\section{Results and Discussion}

\section{A. Particle Spacing by Radius}

The particle spacing in aggregations showed interesting characteristics. The spacing towards the edges tended to be higher than the spacing in the center of aggregations, as expected. Spacing also seemed to be affected by some unknown factors, perhaps scratches on the electrode surface. The particle-particle spacing for an aggregation, produced by a static laser, is shown in Figure 9.

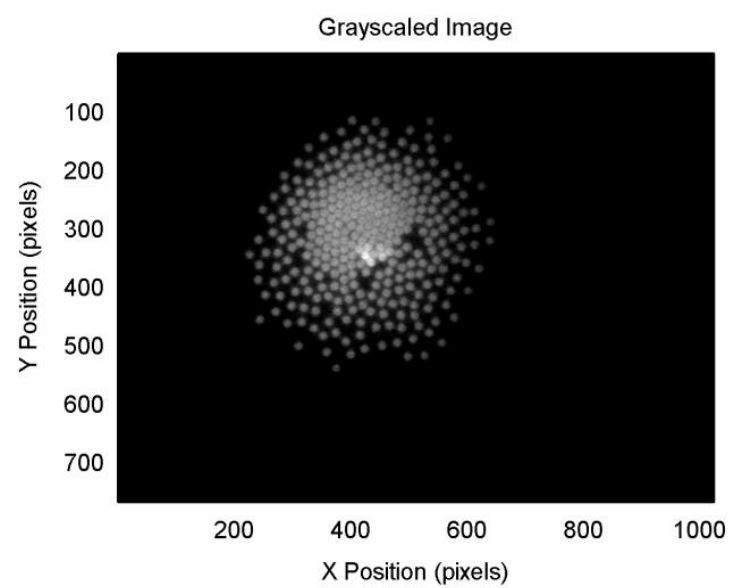

(a)

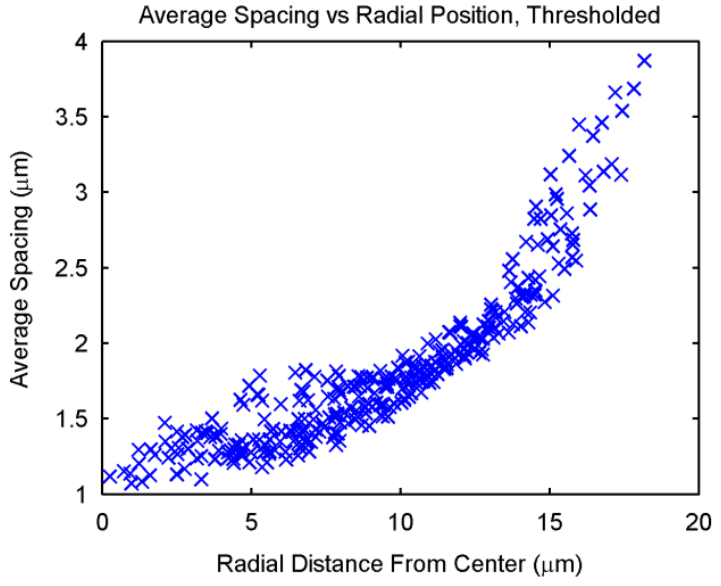

(b)

FIGURE 9 - Particle spacing versus radial position in a dot aggregation. (a \& b) $30 \mathrm{kHz}$ $\mathrm{AC}, 6.533 \mathrm{~V}, 11.85 \mathrm{~mW}$ laser power.

The spacing forms a characteristic shape such that the particle-particle spacing slowly increases until it reaches a certain point at which the spacing increases more rapidly. This characteristic was present in all tests; the particle spacing would dramatically increase towards the edges of the aggregation. This is demonstrated more easily with dot aggregations (such as in Figure 7a) as line-scanned aggregations are not axisymmetric. Additional results are shown in Appendix III. 
The cause for this characteristic shape may be fluid drag. The outer particles experience the most drag, but share that drag force with the particles on the inside of the aggregation. A particle close to the edge, but not on the outside, will experience drag pushing it towards the center of the aggregation, in addition to forces from the outside particles pushing it towards the center. Particles close to the center of the aggregation will experience little, if any, direct hydrodynamic drag. As the spacing in the aggregation minimizes, the ability of fluid drag to affect the interior particles diminishes.

\section{B. Patterning}

Of primary interest is the ability to pattern crystalline structures. Examined herein are dot- and line-shaped aggregations. These structures are important as they are the two must fundamental shapes that can be formed, and if thoroughly understood, could be used to construct more complex shapes. Two sample line aggregations are shown in Figure 10.

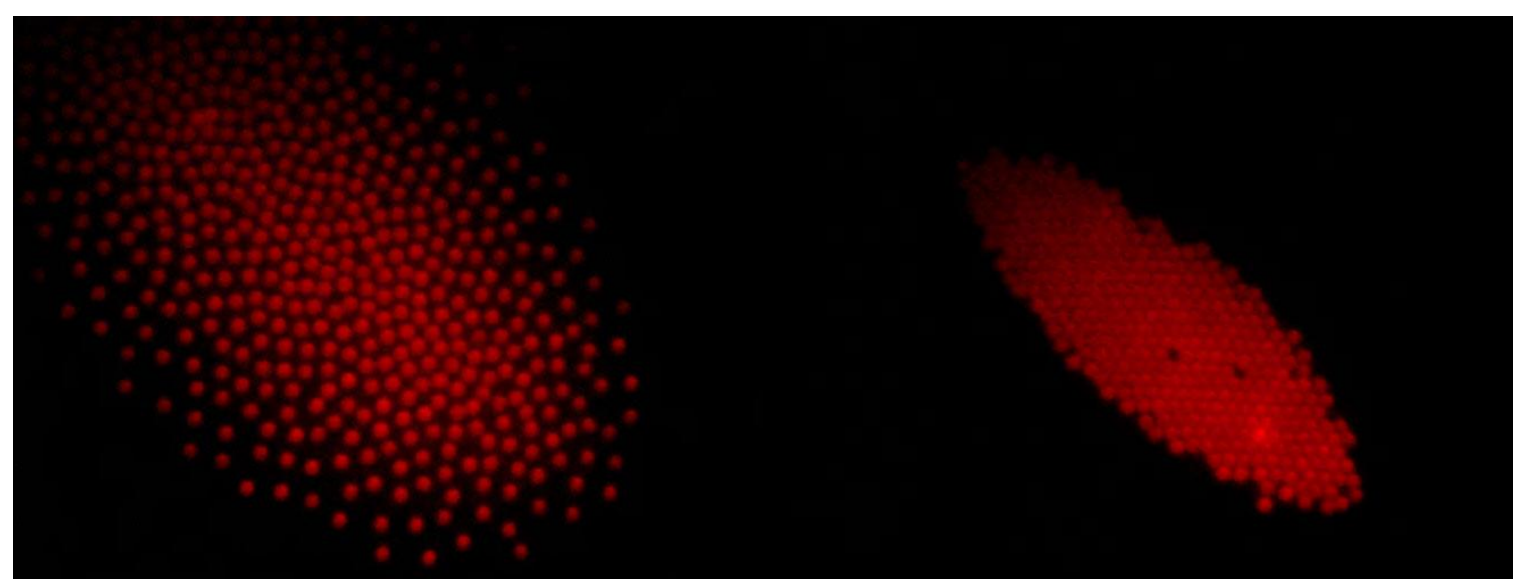

(a)

(b)

FIGURE 10 - Comparison of scanned line aggregations. (a) $25 \mathrm{kHz} \mathrm{AC}$, (b) $70 \mathrm{kHz} \mathrm{AC}$, (a \& b) $6.291 \mathrm{~V}, 24.3 \mathrm{~mW}$ laser power, $16 \mathrm{~Hz}$ continuous scan, $53.4 \mu \mathrm{m}$ scan length.

The $70 \mathrm{kHz}$ sample is more crystalline, even towards the edges. The $25 \mathrm{kHz}$ sample, however, has a much higher spacing, and is not crystalline in form. The average 
spacing in the $70 \mathrm{kHz}$ sample is $1.12 \mu \mathrm{m}$, whereas the average spacing in a $25 \mathrm{kHz}$ sample is $1.75 \mu \mathrm{m}$. Both samples were generated by scanning the laser continuously back and forth at $16 \mathrm{~Hz}$. The change in particle spacing from the edge to the center of the aggregation is very apparent in Figure 11 (a), but much less obvious in Figure 11 (b). As the frequency is increased, the electric force between the particles decreases.

In order to characterize the ability to pattern a line-aggregation, five fundamental control parameters are individually characterized: AC frequency, laser scan frequency, laser power, laser scan length, and AC voltage. Each characterization figure displayed shows the average particle-particle (center to center) spacing with an ' $\mathrm{X}$ ', the corresponding number of particles with an 'O', and two sets of standard deviations shown with error bars. Each data point was imaged 31 to 64 times at a frame rate of $30 \mathrm{~Hz}$. The number of particles is the average number of particles identified within each image set.

The larger set of error bars corresponds to the standard deviation of the particleparticle spacing per image, averaged over the image set. The smaller set of error bars corresponds to the standard deviation of the average particle-particle spacing per image (or the standard deviation of the image set's average spacing). The larger set of error bars is larger due to the large range of spacing in each image of the image set. The smaller set of error bars is centered around the average spacing data point, and is \pm 1 standard deviation from the data point (making it two standard deviations in length). The larger set of error bars is also two standard deviations in length, but is not centered about the data point. The center of the larger set of error bars was shifted such that the upper portion of the error bars was proportional to the difference between the average maximum spacing in the image set and the average spacing, while the lower portion was 
proportioned to the difference between the average minimum spacing in the image set and the average spacing. This demonstrates that the particles deviate less below the average spacing than they do above the average spacing.

The larger set of error bars was higher than measured values. The Matlab script used to locate the particles had a degree of randomness in identifying the particle's location. The Matlab script assumed a Gaussian distribution of light from each individual particle; however, images of the particles demonstrated that the peak brightness wasn't necessarily at the center of a particle. This degree of randomness contributed to the standard deviation of each aggregation, particularly more compact aggregations.

\section{AC Frequency Characterization}

The polarization of the particles was frequency dependent, and since polarization mechanisms will relax at higher frequencies, adjusting the frequency provided a large range in particle-particle spacing. Figure 11 shows the average particle spacing as a function of the AC Frequency. 


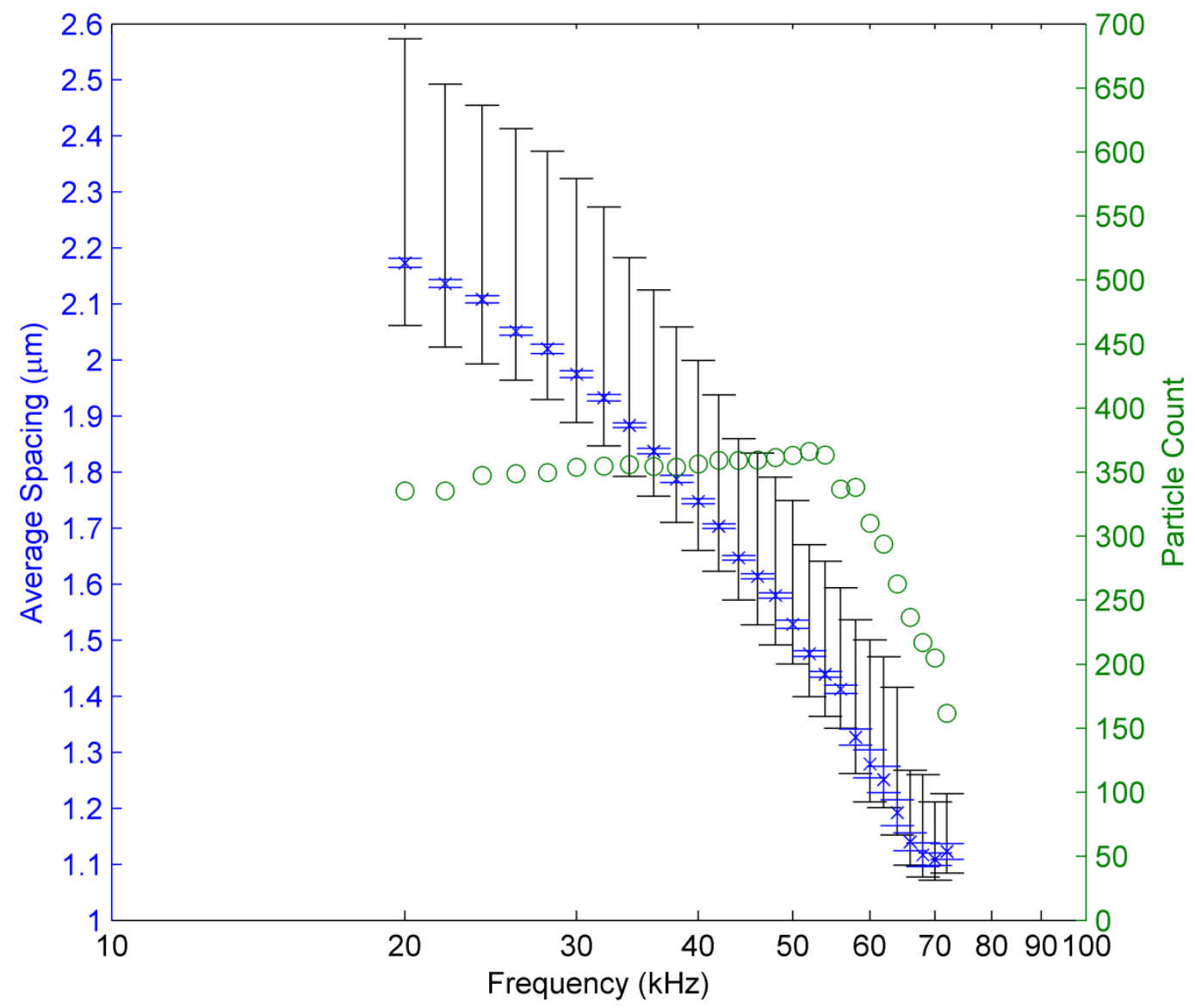

FIGURE 11 - Average spacing versus AC frequency. 6.535-6.563 V, $11.85 \mathrm{~mW}$ laser power, $16 \mathrm{~Hz}$ continuous scan, $59.3 \mu \mathrm{m}$ scan length.

As expected from Figure 10, the spacing decreased with increasing frequency. This is due to the relaxation of one of the particle polarization mechanisms. There is a very slight transition in the slope of the data points near $25 \mathrm{kHz}$, and it is possible that this is where one polarization mechanism relaxes and the other mechanism continues, but that is merely speculation without additional data at lower frequencies. Regardless of which mechanism is dominant, the polar moment decreases and the electrostatic forces between the particles decrease. This phenomenon is reflected in the change in Figure 10 from (a) to (b). The characteristic transition in particle spacing with respect to radial 
position is more obvious in Figure 10a, but is still present in (b); it has just moved nearly to the edge (notice the outermost ring of particles has varied spacing).

Another interesting characteristic of Figure 11 is the data between 66 and $72 \mathrm{kHz}$ where the average spacing is more constant. Whether or not the particles reached a true minimum is difficult to tell; data could not be acquired at higher frequencies without the particles being released from the electrode surface. Also, the number of particles trapped in the aggregation drop sharply at $54 \mathrm{kHz}$. At $74 \mathrm{kHz}$, particles would no longer remain on the surface. AC Frequency tests were run in consecutive order, from $20 \mathrm{kHz}$ to 72 $\mathrm{kHz}$, with the same aggregation of particles. This was important to insure the solution changed as little as possible for this group of tests, and because higher frequencies tend to remove particles from the surface (note the diminishing particle count). To insure data was calculated properly, the spacing results were spot checked at higher frequencies. A sample spot check is presented in Figure 12.

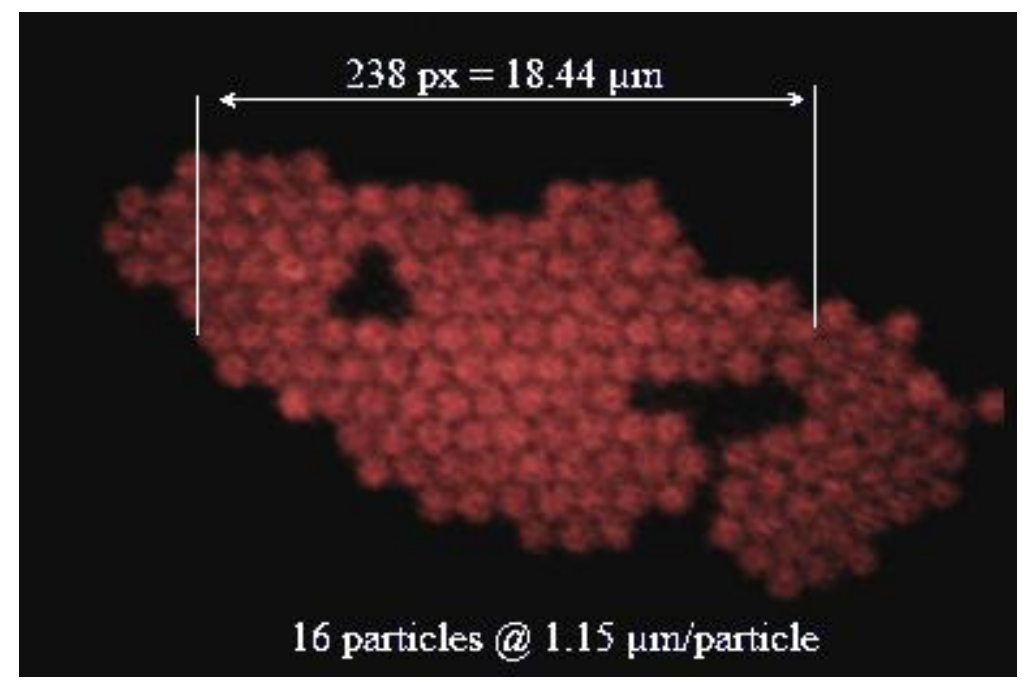

FIGURE 12 - Alternate spacing calculation spot check sample. $72 \mathrm{kHz}$ AC, $6.563 \mathrm{~V}$, $11.85 \mathrm{~mW}$ laser power, $16 \mathrm{~Hz}$ continuous scan, $59.3 \mu \mathrm{m}$ scan length.

A sample image was taken from the $72 \mathrm{kHz}$ data, and rotated such that the particles would align with the x-axis. The number of pixels was counted and converted 
into microns. As shown in Figure 12, particle spacing was above the $1.12 \mu \mathrm{m} /$ particle shown in Figure 11. This was repeated for three rows in different directions with similar results. This may indicate that the Matlab script use was yielding slightly lower-than-real spacing, but the difference was within $3 \%$. Such analysis was only possible when the particles formed a nearly uniform crystal (high frequencies), and neglects the gaps in the aggregation and increased spacing from edge particles.

The spot check presented in Figure 12 and the data presented in Figure 11 indicate that the particles, when tightly packed, do not touch (on average). In order to maintain a non-zero space between particles, strong electric repulsive forces must exist between the particles. It is evident that the rate of change of particle spacing by frequency abruptly changed when the particles became close, and is therefore hypothesized that a polarization mechanism relaxed. If this is the case, it is most likely that the MaxwellWagner interfacial polarization relaxed. DLVO theory for the force between two particles with ionic double layer polarization models the force as a function of $\mathrm{e}^{-\mathrm{kd}}$, which, due to the inverse Debye length being very large $\left(10^{9} \mathrm{~m}^{-1}\right)$, decays much faster than the published model for interfacial polarization. A force so highly dependent on spacing would explain an abrupt stop in the contraction of the aggregation while maintaining highly uniform spacing from center to edge.

The average standard deviation of particle spacing (black bars) shows that the spread of the particles significantly decreases with increasing frequency. This shows that as an aggregation of particles contracts, the spacing becomes more uniform throughout the aggregation. This is reflected in Figure 12. In a compact aggregation, a small standard deviation indicates that it is more uniformly crystal, such as in Figure 10b. A 
higher standard deviation indicates that the spacing varies much more significantly from the center of an aggregation to the edge, such as in Figure 10a.

\section{Laser Scanning Frequency Characterization}

The next parameter characterized was the scanning frequency of the laser. The scanning frequency of the laser controls the uniform nature of the vortex created, and the transition from dragging an aggregation to spreading the aggregation into a line. Figure 13 shows the average spacing for an aggregation versus the laser scanning frequency.

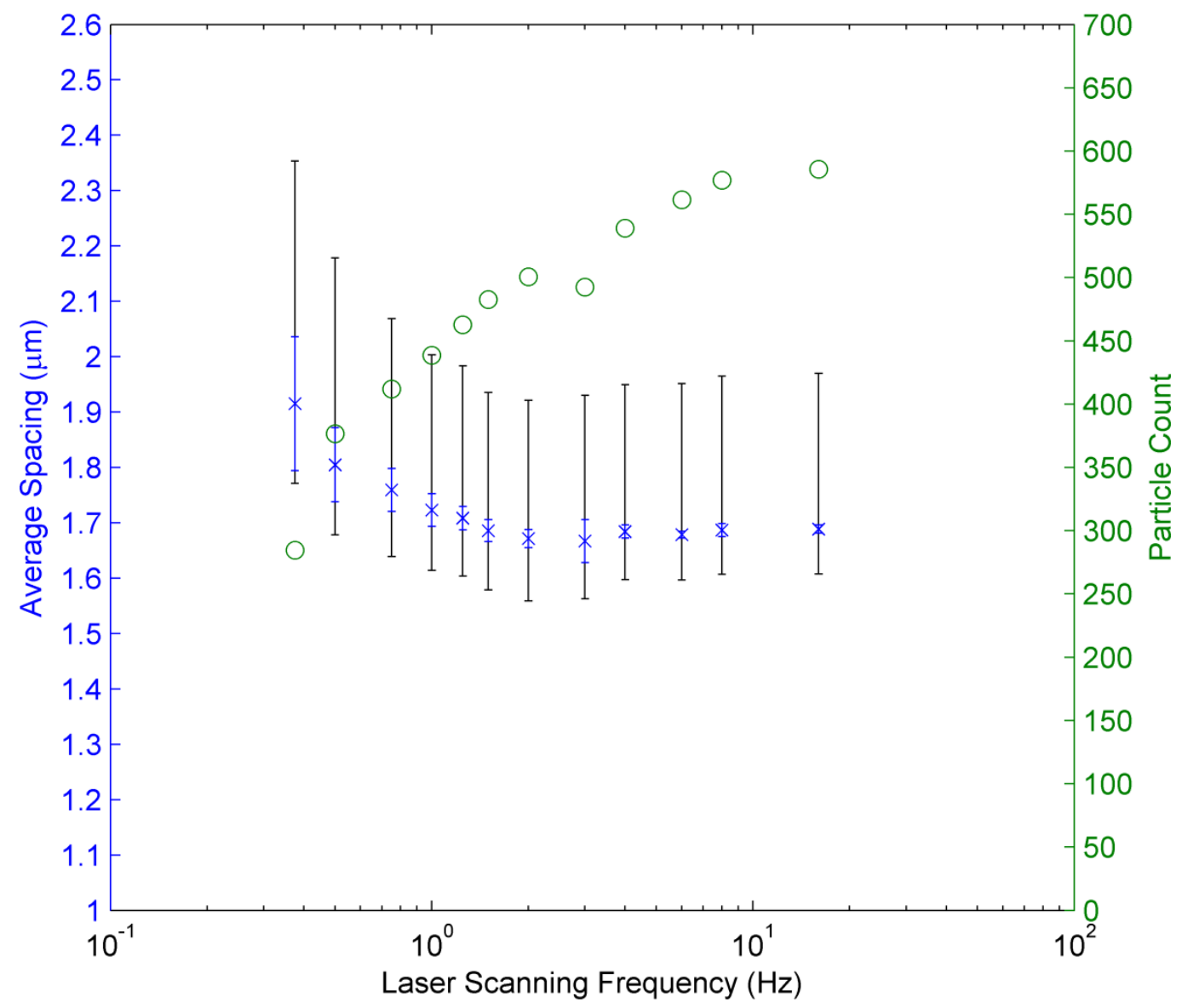

FIGURE 13 - Average spacing versus laser scanning frequency. (6.34-6.36 V, $30 \mathrm{kHz}$ $\mathrm{AC}$, continuous scan, $24.3 \mathrm{~mW}$ laser power, $53.4 \mu \mathrm{m}$ scan length) 
As Figure 13 shows, larger scanning speeds decrease the aggregation spacing, to a point. This may be explained, in part, by the increase in the number of particles in the aggregation. More important is the tendency for the average aggregation spacing to converge. As time elapses and frequency increases, more particles enter the aggregation. The convergence of spacing is less affected by the number of the particles than the frequency. A large number of particles provided a range in spacing from the center of the aggregation to the edge, similar to Figure 9. A higher scanning frequency makes the thermal gradient converge to a mean value across the scan length, which means that the particles will not be dragged in one direction or the other. At low frequencies, particles will move with the laser. Figure 14 shows an aggregation scanned at $0.375 \mathrm{~Hz}$.

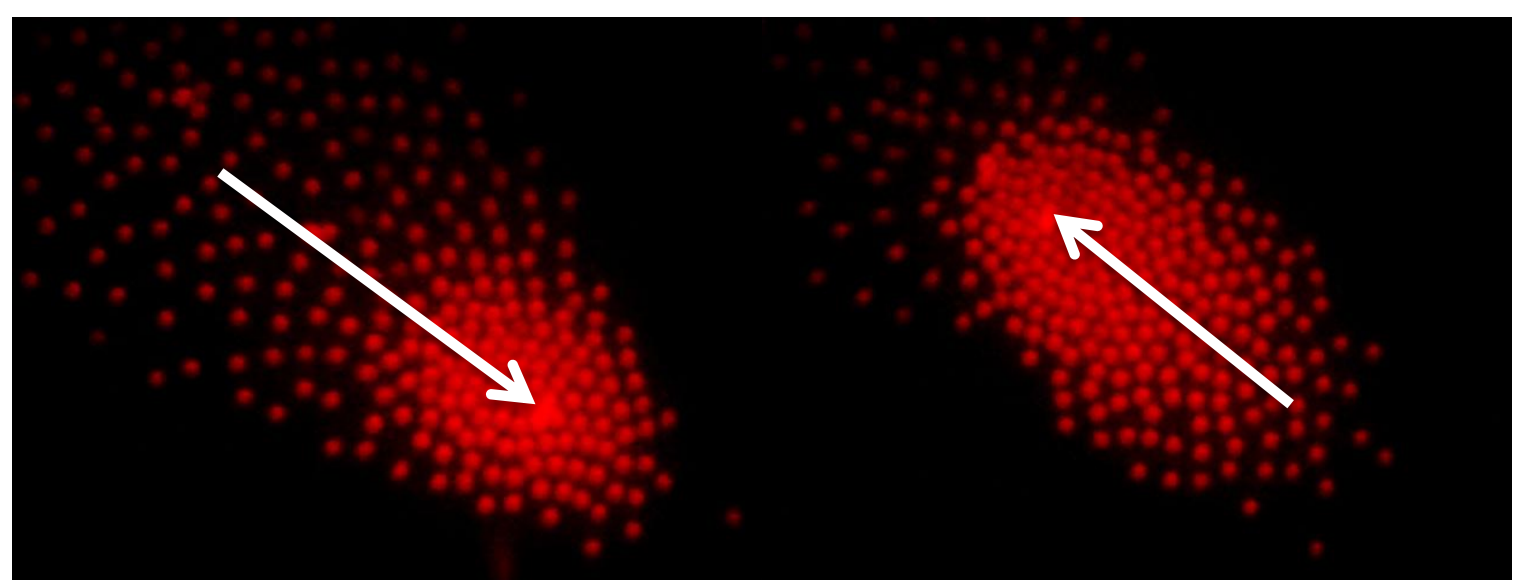

(a)

(b)

FIGURE 14 - Slow scanning speed time step, arrow indicating path of laser. $30 \mathrm{kHz} \mathrm{AC}$, $6.358 \mathrm{~V}, 24.3 \mathrm{~mW}$ laser power, $0.375 \mathrm{~Hz}$ continuous scan, $53.4 \mu \mathrm{m}$ scan length, $0.33 \mathrm{~s}$ lapse.

As shown in Figure 14, at a low scanning speed, the aggregation is partially dragged along with the laser. This scan rate represents a transition in moving a dot and scanning a line. If the laser scanning speed is increased, the particles will tend to stop following the laser and form a static aggregation, such as in Figure 10. This transition is 
marked in Figure 13 by the high standard deviation of average particle spacing (smaller error bar set) at lower frequencies.

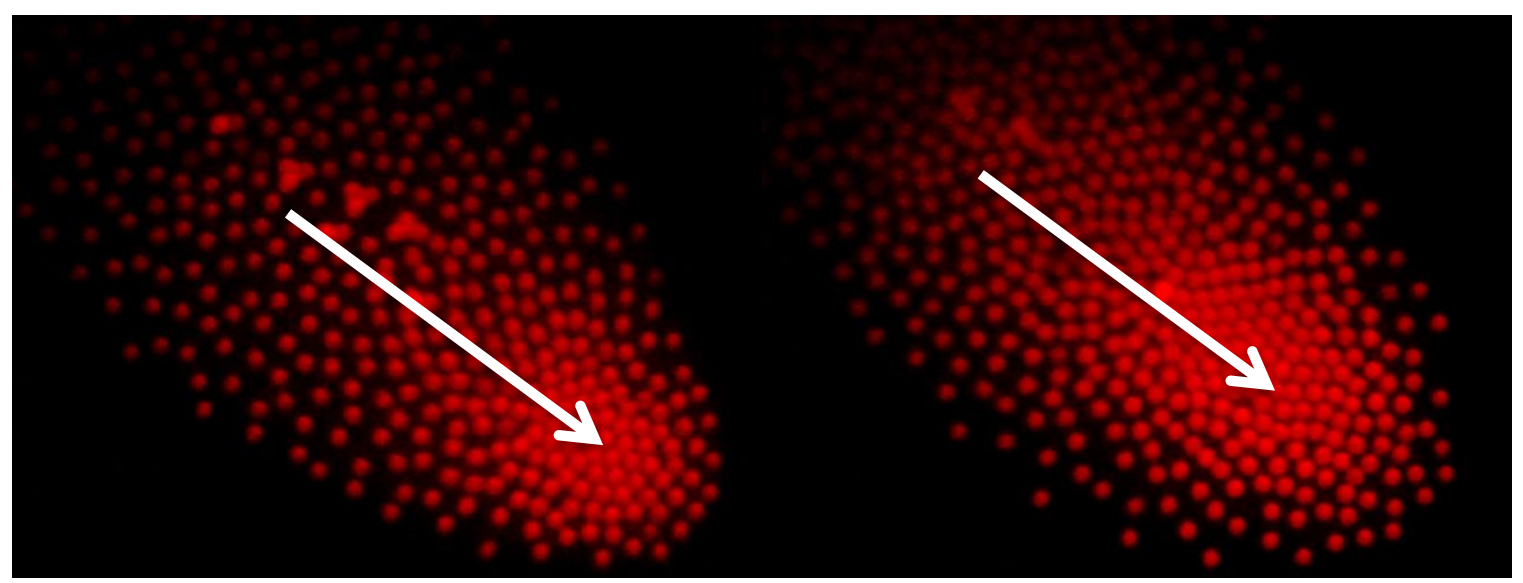

(a)

(b)

FIGURE 15 - Two different, low scanning speeds. $30 \mathrm{kHz}$ AC, $6.358 \mathrm{~V}, 24.3 \mathrm{~mW}$ laser power, $53.4 \mu \mathrm{m}$ scan length; a: $0.75 \mathrm{~Hz}$ cont. scan; b: $2 \mathrm{~Hz}$ cont. scan.

Figure 15 shows two more steps in the transition from a dot to a continuous line aggregation. As shown in Figure 13, the standard deviation for higher scan frequencies (including those shown in Figure 15) decreases; Figure 15shows that the $2 \mathrm{~Hz}$ scan has a larger compact group of particles than the $0.75 \mathrm{~Hz}$ scan.

Also shown in Figure 15a are multiple four-particle tetrahedron clusters. The worst case scenario for this having affected data is shown in Figure 15, and has statistically insignificant effects on processing. These particles could be joined together by ACEO or dipole-dipole attractive forces, but remains unexplored. Tests were run concurrently for the scanning frequency, meaning the $2 \mathrm{~Hz}$ scan was conducted after the $0.75 \mathrm{~Hz}$ scan, etc., on the same group of particles. 


\section{Laser Power Characterization}

The next parameter varied was the laser power. Tests on the laser power were run in consecutive order, similar to the AC frequency and scanning frequency tests. Figure 16 shows the spacing of an aggregation as a function of laser power.

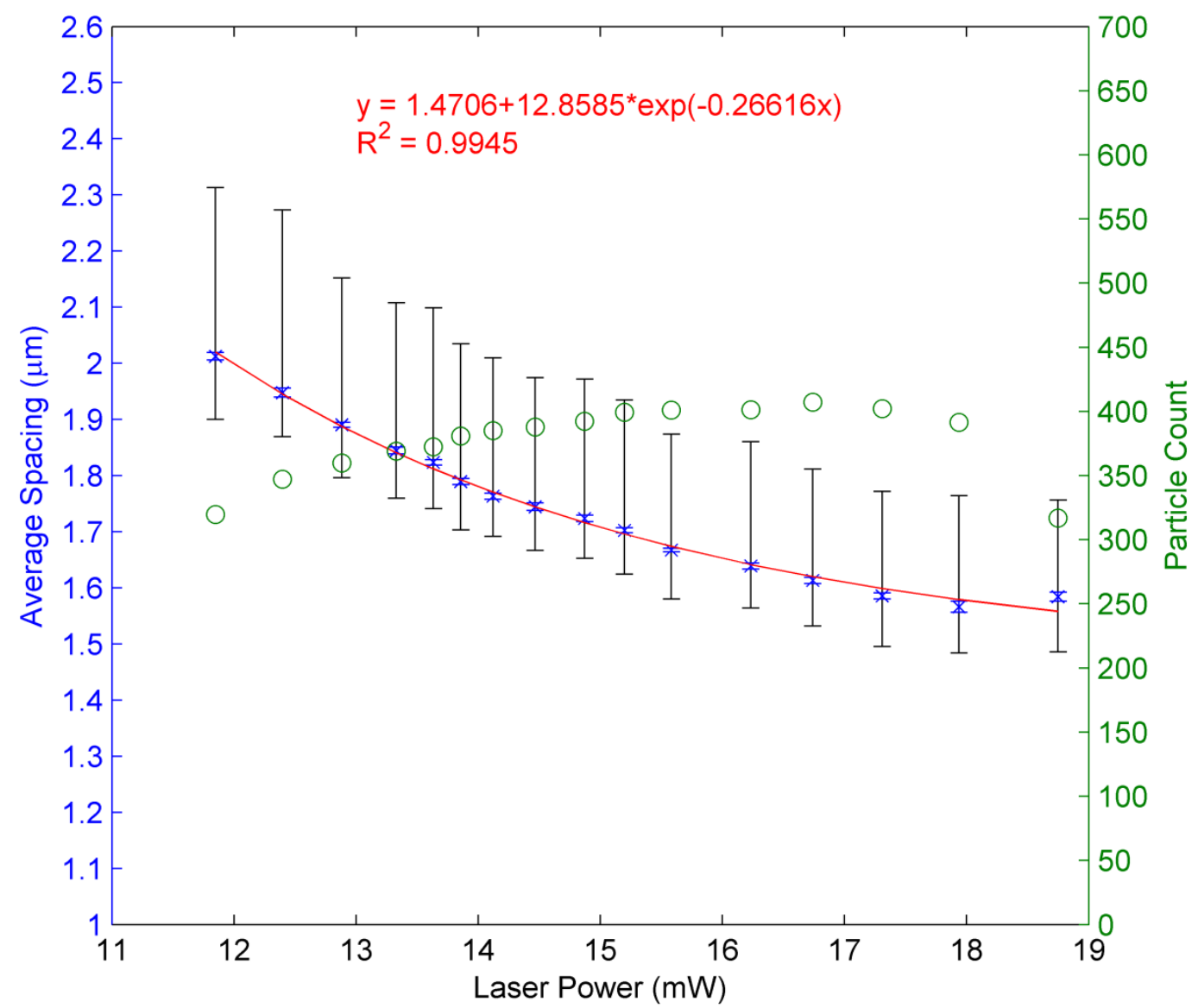

FIGURE 16 - Average spacing versus laser power with exponential fit. 6.55-6.57 V, 30 $\mathrm{kHz} \mathrm{AC}, 16 \mathrm{~Hz}$ continuous, $59.3 \mu \mathrm{m}$ scan length.

As shown in Figure 16, as the laser power increases, particle spacing decreases. The spacing should decay exponentially with increasing electrical forces, as the electrical forces should increase with decreasing distance proportionally to $\mathrm{d}^{-4}$ or $\mathrm{e}^{-\mathrm{d}}$. As shown in the figure, the data does fit an exponential pattern. The force exerted on the fluid (see 
eqn. 10) is linearly proportional to the thermal gradient, but the thermal gradient follows a Gaussian distribution (Velasco et al., 2012) and the change in fluid velocity was not necessarily linearly proportional to laser power. As such, the relationship between laser power and average spacing in an aggregation is not obvious.

The laser power affords an extra degree of control over an aggregation, but it was also more difficult to control. As the laser power increases, drag increases, but lift on the particles also increases. This effect tends to remove particles from the surface of the electrode, shrinking the aggregation size at higher laser powers. At lower laser powers, this mechanism is useful as an easy method to control the aggregation spacing dynamically, as can the AC frequency of the applied field.

Another explanation as to why the aggregation exhibits exponential decay is that the size of the aggregation consistently increased until the last several data points. On the last point, the particle count experienced a steep drop due to the vortex lift forces ripping particles away from the surface. The average spacing actually increased, showing that the spacing did affect the data. This by no means accounts for the total decrease in particle spacing over the range of laser powers, but may be responsible for its shape.

\section{Laser Scanning Length Characterization}

Scanning length is primarily of interest when trying to define the geometry of an aggregation. However, the length of scanning does have an effect on the average spacing of the aggregation. Larger spacing reduces the amount of time the laser spends at a given location, lowering localized temperature gradients, and increasing the effect of scanning speed on the difference between the local and mean thermal gradient as a function of time (the frequency became irrelevant as the scan length approached zero). This periodic 
effect on the local temperature was not desired; therefore a high scanning speed was used for these tests $(16 \mathrm{~Hz})$. Figure 17 shows the average spacing of an aggregation as a function of the laser scanning length.

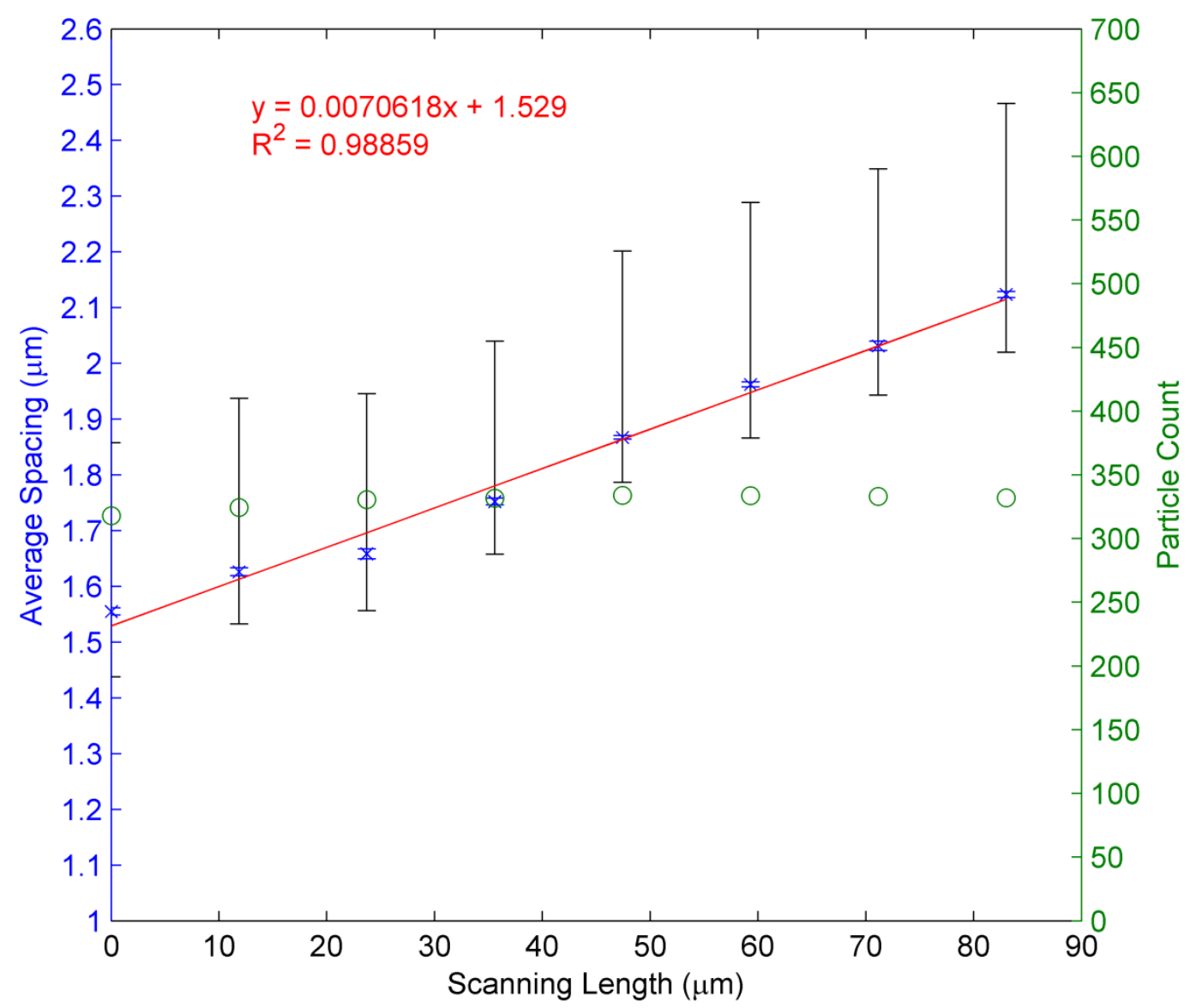

FIGURE 17 - Average spacing versus laser scanning length with linear fit. $6.35 \mathrm{~V}, 30$ $\mathrm{kHz} \mathrm{AC}, 11.85 \mathrm{~mW}$ laser power, $16 \mathrm{~Hz}$ continuous scan.

The scanning rate was set at $16 \mathrm{~Hz}$ to reduce periodic effects, as shown by the low standard deviation of the $16 \mathrm{~Hz}$ point in Figure 15. As expected, the spacing increases with increasing scan length. As the scan length is lengthened, heat is dissipated over a larger area. This effect seemed to be fairly linear, as demonstrated by the fit in Figure 17. The heat absorbed at any given point on the electrode surface is proportional to the cyclic 
area of illumination, and should be proportional to the scanning length when the scanning length is relatively large compared to the area of illumination when the laser is static. If not due to the small size of the range covered, the near linear nature of the data collected suggests that the exponential decrease in electrical forces as the spacing increased was counteracted. This effect could possibly be the shape of the aggregation, or perhaps the size of the aggregation. It may also have been that the drag force on the particles was related to the effective area of the vortex, and not the area hit by the laser; however, more information regarding the scanning laser electrothermal vortex is needed. Alternatively, larger scan lengths may be required to exhibit noticeable exponential behavior. The length of the scan was limited by the viewing area of the camera used, and a longer line could have yielded results more similar to those exhibited in Figure 16. The data obtained for average spacing versus laser scanning length was also acquired in sequential order.

\section{AC Voltage Characterization}

The final parameter investigated was the AC voltage. The voltage affects both the force creating the vortex and the dipole moment induced on the particles. Figure 18 shows the average spacing as a function of rms AC voltage. 


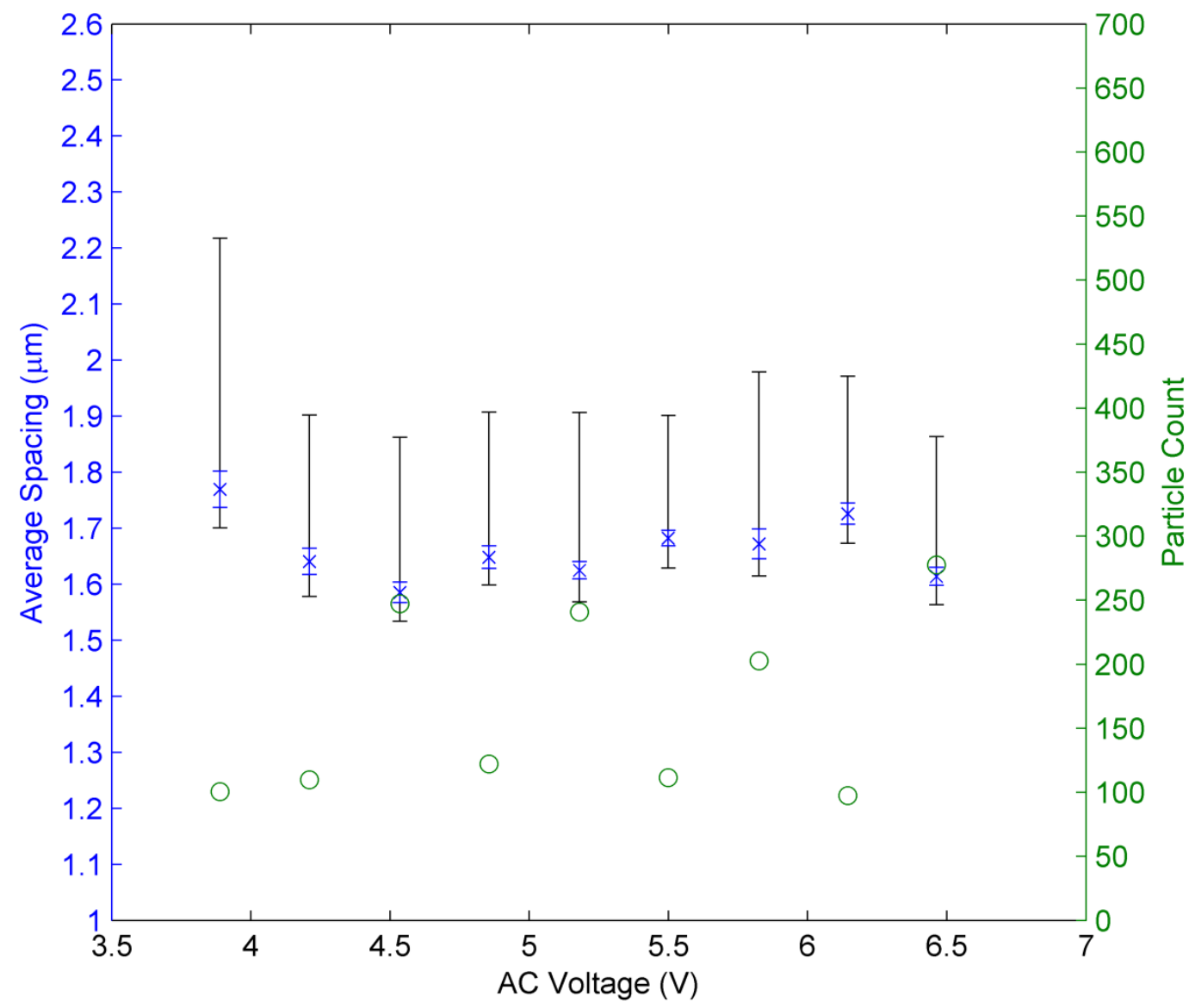

FIGURE 18 - Average spacing versus RMS AC voltage. $60 \mathrm{kHz}$ AC, $26.6 \mathrm{~mW}$ laser power, $30 \mathrm{~Hz}$ unidirectional, $77.1 \mu \mathrm{m}$ scan length.

AC voltage data was not acquired sequentially. The data points corresponding to a higher particle count were collected first, going from high voltages to low voltages. The lowest voltage point $(3.89 \mathrm{~V})$ was captured, and then the remaining low particle count points were captured from highest voltage to lowest (two high-to-low scans were run consecutively). AC voltage appears to not affect the average spacing strongly. This is because both the force creating the vortex (see eqn. 10) and the electrostatic force between the particles (see eqn. 6) are proportional to the square of the AC voltage. The 
seeming lack of effect on spacing due to voltage may indicate that the drag exerted on the particles is directly proportional to the force creating the vortex.

Also interesting was that the smaller aggregations tended to have a higher spacing. This suggests that the outermost particles in a larger aggregation experience more drag, and a higher fluid velocity. An important facet of future testing will be to characterize the crystallinity of an aggregation with respect to the size of the aggregation. Effects between aggregation sizes of 100 and 300 are noticeable, but the effect on data presented is assumed to be minimal. The sharp loss in the number of particles in the aggregation was due to the low frequency. A sequential test would have maintained a consistent particle count, as (mostly) demonstrated in Figures 12-18.

\section{Force Analysis}

\section{Velocity Analysis}

Force analysis was only performed on static laser aggregations due to their simplicity. Complete REP spectroscopy was not completed, but this section lays foundation for future work in this area, utilizing both dot and line shaped aggregations. Being able to determine the electric forces inside an aggregated particle population would allow the dipole moment of each particle to be measured. A detailed and thorough analysis would characterize and identify particle polarization mechanisms.

The velocity of each particle was determined using the first five images in a recorded sequence of an aggregation when the laser was shut off. Eleven recordings were taken at varying $\mathrm{AC}$ frequencies. Figure 19 shows a set of images used for analysis, and Figure 20 shows a sample velocity map of a released aggregation spreading. 
(a)

(b)

(c)

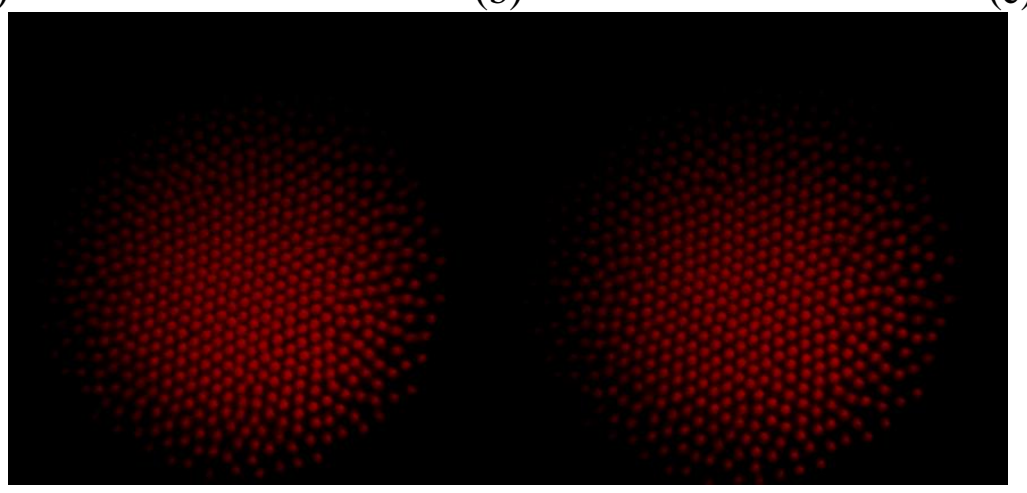

(d)

(e)

FIGURE 19 - Five images of a released aggregation at $0.033 \mathrm{~s}$ intervals. $45 \mathrm{kHz} \mathrm{AC}$, $8.811 \mathrm{~V}, 24.0 \mathrm{~mW}$ laser power.

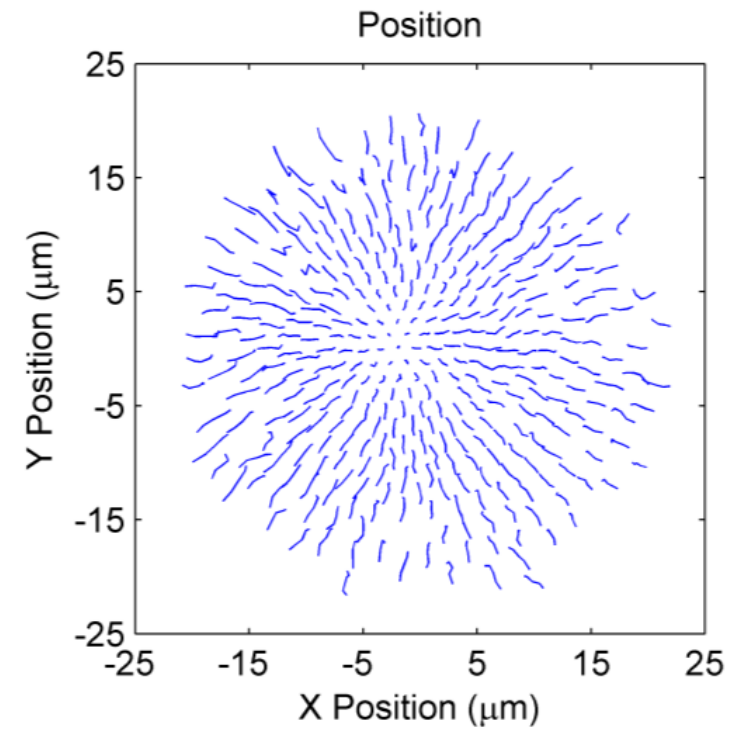

(a)

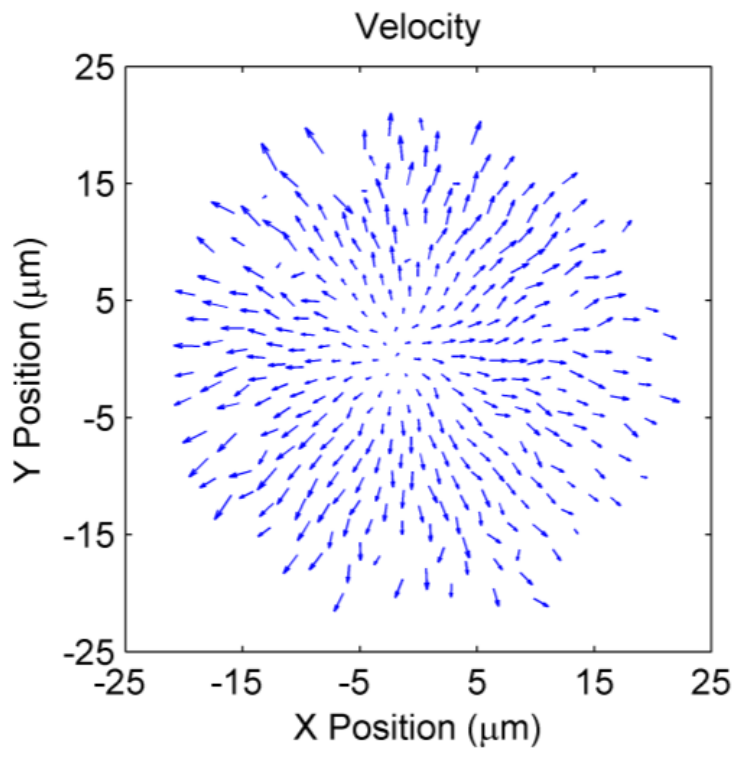

(b)

FIGURE 20 - Position and velocity of a released aggregation. $25 \mathrm{kHz}$ AC, 8.822 V, 24.0 $\mathrm{mW}$ laser power. 
Each line in Figure 20a represents the pathline of a particle. Many of the lines are not straight. As seen in Figure 20b, the velocity plot is fairly uniform, as was expected. There is more variance in particle direction for those close to the edges. The relatively low frequency used to record $(30 \mathrm{~Hz})$ was useful to average velocity to reduce random effects from ACEO and Brownian motion, but prevented capturing the acceleration of the particles. The acceleration of the particles is much faster than the frame rate used due to the very low mass of each individual particles. Figure 21 shows an acceleration plot for the same data displayed in Figure 20.

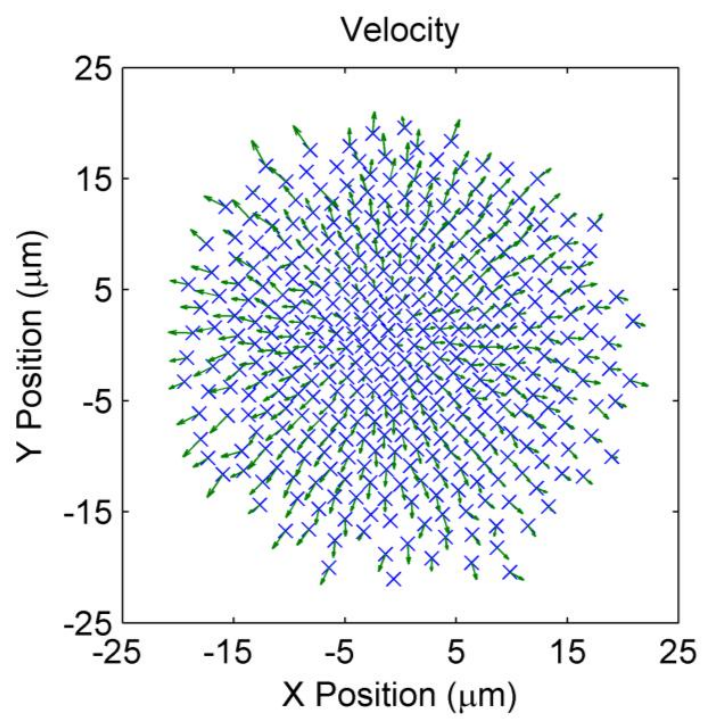

(a)

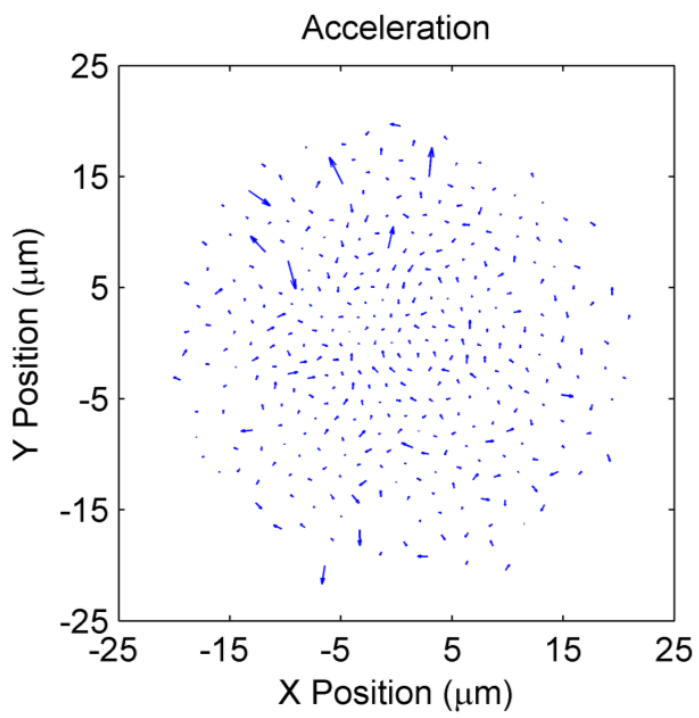

(b)

FIGURE 21 - Velocity and acceleration of a released aggregation. $25 \mathrm{kHz} \mathrm{AC}, 8.822 \mathrm{~V}$, $24.0 \mathrm{~mW}$ laser power.

As shown in Figure 21b, the acceleration of the particles appears random. The particles accelerate very quickly until the drag effect nearly-completely counteracts the electrostatic forces. This should lead to acceleration in the opposite direction as velocity; however, random acceleration due to ACEO and Brownian motion seems to be predominant. The maximum acceleration measured was on the order of $10^{-3} \mathrm{~ms}^{-2}$, demonstrating that particles are essentially at terminal velocity. 
Figure 21a shows the velocity again, this time including all of the identified data points in the third image of the five images used. This is important as it highlights the number of particles that analysis couldn't be performed on. This was due to the inability of the tracking script to follow the particles that have no velocity vectors assigned to them. For the force analysis, however, these particles were still used as they were still present in the aggregation. Figure 22 shows a plot of the speed of each particle by its radial position from the aggregation center.

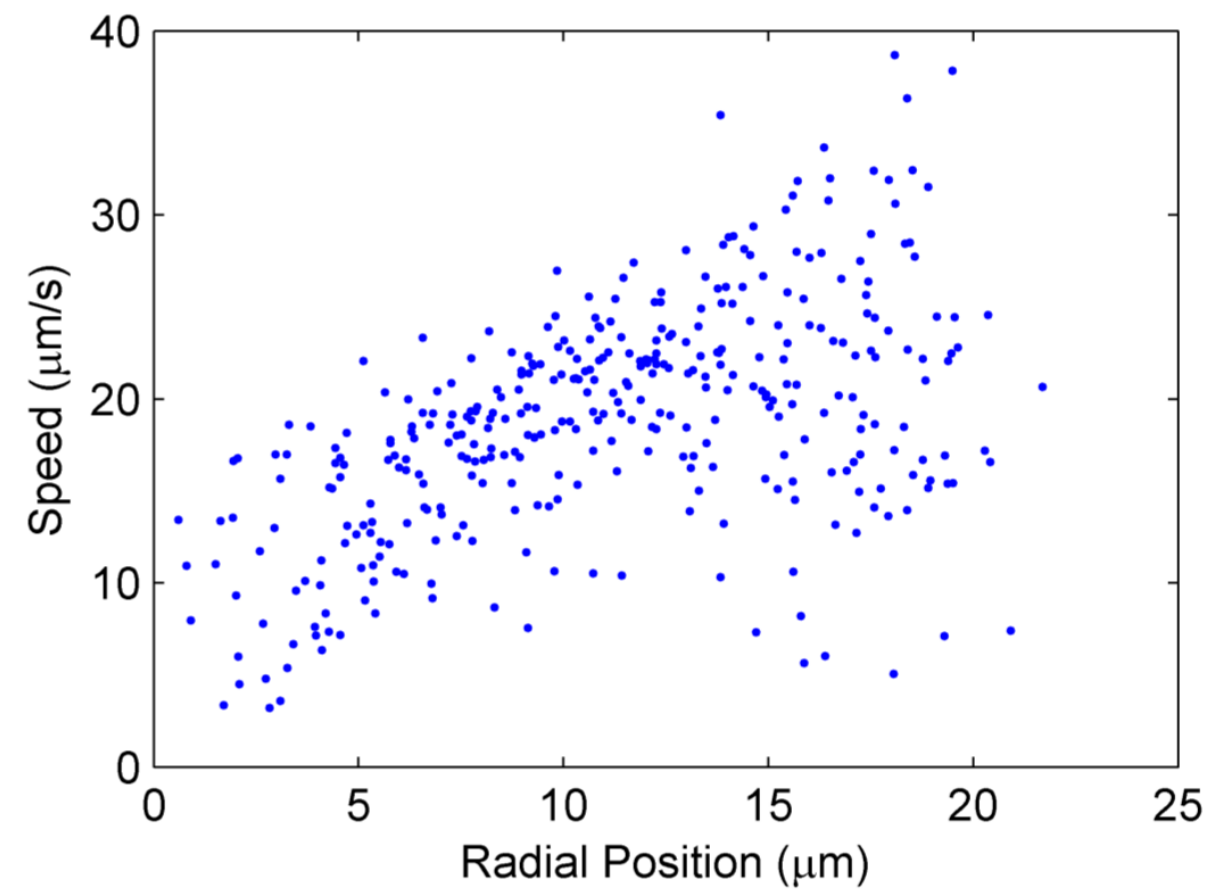

FIGURE 22 - Speed of a released aggregation by radial position. $25 \mathrm{kHz} \mathrm{AC,} 8.822 \mathrm{~V}$, $24.0 \mathrm{~mW}$ laser power.

The speed of the particles tended to increase with radial position from the aggregation center, but there was a large degree of variation. Additional data demonstrated similar trends. Aggregations have lower speed in the middle of the aggregation and higher speed at the edges where particles may freely disperse. Figure 23 
shows the average velocity by frequency, where the error bars are two standard deviations in length.

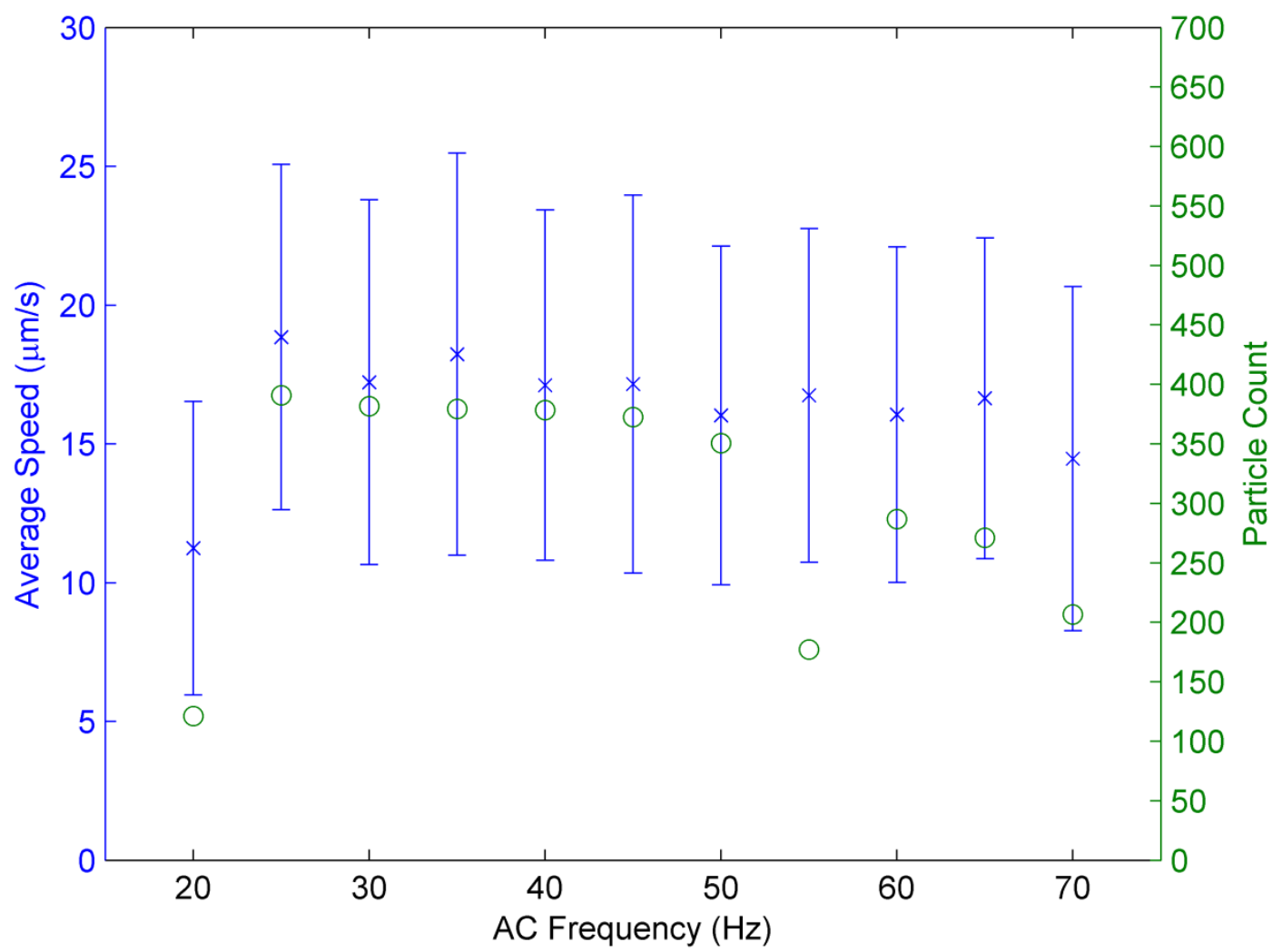

FIGURE 23 - Speed of a released aggregation by radial position. $8.758-8.822$ V, 24.0 $\mathrm{mW}$ laser power.

The particle count wasn't consistent at each data point, which most likely affected measurements. Aside from the anomalous $20 \mathrm{kHz}$ data point, the average velocity tends to decrease with increasing frequency. This is due to lower polarization at higher frequencies, as shown in Figure 11. The data isn't very consistent, making a detailed and accurate characterization of mean particle speed impractical. 


\section{Electric and Drag Force Analysis}

Force analysis on particle aggregations is of particular interest in order to develop spectroscopy, which may be achievable through observing anomalous forces in an aggregation. The force was calculated on each aggregation in the third of five frames analyzed, to correspond with the velocity calculations. A force vector map was generated for both dipole-dipole force models, shown in Figure 24.

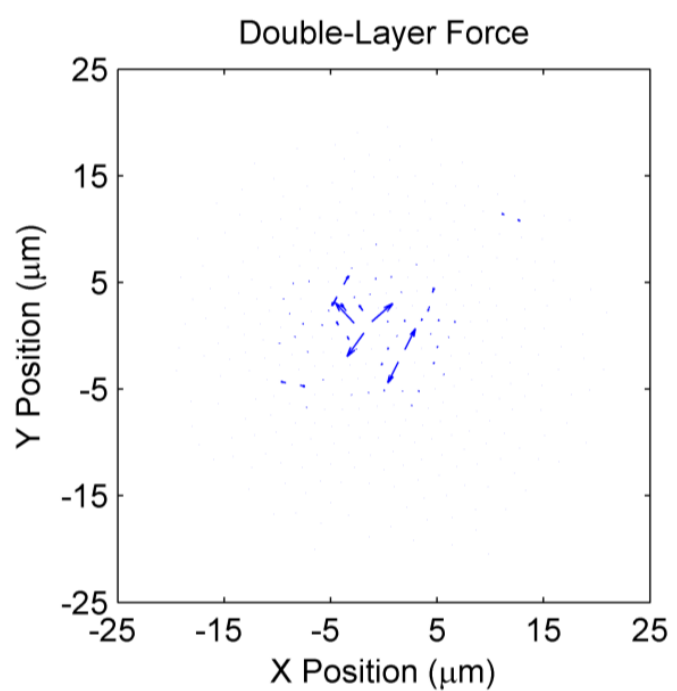

(a)

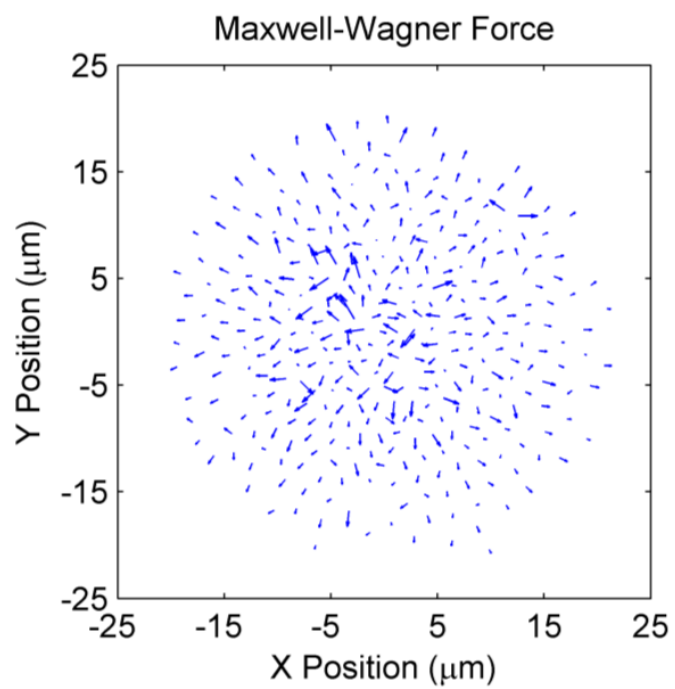

(b)

FIGURE 24 - Double-layer and Maxwell-Wagner force models. $25 \mathrm{kHz}$ AC, $8.822 \mathrm{~V}$, $24.0 \mathrm{~mW}$ laser power.

The DLVO model for the double-layer force presented an obvious problem - the variance in the magnitude of the forces from particle to particle is too high, indicating that it couldn't be responsible for the much more uniform spacing presented above. The DLVO force must act over a relatively short range, only affecting particles that are very close to each other. The model for the Maxwell-Wagner dipole-dipole repulsive force was much more evenly proportioned, but there was still a large degree of random to the force's direction. A number of methods were used to attempt to combine these forces to sum to the drag force. An attempt was made to scale and combine each individual vector 
to match the corresponding drag vector and then average the scale factors, but this yielded erroneous results. An attempt to scale each force vector by magnitude independently of its counterpart also yielded better results. Figure 25 shows the Maxwell-Wagner force scaled to match drag force, and the drag force.

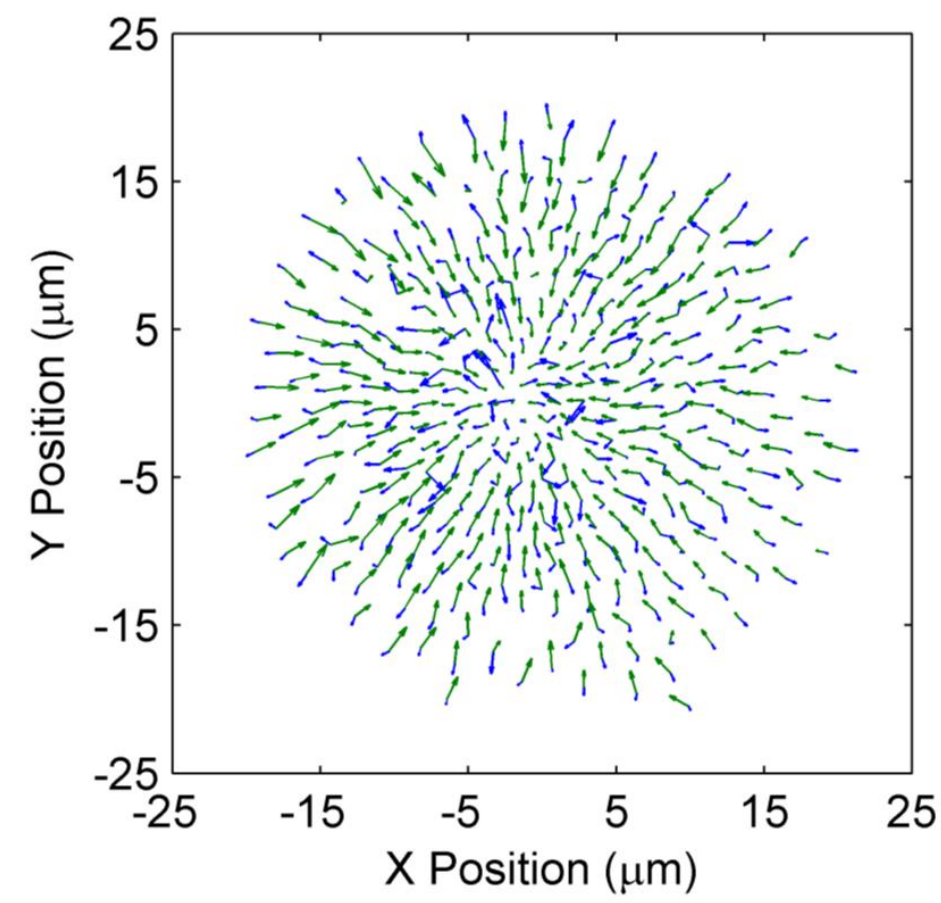

FIGURE 25 - Scaled Maxwell-Wagner forces and drag forces. $25 \mathrm{kHz}$ AC, $8.822 \mathrm{~V}$, $24.0 \mathrm{~mW}$ laser power.

If the Maxwell-Wagner repulsive forces and the drag forces matched well, they would be equal in length and opposite in direction. In some instances, this was nearly the case. In other areas (specifically near the center of the aggregation), there are relatively large forces that do not match up with the drag force. These large forces may be explained by the random motion of the particles due to ACEO and Brownian motion. These mechanisms could move particles randomly, and could have pulled the innermost particles towards each other to produce high dipole-dipole repulsive forces, while experiencing low velocity. Both the drag forces and the scaled Maxwell-Wagner 
repulsive forces indicate that the aggregation should expand under the conditions presented. While this agreement is promising, the data found indicates that both force models present a poor fit for the aggregation sampled, making force spectroscopy nonviable with current equipment. The random effects of ACEO and Brownian motion are too significant, and anomalously high or low forces could just as easily be attributed to them as opposed to a particle with an anomalously high dipole moment, rendering spectroscopy inconclusive. 


\section{CONCLUSIONS}

Of the parameters tested, the AC frequency was most effective for controlling the spacing of the aggregation. It provided the broadest range of particle-particle spacing, though the other tested parameters were still effective. Increasing the AC frequency, the laser power, and scanning speed were effective to reduce the size of the aggregation, while raising the scanning length had an adverse effect. The scanning length and speed aren't useful methods for spacing control, however, in that they are primarily useful for controlling other aspects of the aggregation's behavior. The scanning length is useful to define the geometry of the aggregation, and the scanning speed controls the behavior of the aggregation (moving dot, continuous line, or transitional state). The voltage was particularly ineffective for controlling the spacing of the aggregation. A sufficiently high voltage is required to trap the particles.

Particle spacing was also identified to be a function of the number of particles in an aggregation. Several parameters were untested in this thesis, but all remained constant

except for the particle count. A more thorough characterization would include investigating the effects of aqueous salt concentrations, salt types, particle surface chemistry, and varying aggregation size.

The particles also exhibited a minimum spacing just above what an ideal crystal would exhibit. It is hypothesized that this is due to the induced dipole in the particles, and is inevitable in REP. It is hypothesized that the Maxwell-Wagner interfacial polarization mechanism relaxed at the highest frequencies tested, but there was insufficient data to demonstrate the relaxation of a specific polarization mechanism. 
The force analysis performed was insufficient due to random acceleration of the particles. It is hypothesized that ACEO, which occurs around the base of the particles, and Brownian motion pulled the particles in random directions, which made accurate velocity and acceleration measurements difficult. Furthermore, the implementation of the electric forces presented herein proved to be insufficient for any useful analysis (such as spectroscopy), though it may be possible with different equipment. 


\section{RECOMMENDATIONS}

The effects of Brownian motion should be measured, and if random particle motion changes with voltage and frequency. The lateral motion of the particles due to ACEO should also be investigated to allow a more detailed force analysis. A higher frame rate should also allow the time scale to shrink such that change in the acceleration (or jerk) due to ACEO should be relatively small compared to the frame rate. The acceleration on the particles before and after the laser is deactivated could then be measured, and the former could be subtracted from the latter to remove the effect from consideration. However, if the acceleration of the particles due to the electric forces can be captured, it may be large enough that this is unnecessary.

A characterization of the particle count in an aggregation on the average spacing should also be conducted. Frequency characterization should also be conducted for various salts. Such characterization could also allow the measure of the salt content of an unknown medium for particles of known properties.

Spectroscopy should be possible by detecting anomalously large spacing in stagnant aggregations. This would require characterization of aggregations containing multiple particle sizes and properties. Larger particles should polarize more strongly, and should therefore force their neighboring particles further away, creating a detectible spacing that is anomalously high for the aggregation.

Frequency characterization should be repeated at higher frequencies using a lower laser power. This would determine if a polarization mechanism did actually relax at the higher frequencies tested. If one polarization mechanism can be exhibited to relax, the 
aggregation could be studied in a much simplified case as all electrostatic forces should be due to the remaining polarization mechanism.

Spectroscopy may also be possible through dynamic force analysis; where spacing may be a poor indicator, particles with differing properties may separate differently. A much more detailed force analysis and characterization would be required, however. More accurate force measurements should be acquired with a high speed camera, which may allow the acceleration of the particles to be captured. A higher frame rate of capture would also allow particle tracking to more easily follow the particles, resulting in more accurate data with less loss.

If spectroscopy is realized for dot aggregations, the next step in development should be to expand it to line aggregations in flow. It should be possible to analyze the electrical forces on particles as they flow through the vortices created in REP with bulk medium flow. This would allow rapid and inexpensive characterization of unknown colloids in a dynamic manner. 


\section{APPENDIX I}

This section contains Matlab scripts used and a block diagram of the LabVIEW VI used to control the position of the laser. The three Matlab scripts included are MasterFile.m, pcount.m, and Averager.m. The LabVIEW VI is shown in a two part image, only showing the condition for a continuously scanning line. 


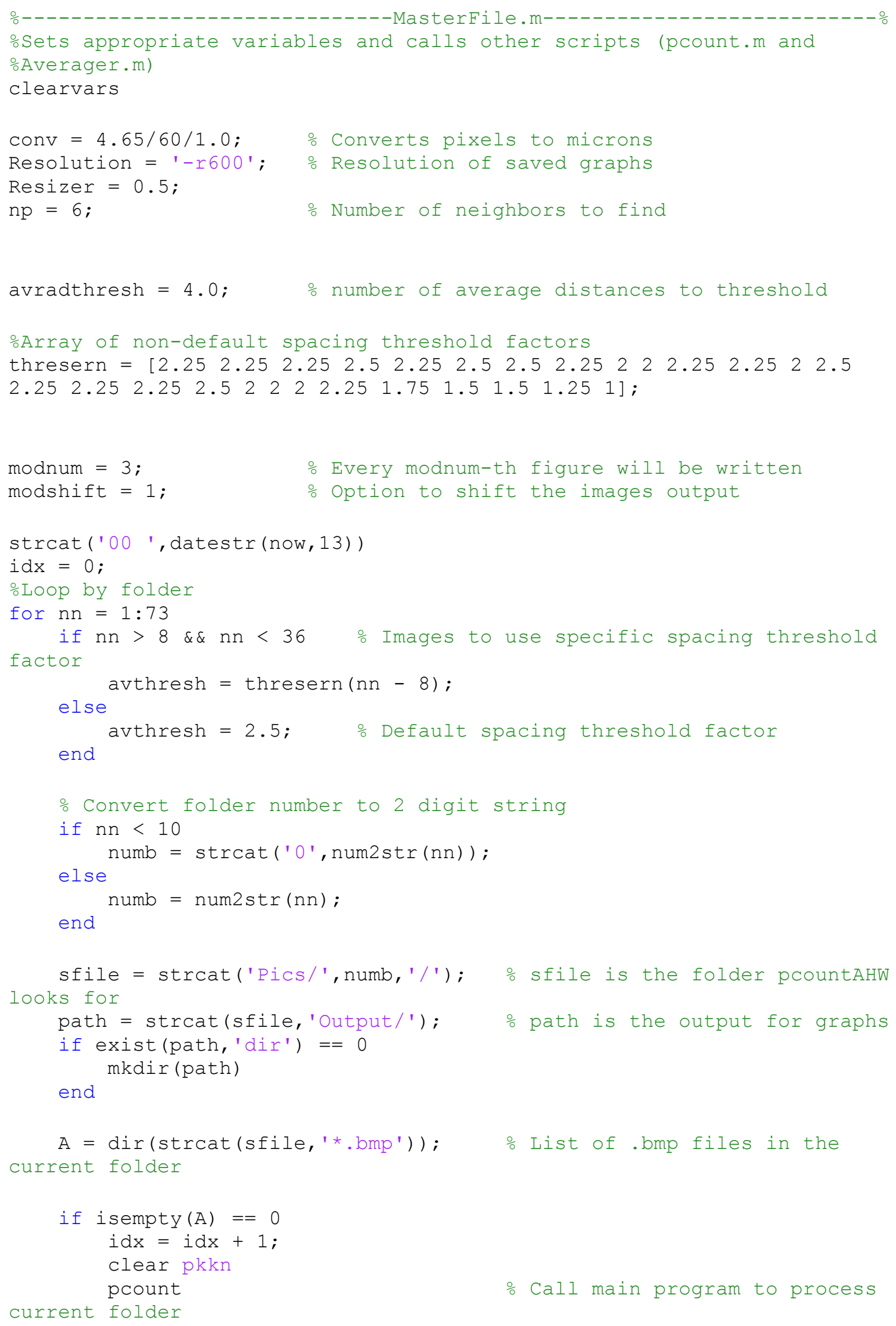




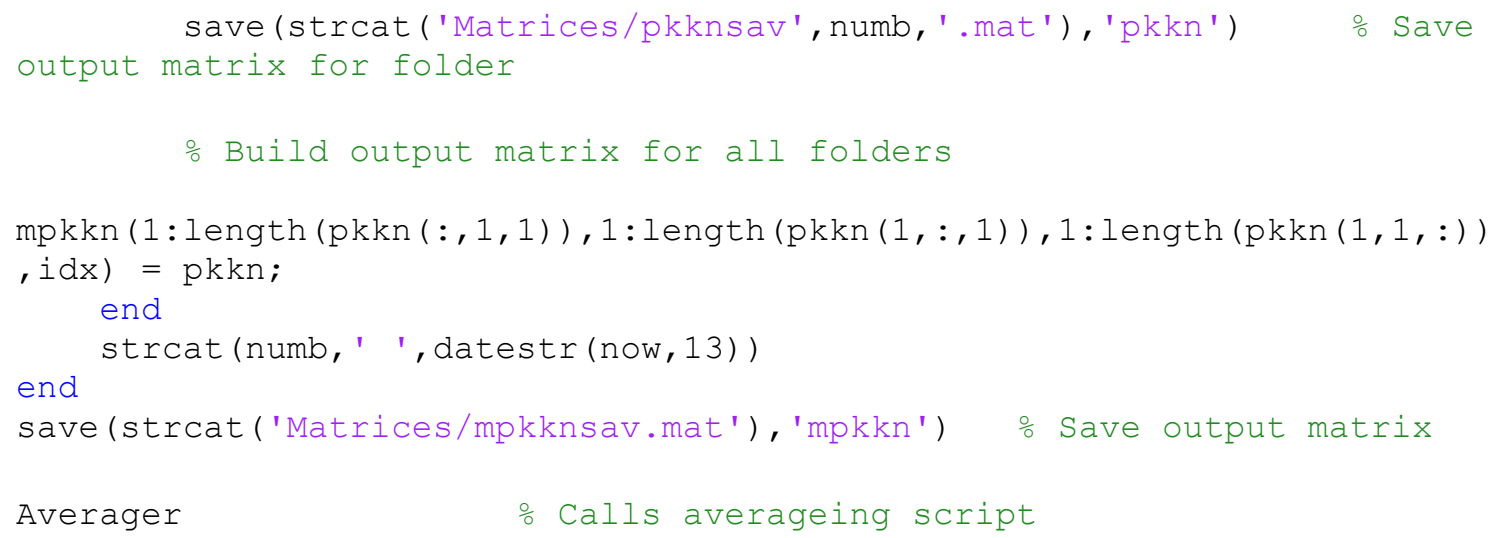




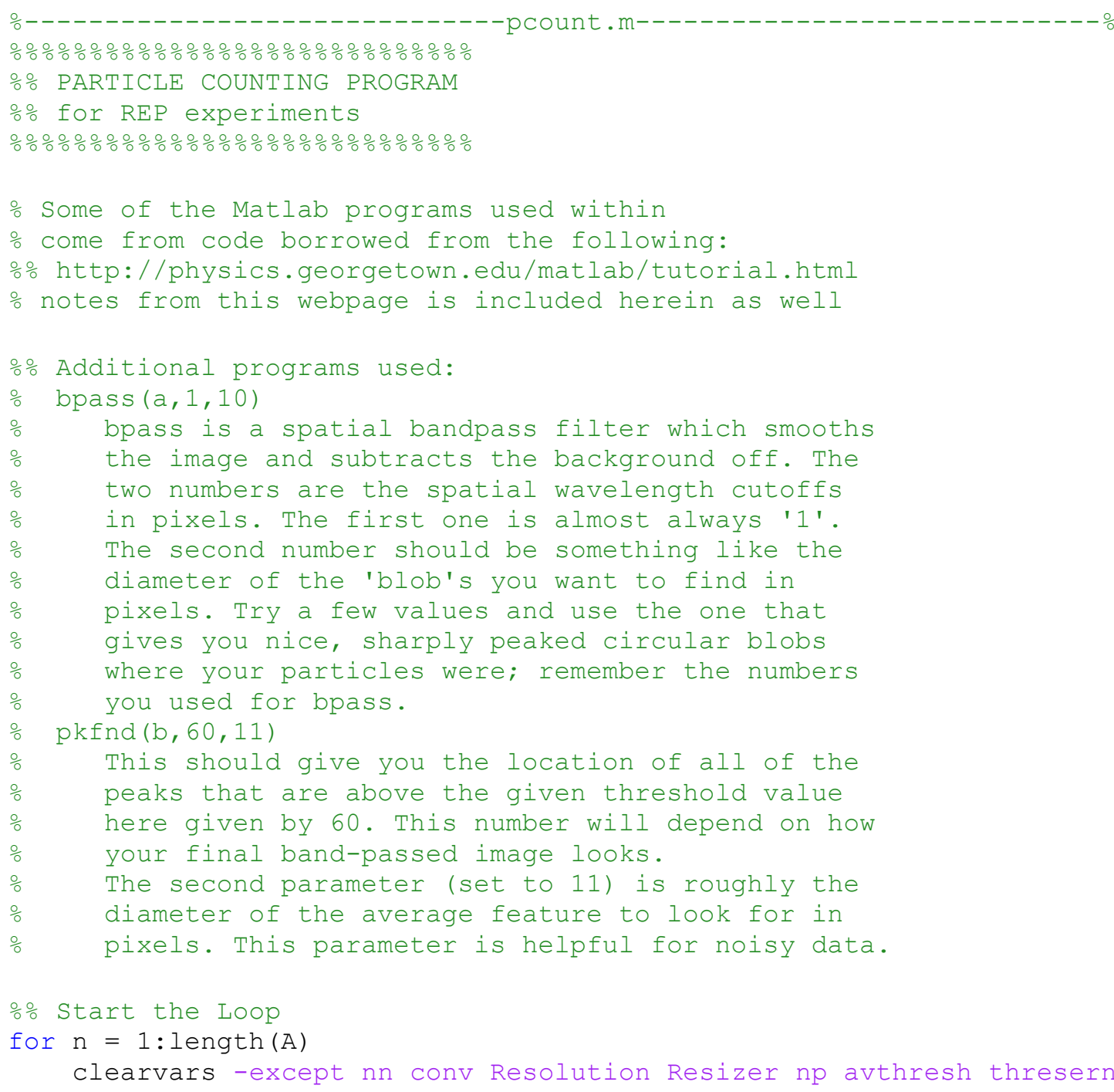
modnum modshift numb sfile path idx A mpkkn pkkn $n$ avradthresh

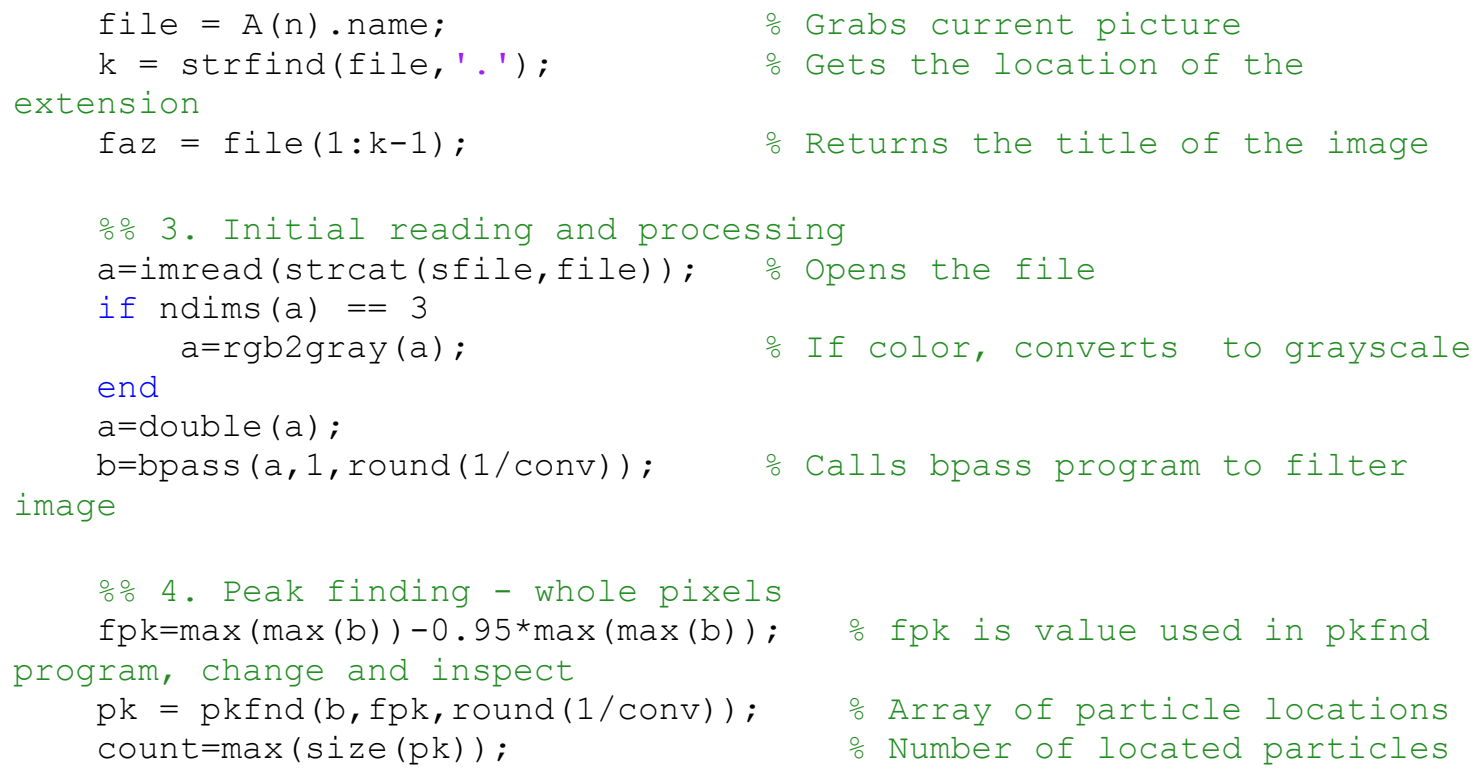




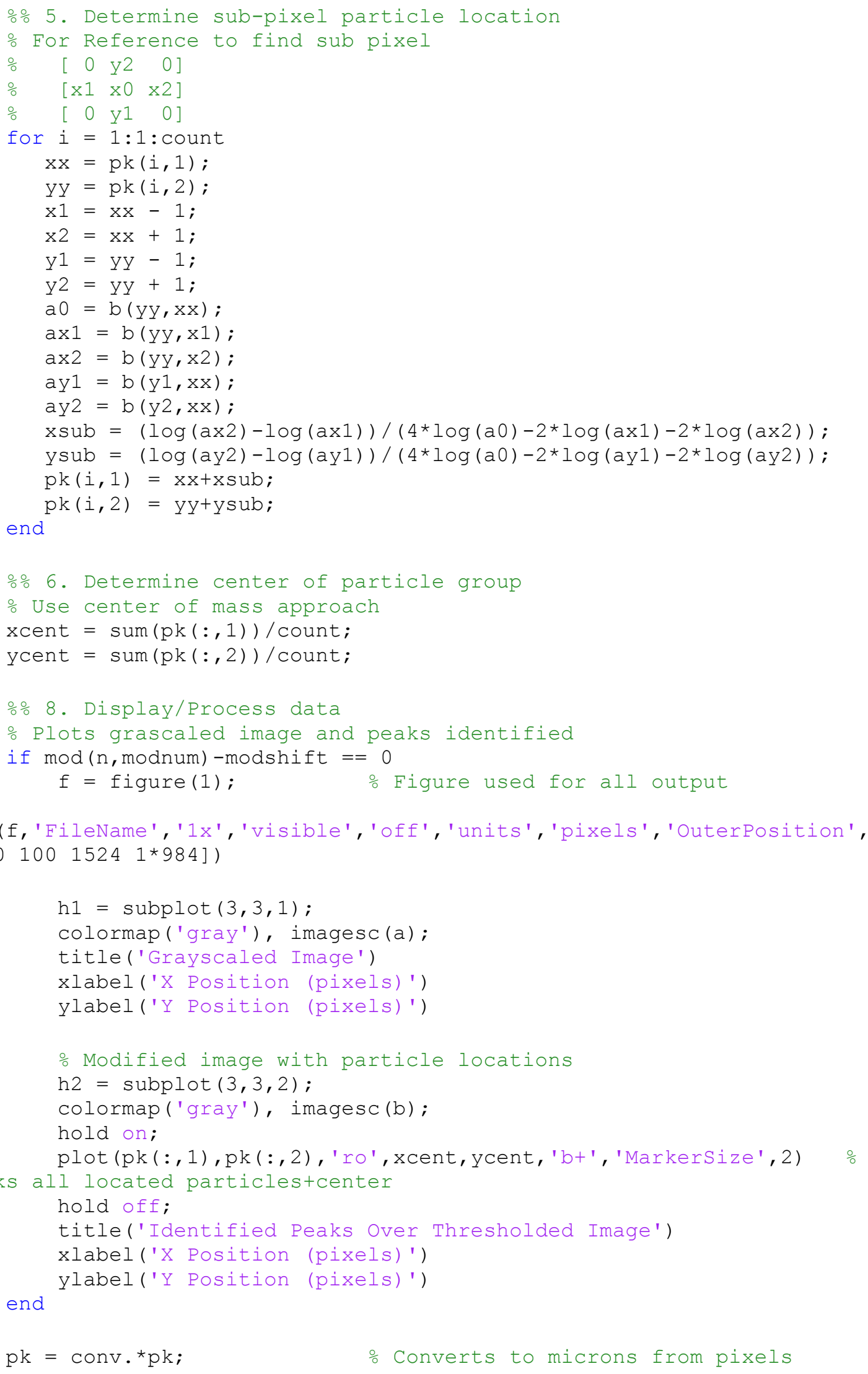




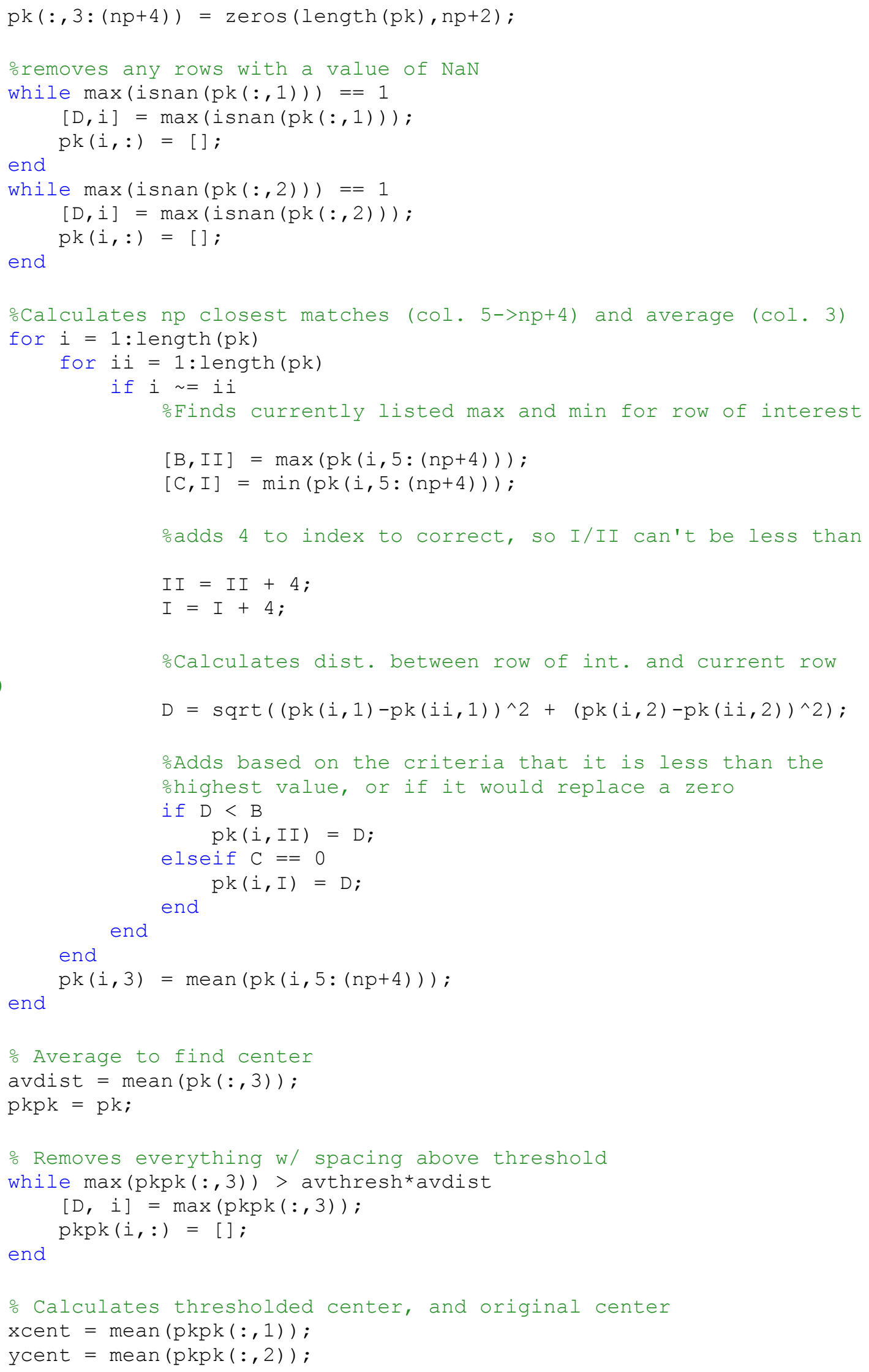




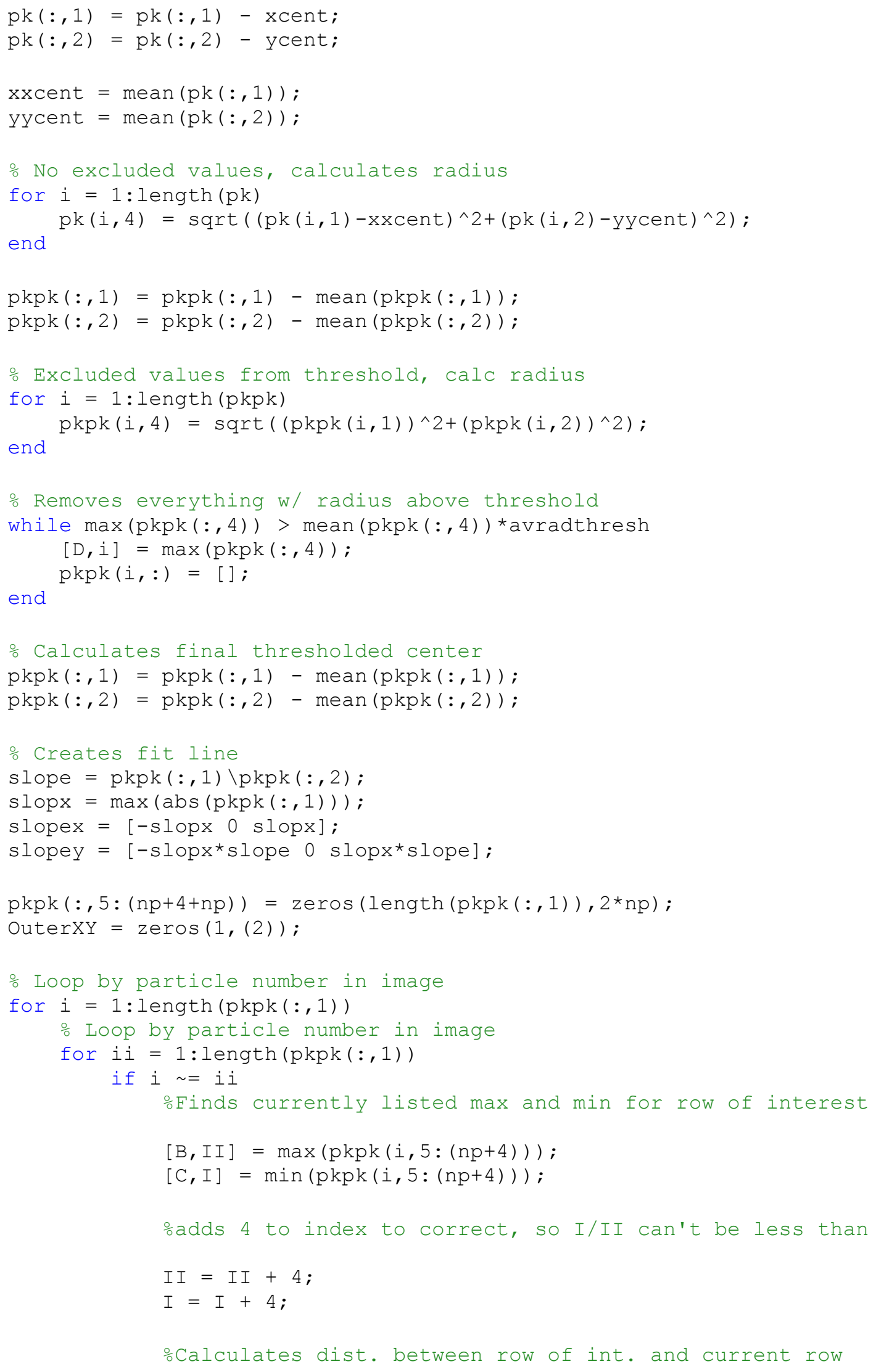


$\left.\operatorname{pkpk}(i \mathrm{i}, 2))^{\wedge} 2\right)$;

$$
D=\operatorname{sqrt}\left((\operatorname{pkpk}(i, 1)-\operatorname{pkpk}(i i, 1))^{\wedge} 2+(\operatorname{pkpk}(i, 2)-\right.
$$

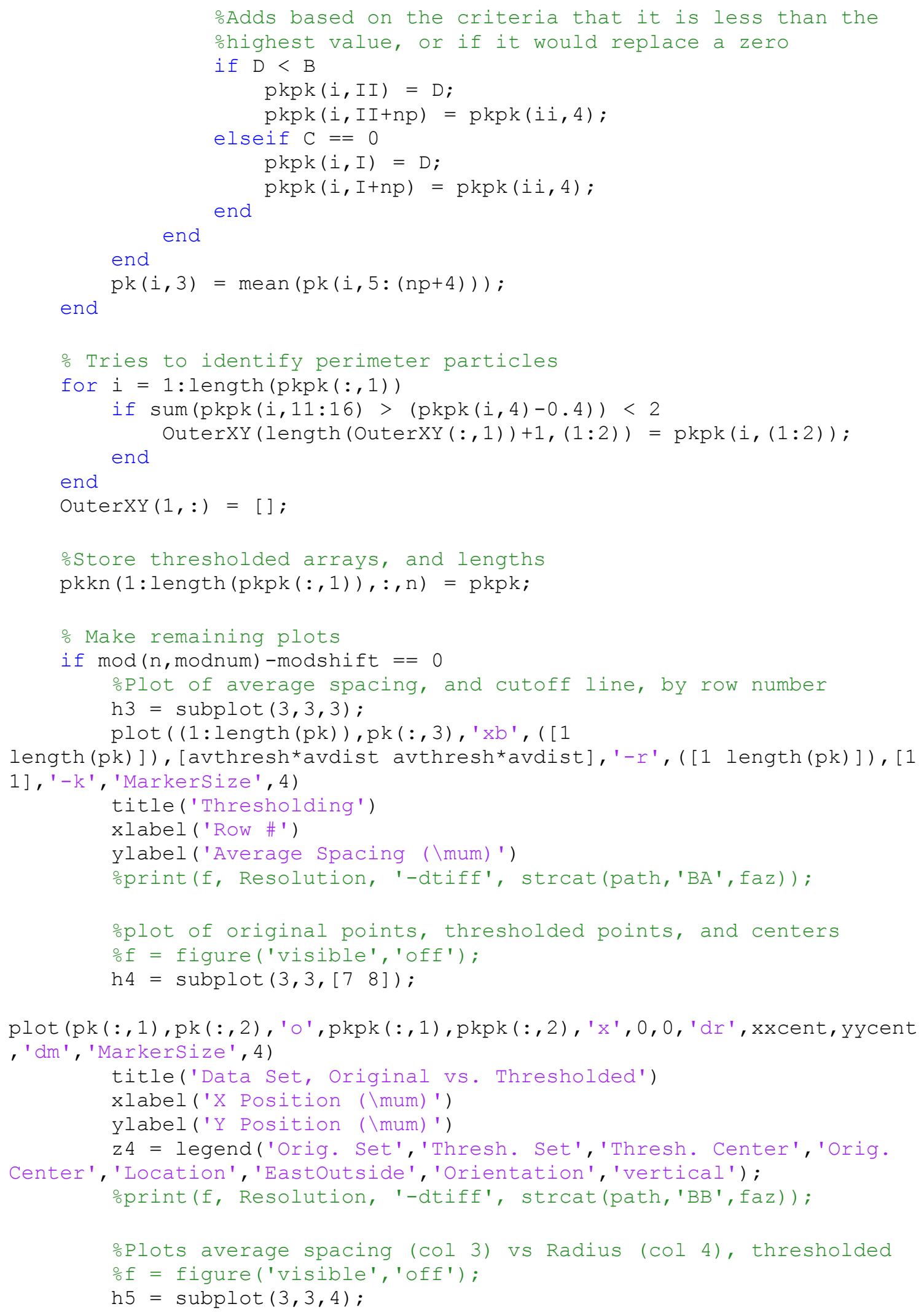

plot $\left(p k(:, 1), p k(:, 2),{ }^{\prime}{ }^{\prime}, \operatorname{pkpk}(:, 1), \operatorname{pkpk}(:, 2),{ }^{\prime} x^{\prime}, 0,0,{ }^{\prime} d r ', x x c e n t, y y c e n t\right.$ ,'dm', 'MarkerSize', 4) 


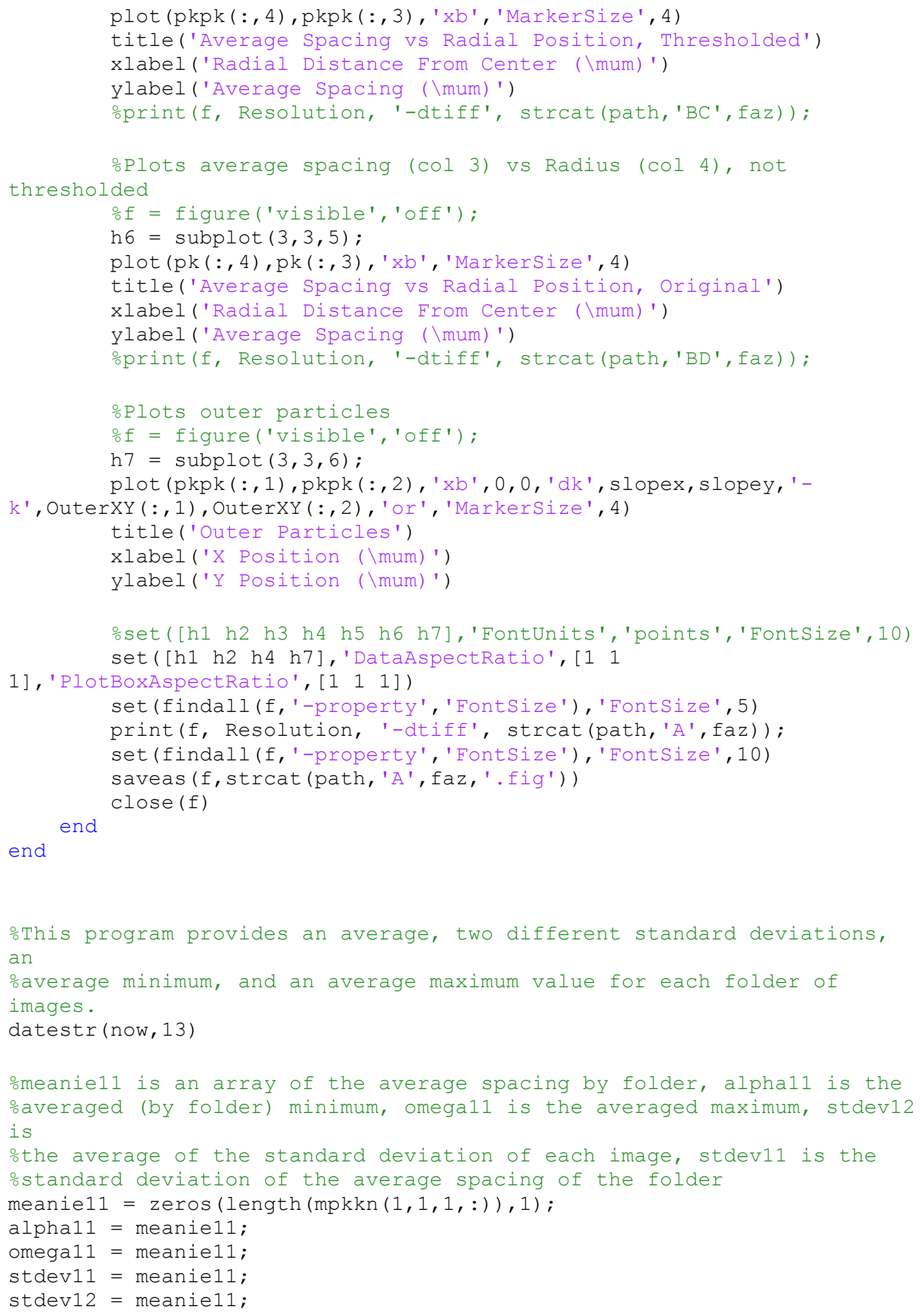




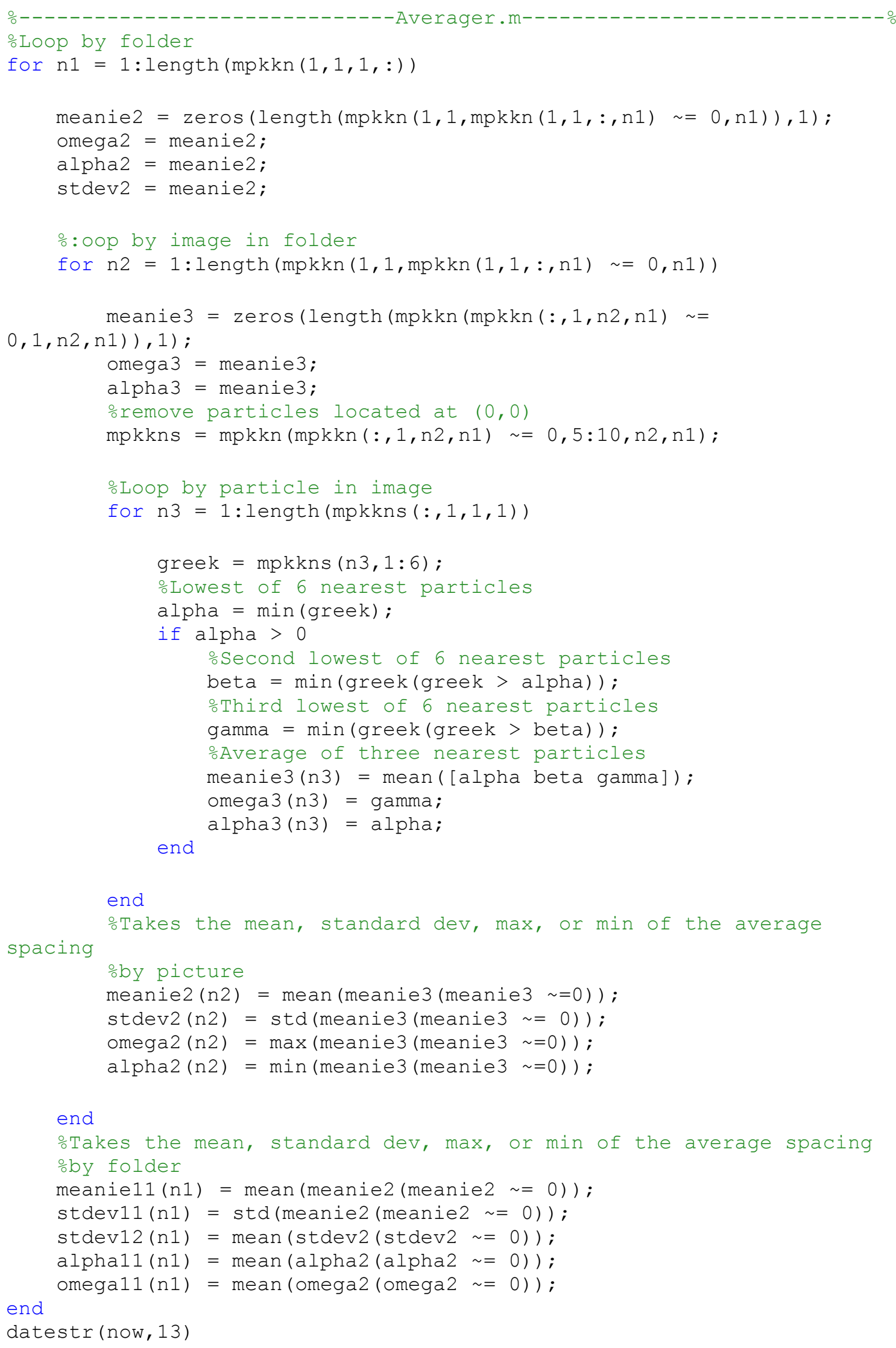




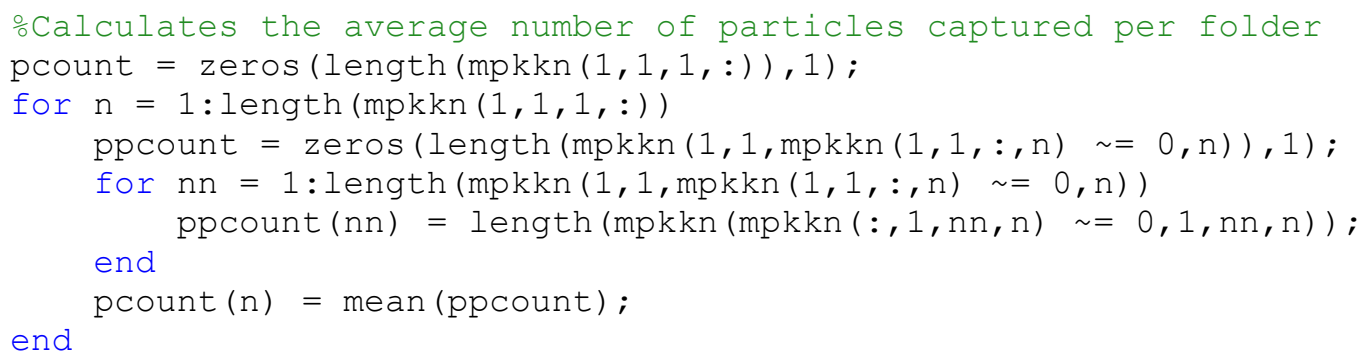




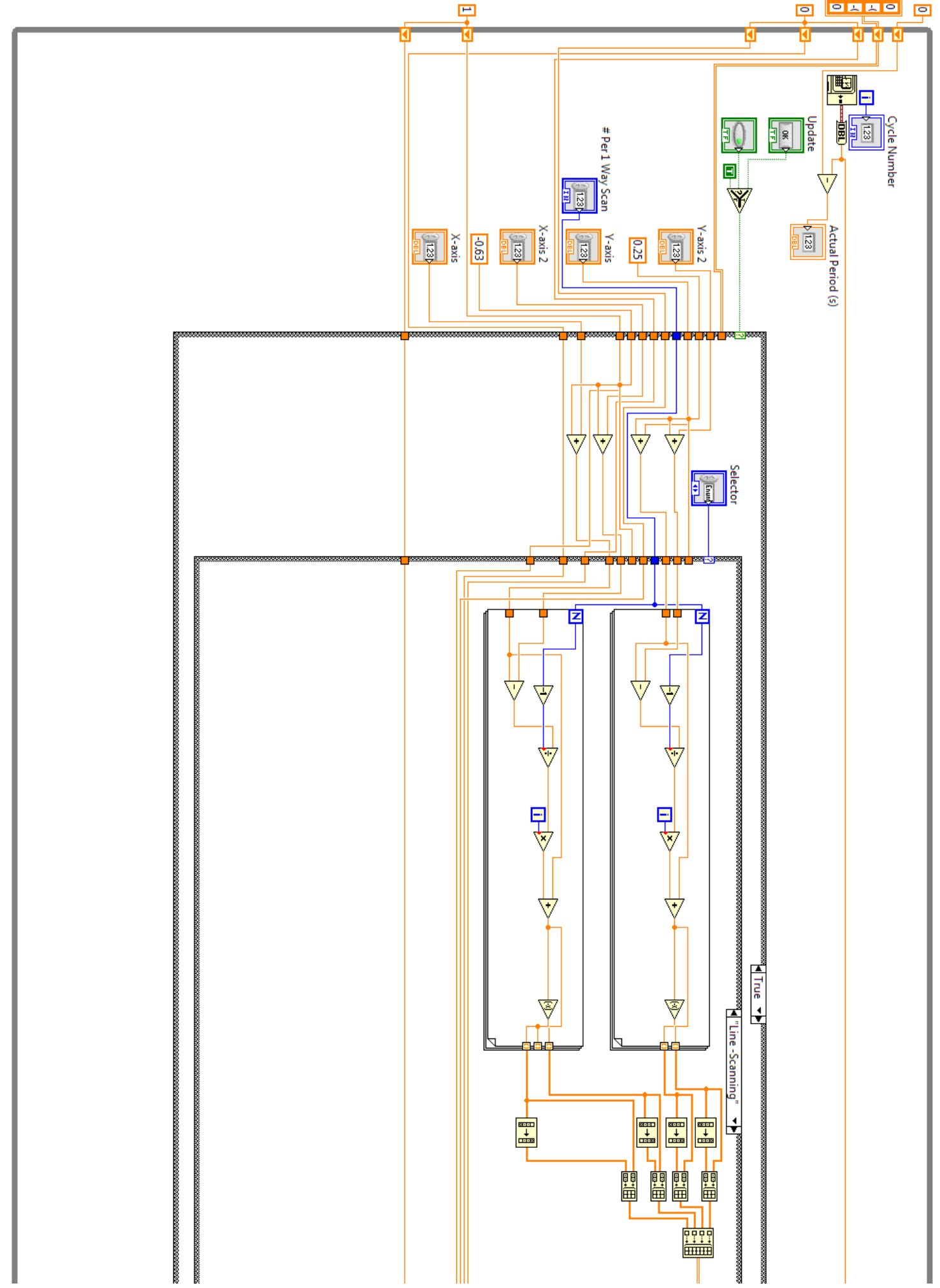




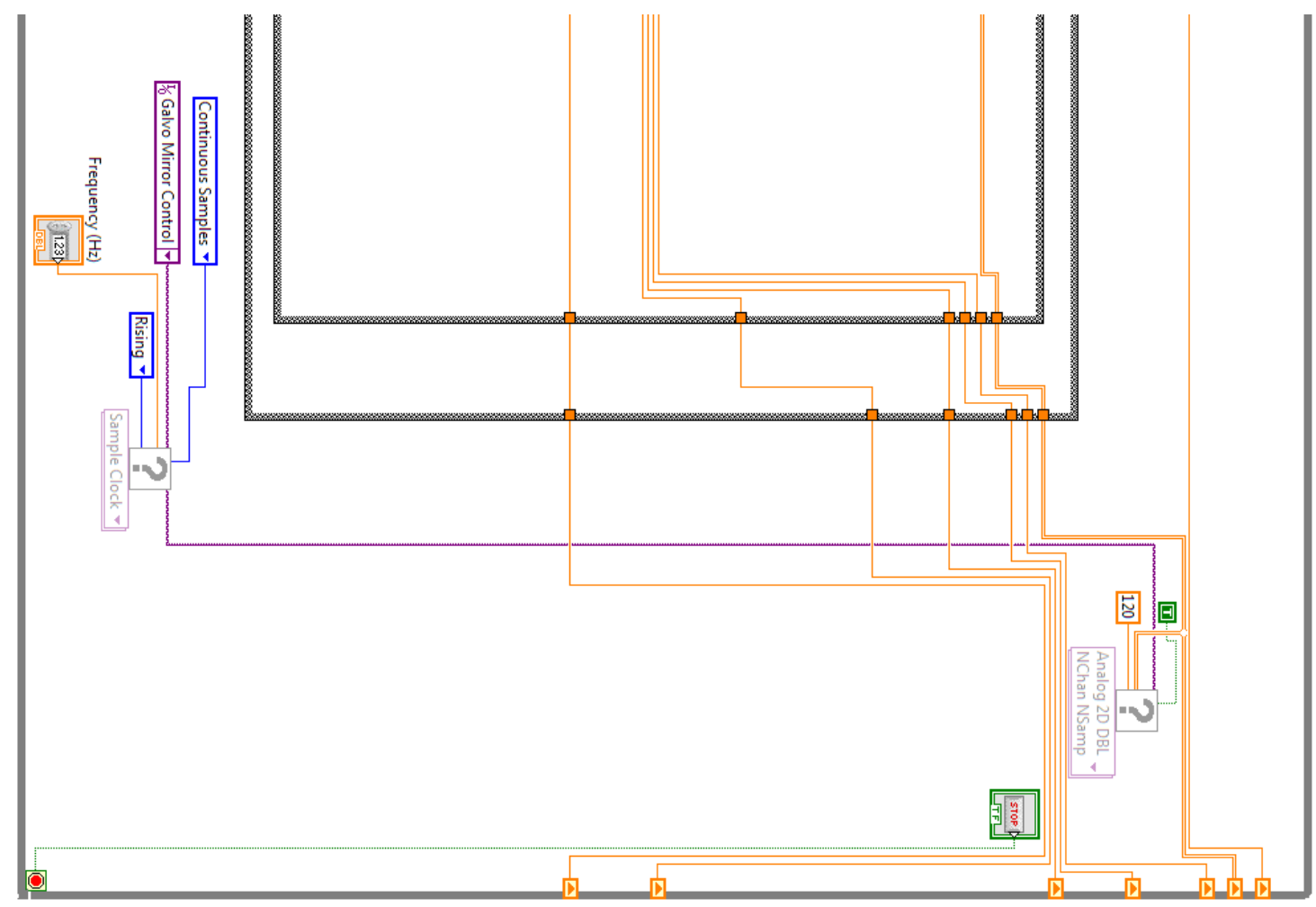




\begin{abstract}
APPENDIX II
An improved method could be implemented that would identify edge particles and interior particles, and calculate the spacing accordingly. A simple algorithm determining if a particle has any neighbors that are further from the center of the aggregation was used to little effect (see Figure A14). The algorithm used checkes to see if the particle in question is an edge particle by determining if any of the six closest neighbors have a larger radial distance to the center of the aggregation. If the particle does not have two neighbors with larger radial distances from the center, it was considered an edge particle. Adjustments were made varying the required number of particles with a greater distance, and how much greater that distance needed to be.
\end{abstract}




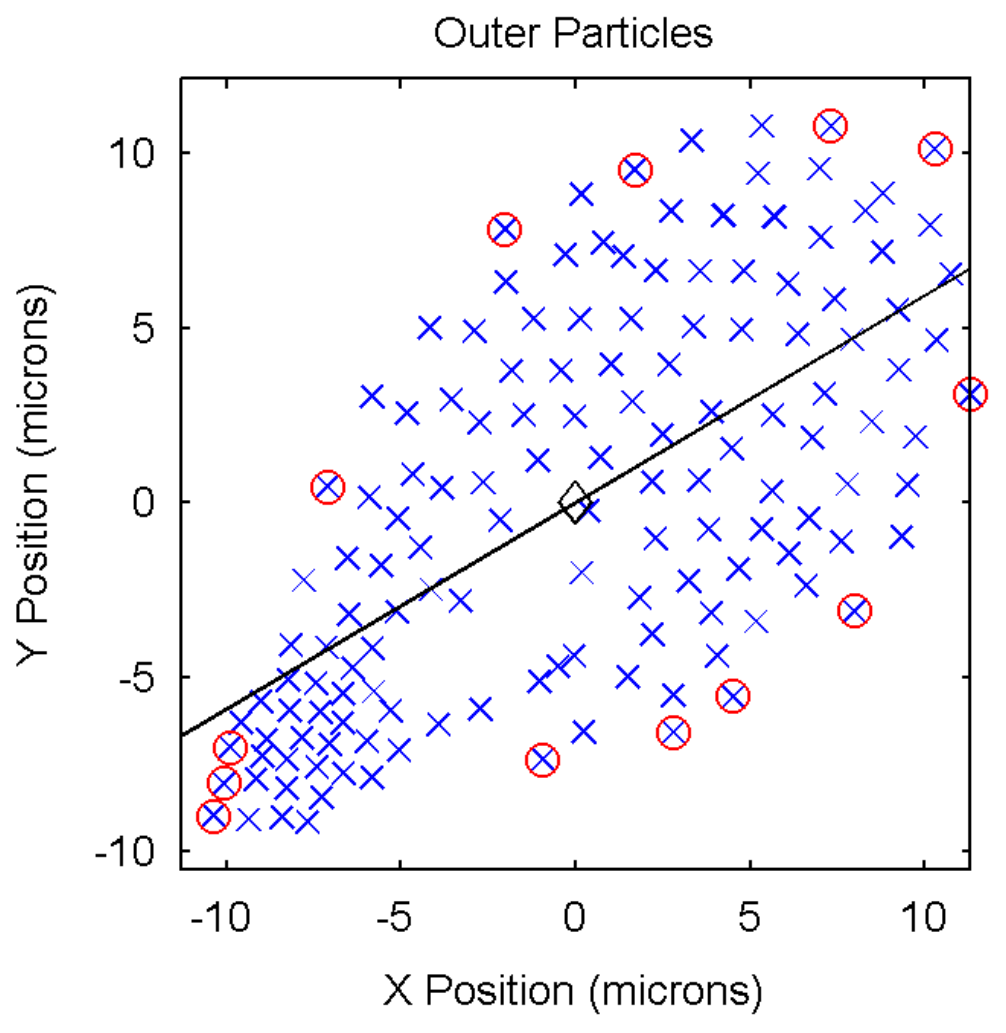

FIGURE A1 - Identified outer particles (circles) versus all particles ('x's).

As shown in Figure A14, not all edge particles were identified. Choosing to require a fixed number of particles with a larger radial distance from the center of the aggregation yielded inconsistent results, and could not be relied upon. Quality of the edge detection varied significantly; sometimes interior points were selected, sometimes most of the exterior points were selected. Never were all of the exterior points selected. Edge particles could be determined for a small number of images manually; for the number of images processed for this thesis, this was not feasible. Improved results could be obtained by developing a suitable algorithm to determine outer particles in order to calculate their spacing separately from interior particles. More results are shown in Appendix III. 


\begin{abstract}
APPENDIX III
This section presents additional results not shown in the main text. The first section consists of tables of all data used in the thesis. The first figure contains spacing by radius for dot-shaped aggregations, listing property values by experiment number. The remaining figures show selected experimental data analysis from the data collected.
\end{abstract}


TABLE AI

USED DATA COLLECTED ON 6/15/12, 1-52

\begin{tabular}{|c|c|c|c|c|c|c|c|c|}
\hline$\#$ & $\begin{array}{c}\text { Voltage } \\
\text { (V) }\end{array}$ & $\begin{array}{c}\text { AC } \\
\text { Freq. } \\
\text { (kHz) }\end{array}$ & $\begin{array}{c}\text { Laser } \\
\text { Current } \\
(\mathbf{m A})\end{array}$ & $\begin{array}{l}\text { Laser } \\
\text { Power } \\
(\mathbf{m W})\end{array}$ & $\begin{array}{l}\text { Light } \\
\text { Filter }\end{array}$ & $\begin{array}{c}\text { Length } \\
(\mu \mathrm{m})\end{array}$ & $\begin{array}{c}\text { Scan } \\
\text { Freq. } \\
(\mathbf{H z})\end{array}$ & Type \\
\hline 1 & 6.533 & 30 & 94.1 & 11.8468 & 0.35 & 0 & 0 & N/A. \\
\hline 2 & 6.533 & 30 & 94.1 & 11.8468 & 0.35 & 11.8576 & 16 & Cont. \\
\hline 3 & 6.533 & 30 & 94.1 & 11.8468 & 0.35 & 23.7152 & 16 & Cont. \\
\hline 4 & 6.533 & 30 & 94.1 & 11.8468 & 0.35 & 35.5728 & 16 & Cont. \\
\hline 5 & 6.533 & 30 & 94.1 & 11.8468 & 0.35 & 47.4304 & 16 & Cont. \\
\hline 6 & 6.533 & 30 & 94.1 & 11.8468 & 0.35 & 59.288 & 16 & Cont. \\
\hline 7 & 6.533 & 30 & 94.1 & 11.8468 & 0.35 & 71.1456 & 16 & Cont. \\
\hline 8 & 6.533 & 30 & 94.1 & 11.8468 & 0.35 & 83.0032 & 16 & Cont. \\
\hline 9 & 6.555 & 20 & 94.1 & 11.8468 & 0.35 & 59.288 & 16 & Cont. \\
\hline 10 & 6.551 & 22 & 94.1 & 11.8468 & 0.35 & 59.288 & 16 & Cont. \\
\hline 11 & 6.547 & 24 & 94.1 & 11.8468 & 0.35 & 59.288 & 16 & Cont. \\
\hline 12 & 6.547 & 26 & 94.1 & 11.8468 & 0.35 & 59.288 & 16 & Cont. \\
\hline 13 & 6.544 & 28 & 94.1 & 11.8468 & 0.35 & 59.288 & 16 & Cont. \\
\hline 14 & 6.54 & 30 & 94.1 & 11.8468 & 0.35 & 59.288 & 16 & Cont. \\
\hline 15 & 6.538 & 32 & 94.1 & 11.8468 & 0.35 & 59.288 & 16 & Cont. \\
\hline 16 & 6.539 & 34 & 94.1 & 11.8468 & 0.35 & 59.288 & 16 & Cont. \\
\hline 17 & 6.537 & 36 & 94.1 & 11.8468 & 0.35 & 59.288 & 16 & Cont. \\
\hline 18 & 6.54 & 38 & 94.1 & 11.8468 & 0.35 & 59.288 & 16 & Cont. \\
\hline 19 & 6.539 & 40 & 94.1 & 11.8468 & 0.35 & 59.288 & 16 & Cont. \\
\hline 20 & 6.537 & 42 & 94.1 & 11.8468 & 0.35 & 59.288 & 16 & Cont. \\
\hline 21 & 6.537 & 44 & 94.1 & 11.8468 & 0.35 & 59.288 & 16 & Cont. \\
\hline 22 & 6.536 & 46 & 94.1 & 11.8468 & 0.35 & 59.288 & 16 & Cont. \\
\hline 23 & 6.535 & 48 & 94.1 & 11.8468 & 0.35 & 59.288 & 16 & Cont. \\
\hline 24 & 6.538 & 50 & 94.1 & 11.8468 & 0.35 & 59.288 & 16 & Cont. \\
\hline 25 & 6.544 & 52 & 94.1 & 11.8468 & 0.35 & 59.288 & 16 & Cont. \\
\hline 26 & 6.548 & 54 & 94.1 & 11.8468 & 0.35 & 59.288 & 16 & Cont. \\
\hline 27 & 6.551 & 56 & 94.1 & 11.8468 & 0.35 & 59.288 & 16 & Cont. \\
\hline 28 & 6.552 & 58 & 94.1 & 11.8468 & 0.35 & 59.288 & 16 & Cont. \\
\hline 29 & 6.552 & 60 & 94.1 & 11.8468 & 0.35 & 59.288 & 16 & Cont. \\
\hline 30 & 6.555 & 62 & 94.1 & 11.8468 & 0.35 & 59.288 & 16 & Cont. \\
\hline 31 & 6.555 & 64 & 94.1 & 11.8468 & 0.35 & 59.288 & 16 & Cont. \\
\hline 32 & 6.557 & 66 & 94.1 & 11.8468 & 0.35 & 59.288 & 16 & Cont. \\
\hline 33 & 6.563 & 68 & 94.1 & 11.8468 & 0.35 & 59.288 & 16 & Cont. \\
\hline 34 & 6.562 & 70 & 94.1 & 11.8468 & 0.35 & 59.288 & 16 & Cont. \\
\hline 35 & 6.563 & 72 & 94.1 & 11.8468 & 0.35 & 59.288 & 16 & Cont. \\
\hline 36 & 6.573 & 30 & 94.1 & 11.8468 & 0.35 & 59.288 & 16 & Cont. \\
\hline 37 & 6.566 & 30 & 96.8 & 12.3949 & 0.35 & 59.288 & 16 & Cont. \\
\hline 38 & 6.561 & 30 & 99.2 & 12.8821 & 0.35 & 59.288 & 16 & Cont. \\
\hline 39 & 6.559 & 30 & 101.4 & 13.3287 & 0.35 & 59.288 & 16 & Cont. \\
\hline 40 & 6.558 & 30 & 102.9 & 13.6332 & 0.35 & 59.288 & 16 & Cont. \\
\hline 41 & 6.556 & 30 & 104 & 13.8565 & 0.35 & 59.288 & 16 & Cont. \\
\hline 42 & 6.554 & 30 & 105.3 & 14.1204 & 0.35 & 59.288 & 16 & Cont. \\
\hline 43 & 6.553 & 30 & 107 & 14.4655 & 0.35 & 59.288 & 16 & Cont. \\
\hline 44 & 6.553 & 30 & 109 & 14.8715 & 0.35 & 59.288 & 16 & Cont. \\
\hline 45 & 6.551 & 30 & 110.6 & 15.1963 & 0.35 & 59.288 & 16 & Cont. \\
\hline 46 & 6.55 & 30 & 112.5 & 15.582 & 0.35 & 59.288 & 16 & Cont. \\
\hline 47 & 6.551 & 30 & 115.7 & 16.2316 & 0.35 & 59.288 & 16 & Cont. \\
\hline 48 & 6.551 & 30 & 118.2 & 16.7391 & 0.35 & 59.288 & 16 & Cont. \\
\hline 49 & 6.551 & 30 & 121 & 17.3075 & 0.35 & 59.288 & 16 & Cont. \\
\hline 50 & 6.55 & 30 & 124.1 & 17.9368 & 0.35 & 59.288 & 16 & Cont. \\
\hline 51 & 6.551 & 30 & 124.1 & 17.9368 & 0.35 & 59.288 & 16 & Cont. \\
\hline 52 & 6.551 & 30 & 128.1 & 18.7488 & 0.35 & 59.288 & 16 & Cont. \\
\hline
\end{tabular}


TABLE AII

USED DATA COLLECTED ON 6/13/12, 53-64

\begin{tabular}{ccccccccc}
\hline$\#$ & $\begin{array}{c}\text { Voltage } \\
(\mathbf{V})\end{array}$ & $\begin{array}{c}\text { AC } \\
\text { Freq. } \\
\mathbf{( k H z})\end{array}$ & $\begin{array}{c}\text { Laser } \\
\text { Current } \\
(\mathbf{m A})\end{array}$ & $\begin{array}{c}\text { Laser } \\
\text { Power } \\
(\mathbf{m W})\end{array}$ & $\begin{array}{c}\text { Light } \\
\text { Filter }\end{array}$ & $\begin{array}{c}\text { Length } \\
(\boldsymbol{\mu m})\end{array}$ & $\begin{array}{c}\text { Scan } \\
\text { Freq. } \\
(\mathbf{H z})\end{array}$ & Type \\
\hline $\mathbf{5 3}$ & 6.3577 & 30 & 77.7 & 24.336 & N/A & 53.3592 & 0.375 & Cont. \\
$\mathbf{5 4}$ & 6.3577 & 30 & 77.7 & 24.336 & N/A & 53.3592 & 0.5 & Cont. \\
$\mathbf{5 5}$ & 6.354 & 30 & 77.7 & 24.336 & N/A & 53.3592 & 0.75 & Cont. \\
$\mathbf{5 6}$ & 6.352 & 30 & 77.7 & 24.336 & N/A & 53.3592 & 1 & Cont. \\
$\mathbf{5 7}$ & 6.351 & 30 & 77.7 & 24.336 & N/A & 53.3592 & 1.25 & Cont. \\
$\mathbf{5 8}$ & 6.35 & 30 & 77.7 & 24.336 & N/A & 53.3592 & 1.5 & Cont. \\
$\mathbf{5 9}$ & 6.348 & 30 & 77.7 & 24.336 & N/A & 53.3592 & 2 & Cont. \\
$\mathbf{6 0}$ & 6.3467 & 30 & 77.7 & 24.336 & N/A & 53.3592 & 3 & Cont. \\
$\mathbf{6 1}$ & 6.345 & 30 & 77.7 & 24.336 & N/A & 53.3592 & 4 & Cont. \\
$\mathbf{6 2}$ & 6.343 & 30 & 77.7 & 24.336 & N/A & 53.3592 & 6 & Cont. \\
$\mathbf{6 3}$ & 6.342 & 30 & 77.7 & 24.336 & N/A & 53.3592 & 8 & Cont. \\
$\mathbf{6 4}$ & 6.341 & 30 & 77.7 & 24.336 & N/A & 53.3592 & 16 & Cont. \\
\hline
\end{tabular}

TABLE AIII

USED DATA COLLECTED ON 6/04/12, 65-73

\begin{tabular}{ccccccccc}
\hline$\#$ & $\begin{array}{c}\text { Voltage } \\
(\mathbf{V})\end{array}$ & $\begin{array}{c}\text { AC } \\
\text { Freq. } \\
(\mathbf{k H z})\end{array}$ & $\begin{array}{c}\text { Laser } \\
\text { Current } \\
(\mathbf{m A})\end{array}$ & $\begin{array}{c}\text { Laser } \\
\text { Power } \\
(\mathbf{m W})\end{array}$ & $\begin{array}{c}\text { Light } \\
\text { Filter }\end{array}$ & $\begin{array}{c}\text { Length } \\
(\boldsymbol{\mu m})\end{array}$ & $\begin{array}{c}\text { Scan } \\
\text { Freq. } \\
(\mathbf{H z})\end{array}$ & Type \\
\hline $\mathbf{6 5}$ & 6.4624 & 60 & 81.6 & 26.598 & N/A & 77.0744 & 30 & Type. \\
\hline $\mathbf{6 6}$ & 5.8249 & 60 & 81.6 & 26.598 & N/A & 77.0744 & 30 & Type. \\
$\mathbf{6 7}$ & 5.1806 & 60 & 81.6 & 26.598 & N/A & 77.0744 & 30 & Type. \\
$\mathbf{6 8}$ & 4.5352 & 60 & 81.6 & 26.598 & N/A & 77.0744 & 30 & Type. \\
$\mathbf{6 9}$ & 3.8887 & 60 & 81.6 & 26.598 & N/A & 77.0744 & 30 & Type. \\
\hline $\mathbf{7 0}$ & 6.1438 & 60 & 81.6 & 26.598 & N/A & 77.0744 & 30 & Type. \\
$\mathbf{7 1}$ & 5.4996 & 60 & 81.6 & 26.598 & N/A & 77.0744 & 30 & Type. \\
$\mathbf{7 2}$ & 4.8551 & 60 & 81.6 & 26.598 & N/A & 77.0744 & 30 & Type. \\
$\mathbf{7 3}$ & 4.2103 & 60 & 81.6 & 26.598 & N/A & 77.0744 & 30 & Type. \\
\hline
\end{tabular}


TABLE AIV

USED DATA COLLECTED ON 5/30/12, 74-84

\begin{tabular}{ccccccccc}
\hline$\#$ & $\begin{array}{c}\text { Voltage } \\
(\mathbf{V})\end{array}$ & $\begin{array}{c}\text { AC } \\
\text { Freq. } \\
(\mathbf{k H z})\end{array}$ & $\begin{array}{c}\text { Laser } \\
\text { Current } \\
(\mathbf{m A})\end{array}$ & $\begin{array}{c}\text { Laser } \\
\text { Power } \\
(\mathbf{m W})\end{array}$ & $\begin{array}{c}\text { Light } \\
\text { Filter }\end{array}$ & $\begin{array}{c}\text { Length } \\
(\boldsymbol{\mu m})\end{array}$ & $\begin{array}{c}\text { Scan } \\
\text { Freq. } \\
(\mathbf{H z})\end{array}$ & Type \\
\hline $\mathbf{7 4}$ & 8.7956 & 50 & 77.1 & 23.988 & N/A & 0 & 0 & N/A \\
$\mathbf{7 5}$ & 8.811 & 45 & 77.1 & 23.988 & N/A & 0 & 0 & N/A \\
$\mathbf{7 6}$ & 8.817 & 40 & 77.1 & 23.988 & N/A & 0 & 0 & N/A \\
$\mathbf{7 7}$ & 8.82 & 35 & 77.1 & 23.988 & N/A & 0 & 0 & N/A \\
$\mathbf{7 8}$ & 8.821 & 30 & 77.1 & 23.988 & N/A & 0 & 0 & N/A \\
$\mathbf{7 9}$ & 8.822 & 25 & 77.1 & 23.988 & N/A & 0 & 0 & N/A \\
$\mathbf{8 0}$ & 8.819 & 20 & 77.1 & 23.988 & N/A & 0 & 0 & N/A \\
\hline $\mathbf{8 1}$ & 8.795 & 55 & 77.1 & 23.988 & N/A & 0 & 0 & N/A \\
$\mathbf{8 2}$ & 8.7835 & 60 & 77.1 & 23.988 & N/A & 0 & 0 & N/A \\
\hline $\mathbf{8 3}$ & 8.771 & 65 & 77.1 & 23.988 & N/A & 0 & 0 & N/A \\
$\mathbf{8 4}$ & 8.758 & 70 & 77.1 & 23.988 & N/A & 0 & 0 & N/A \\
\hline
\end{tabular}



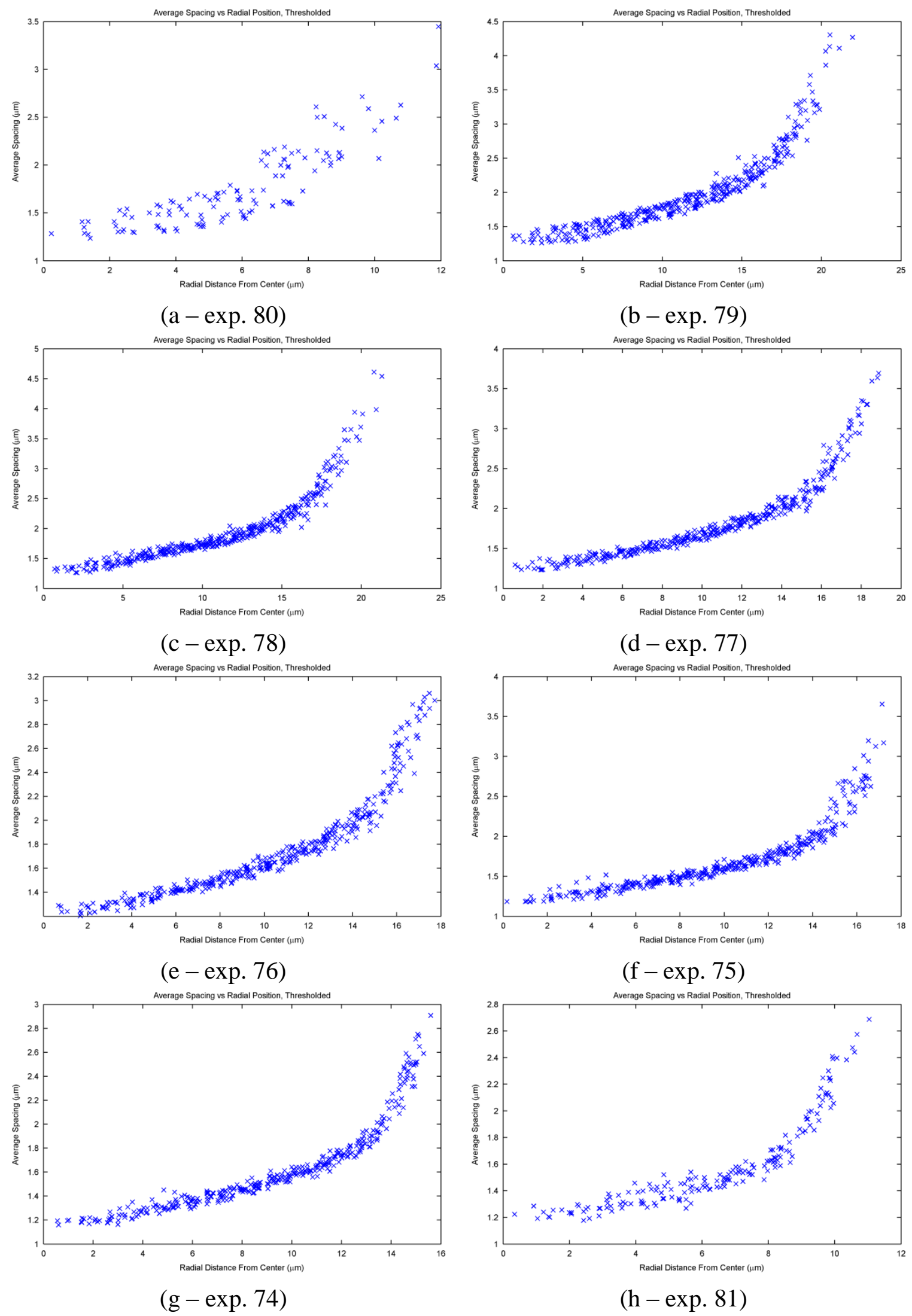

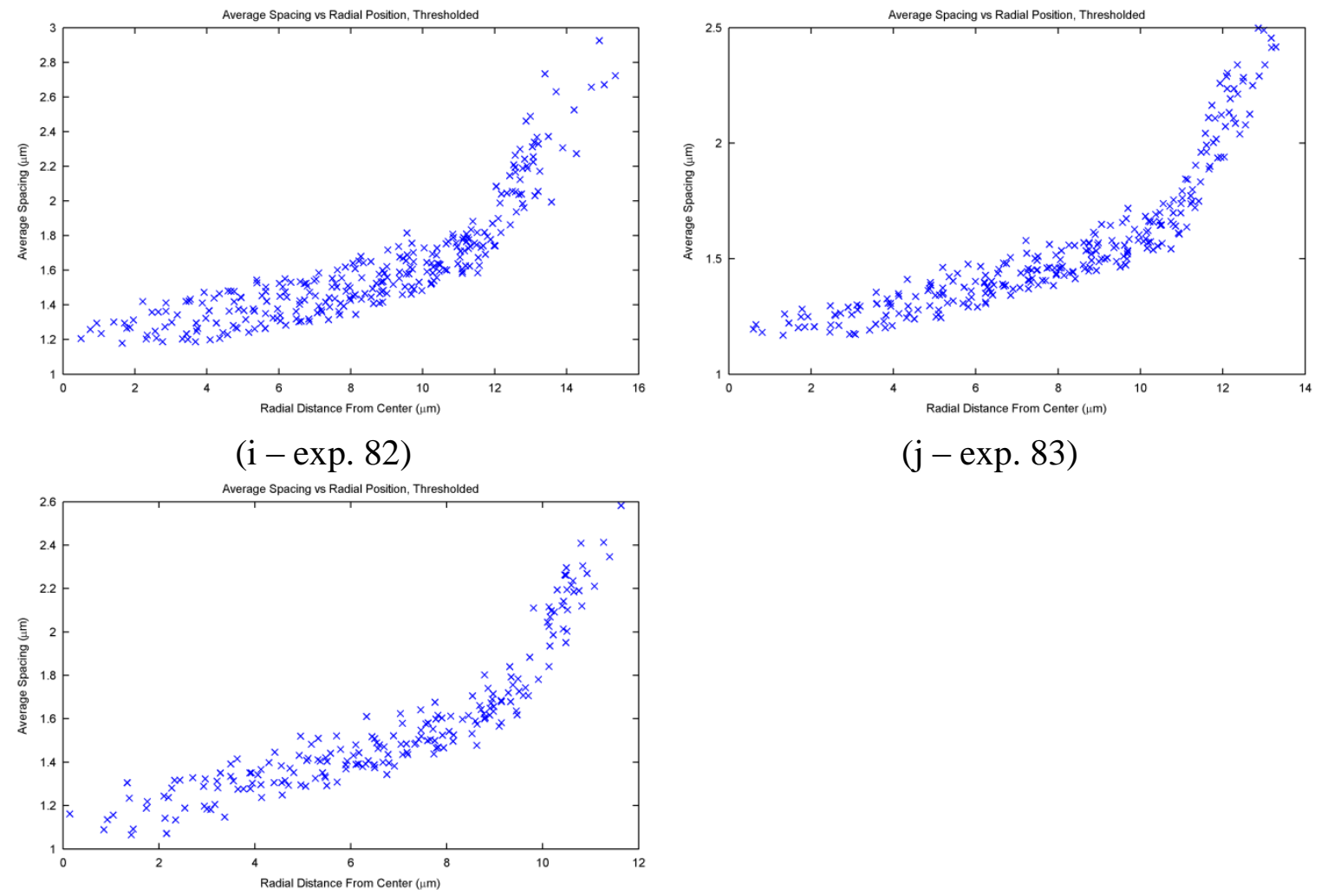

(k-exp. 84)

FIGURE A2 - Average spacing by radius in force experiments. One image used per figure. See Table AIV for parametric data. 

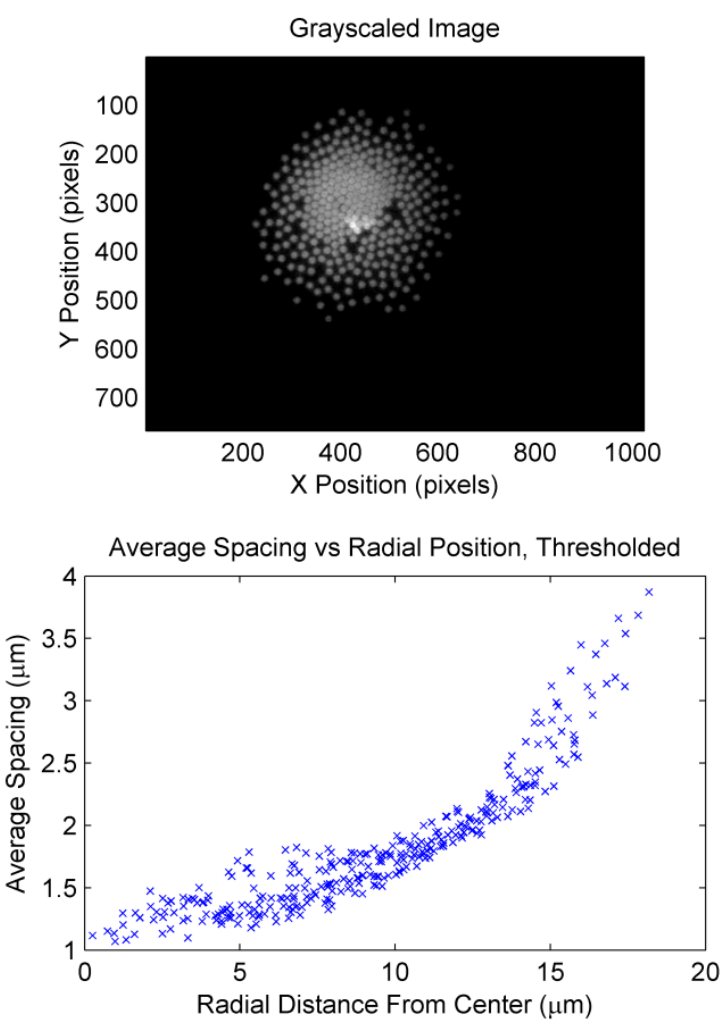

Identified Peaks Over Thresholded Image
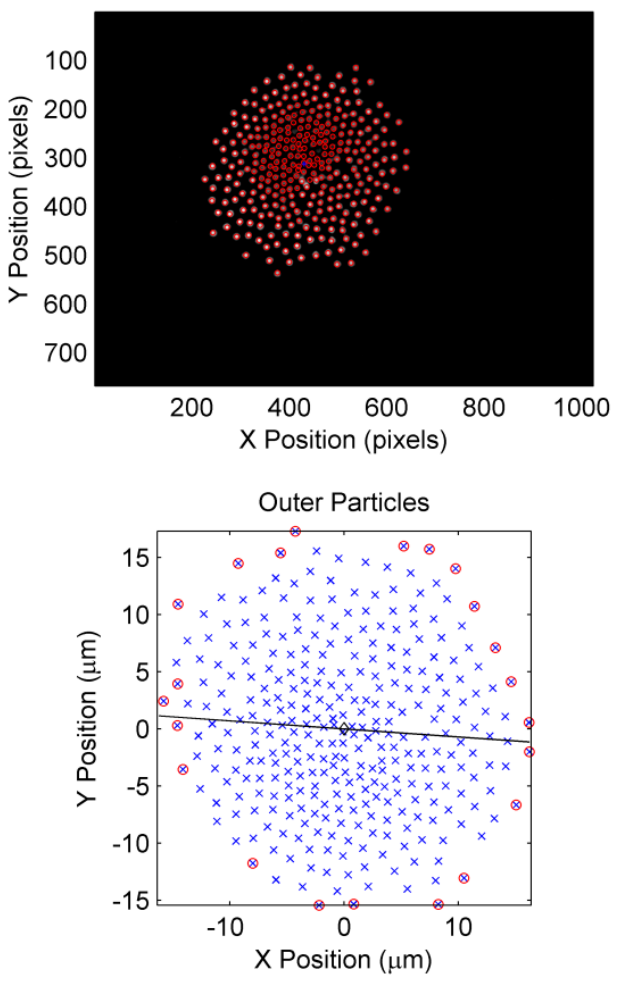

Data Set, Original vs. Thresholded

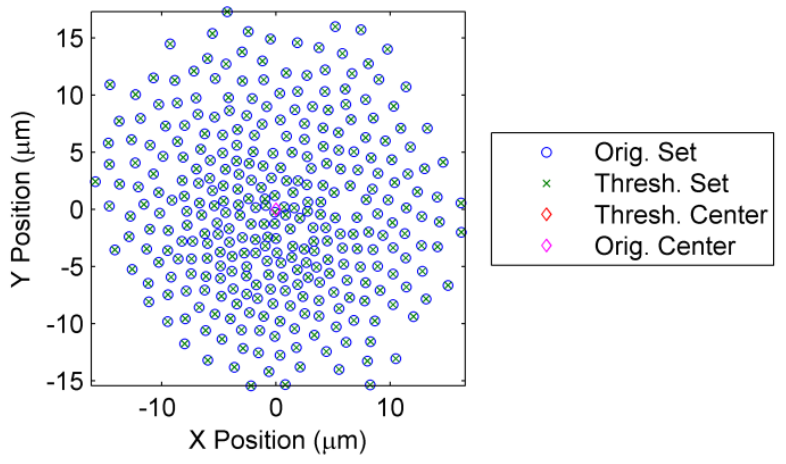

FIGURE A3 - Analysis data for experiment 1. 

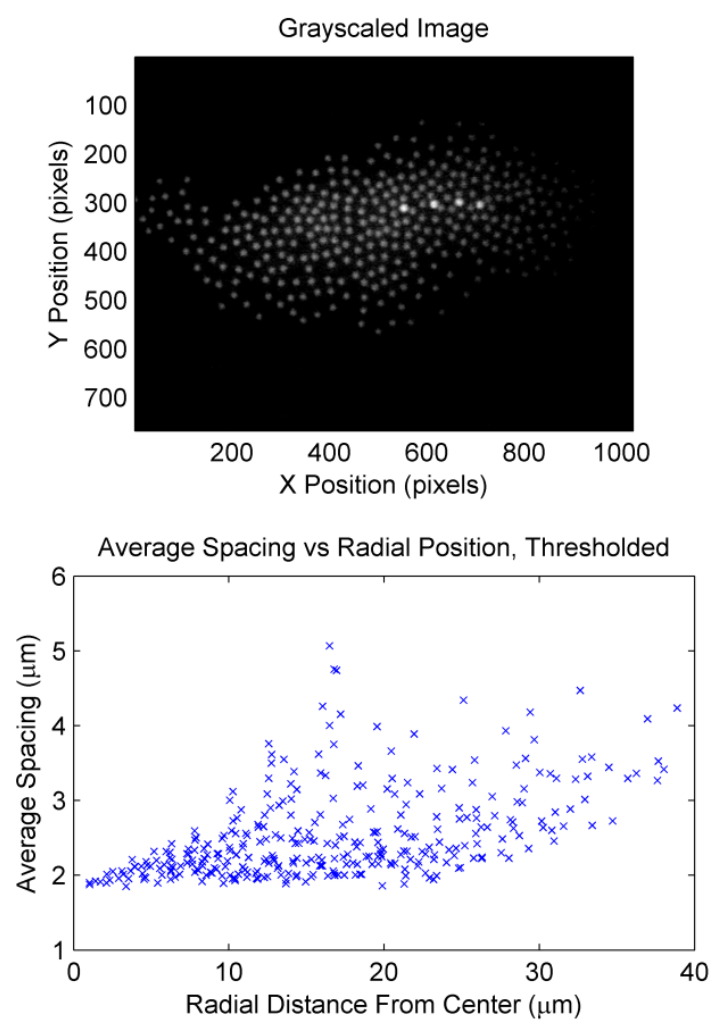

Identified Peaks Over Thresholded Image
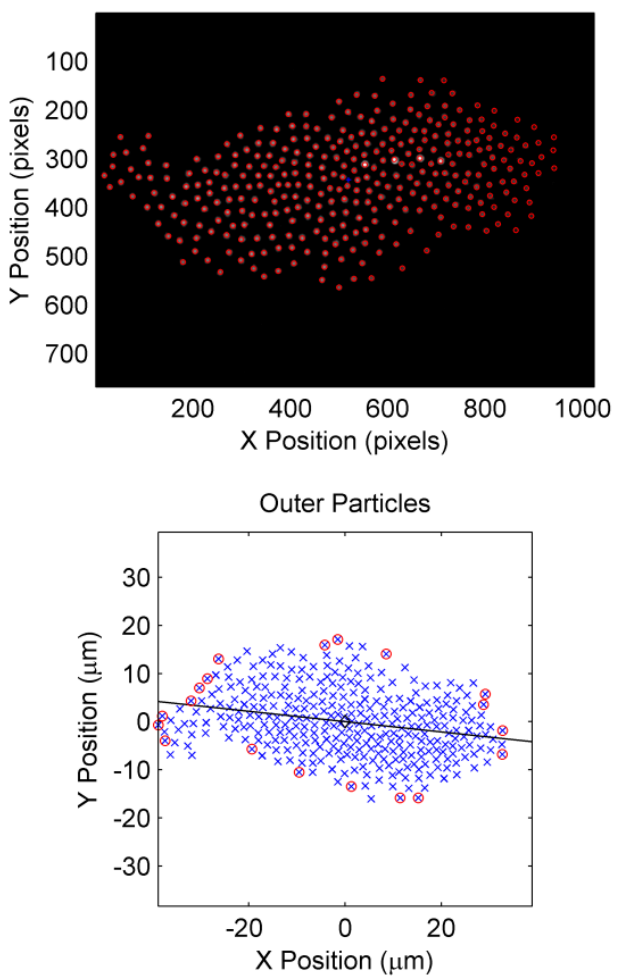

Data Set, Original vs. Thresholded

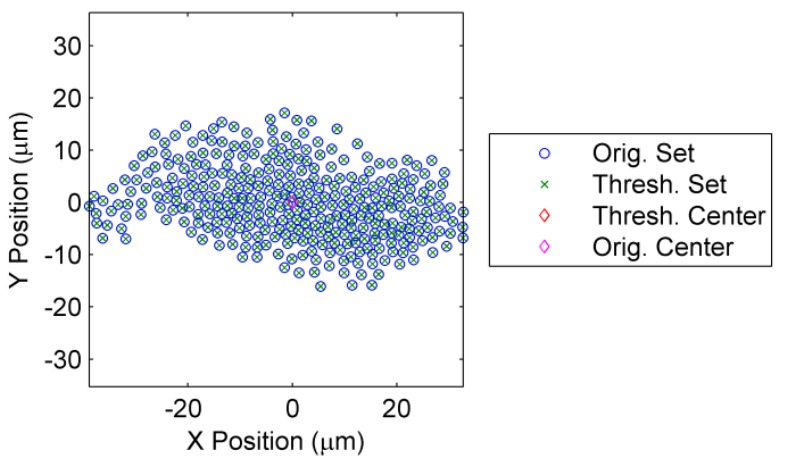

FIGURE A4 - Analysis data for experiment 8. 

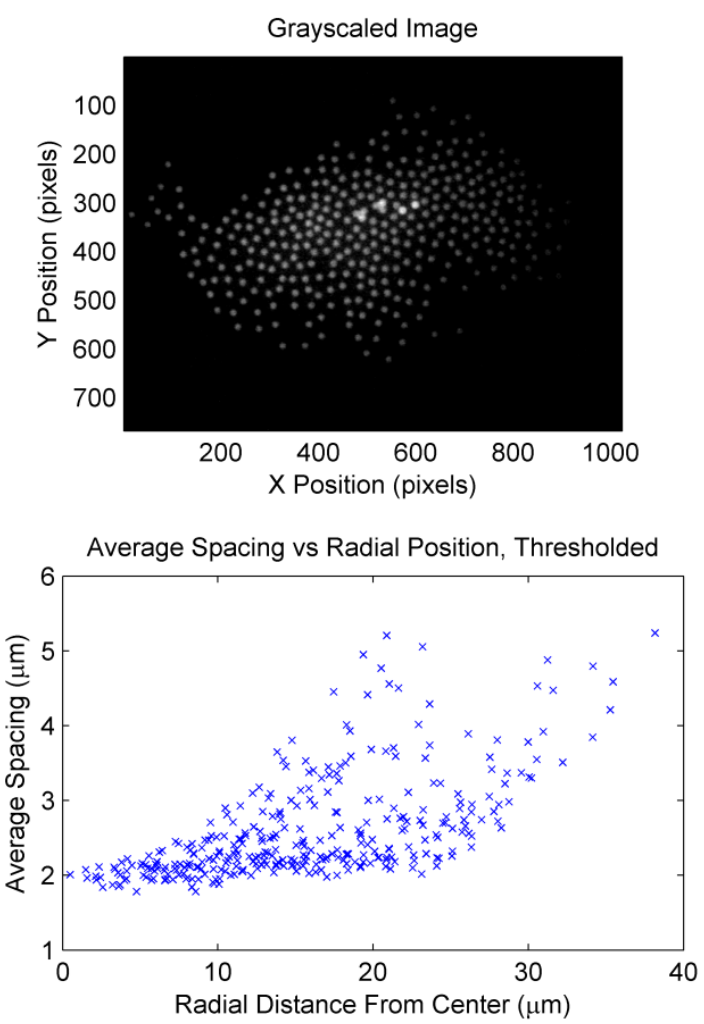

Identified Peaks Over Thresholded Image
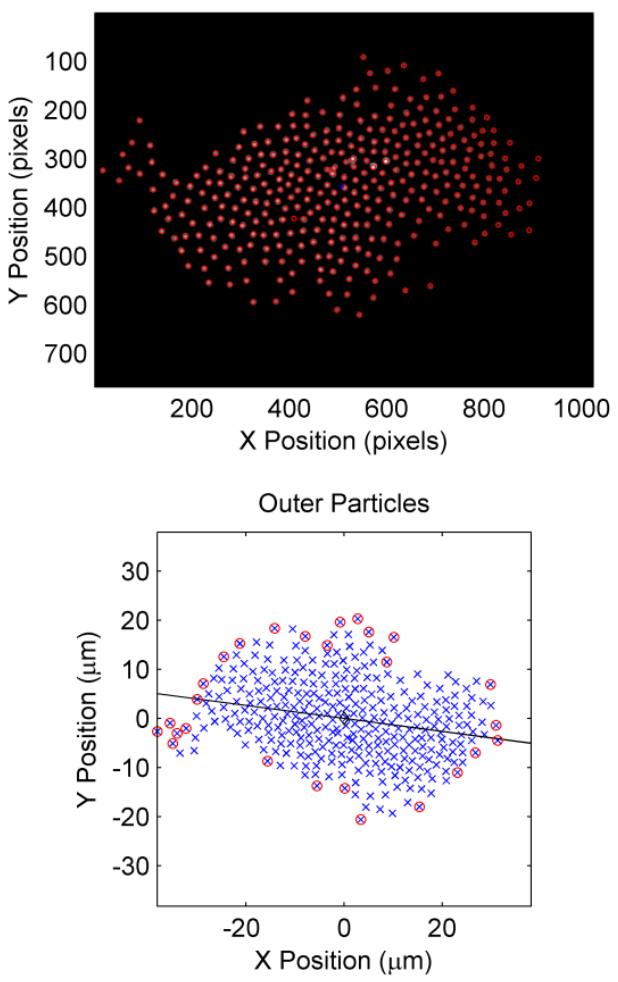

Data Set, Original vs. Thresholded

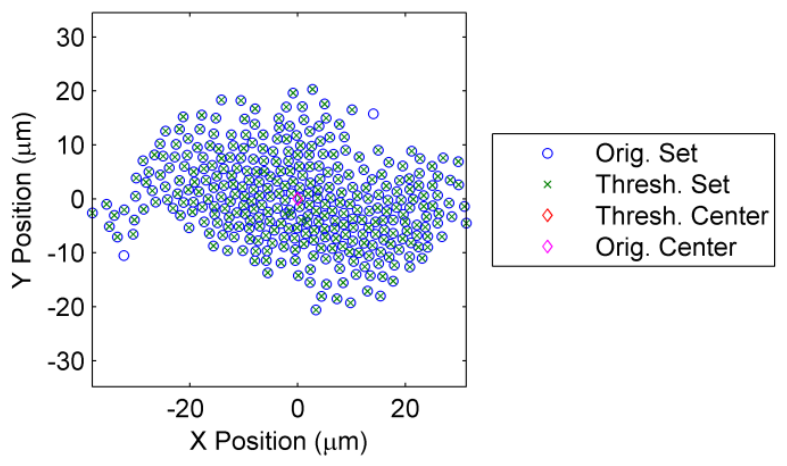

FIGURE A5 - Analysis data for experiment 9. 

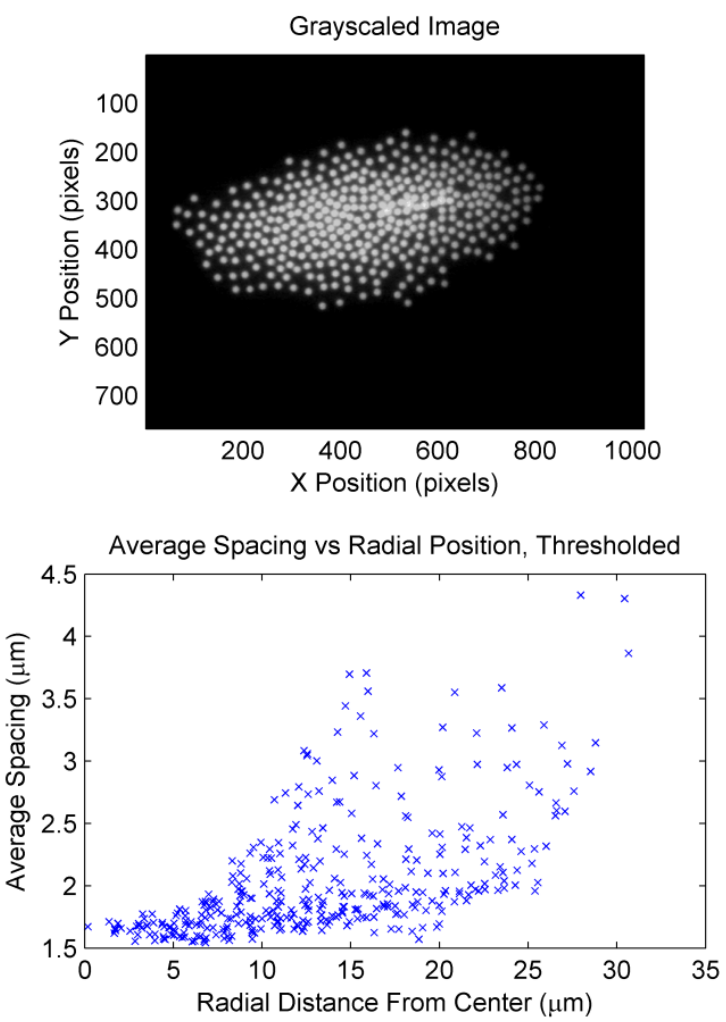

Identified Peaks Over Thresholded Image
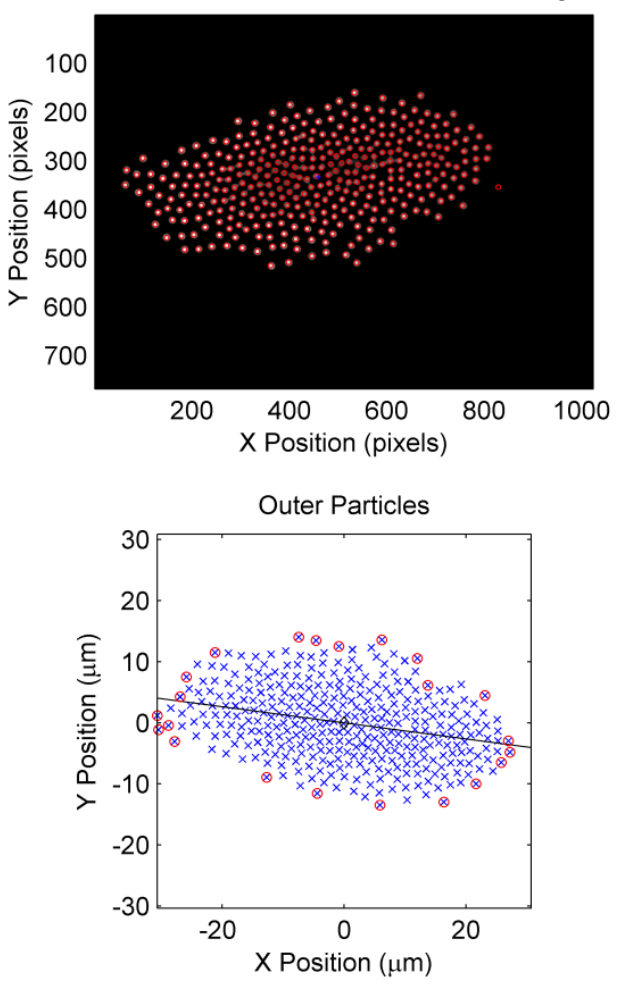

Data Set, Original vs. Thresholded

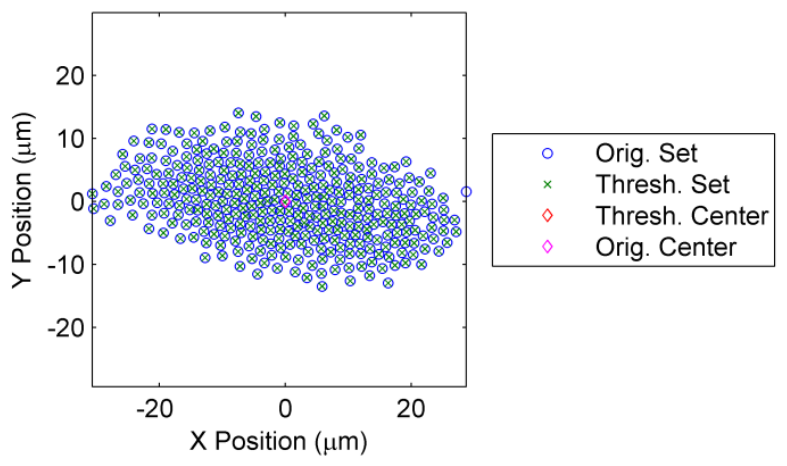

FIGURE A6 - Analysis data for experiment 19. 

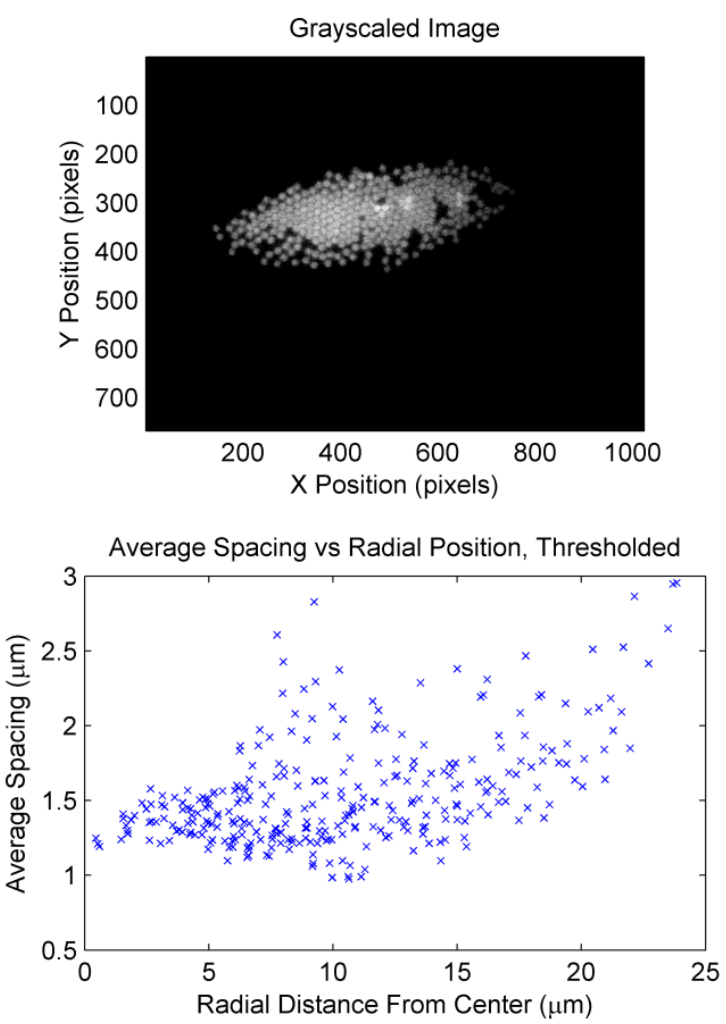

Identified Peaks Over Thresholded Image
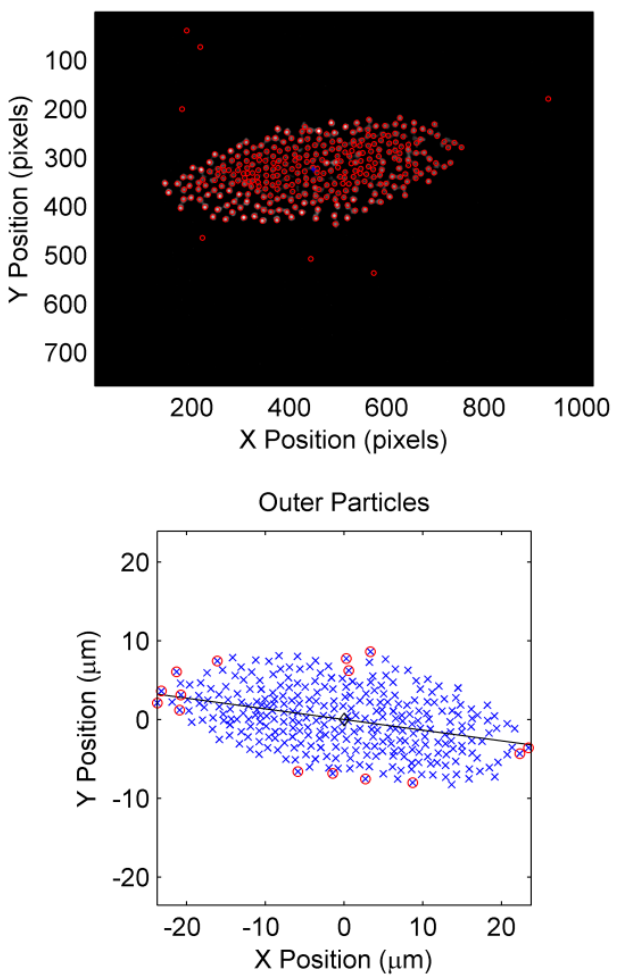

Data Set, Original vs. Thresholded

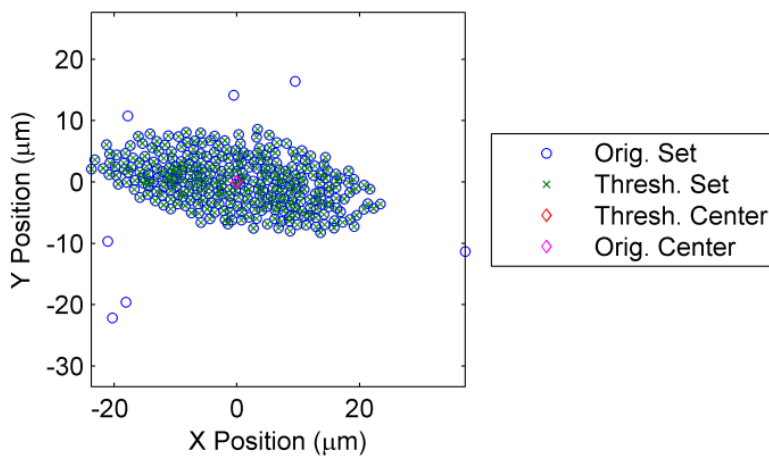

FIGURE A7 - Analysis data for experiment 29. 

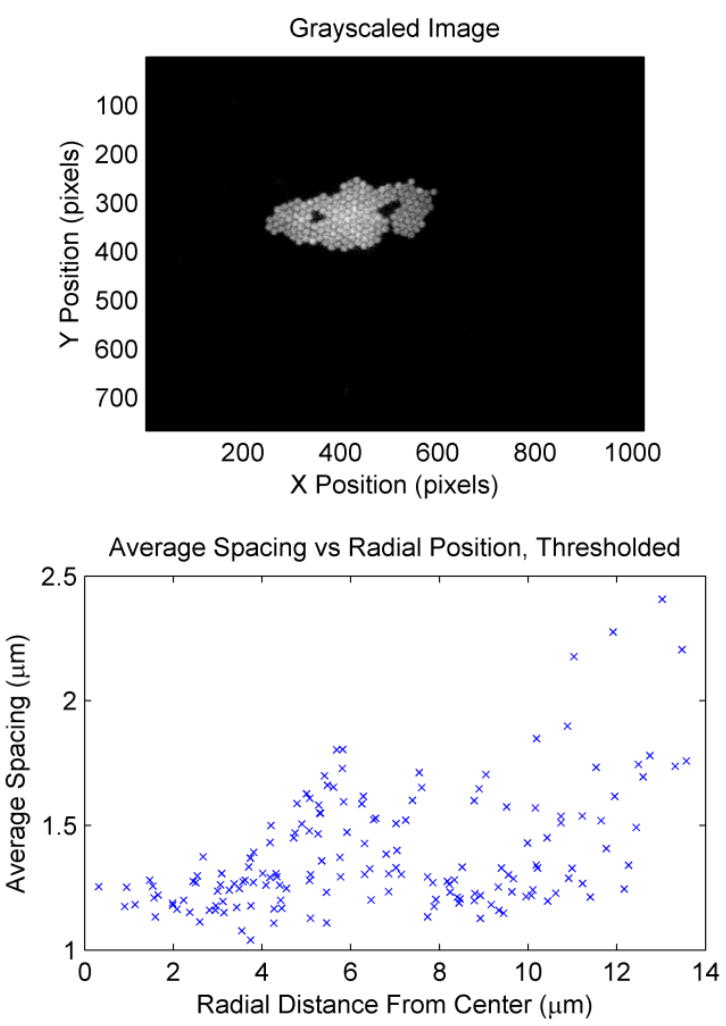

Identified Peaks Over Thresholded Image
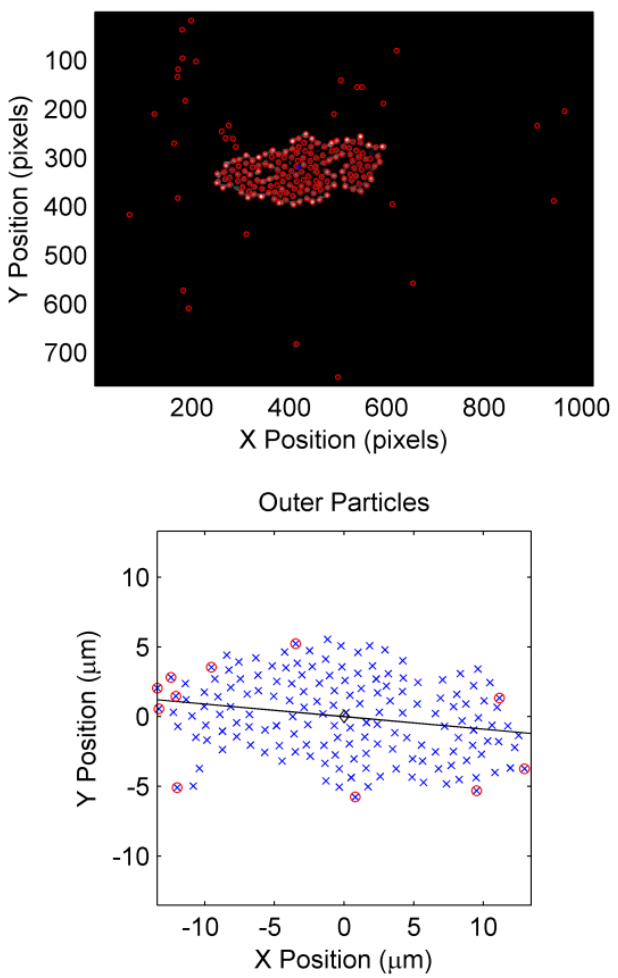

Data Set, Original vs. Thresholded

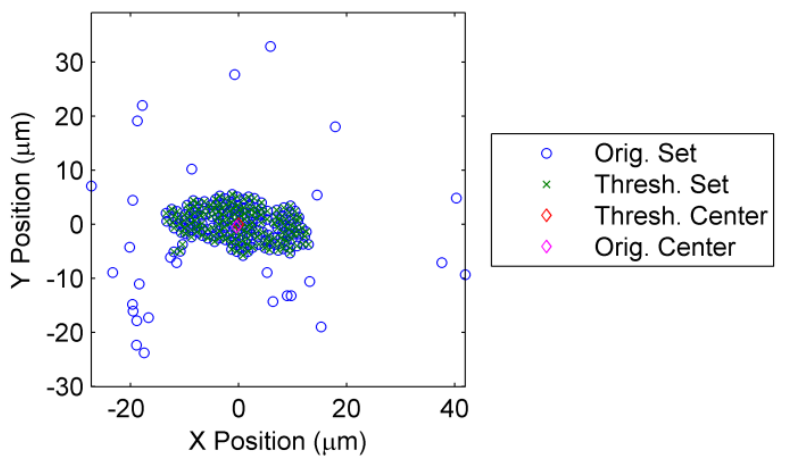

FIGURE A8 - Analysis data for experiment 35. 

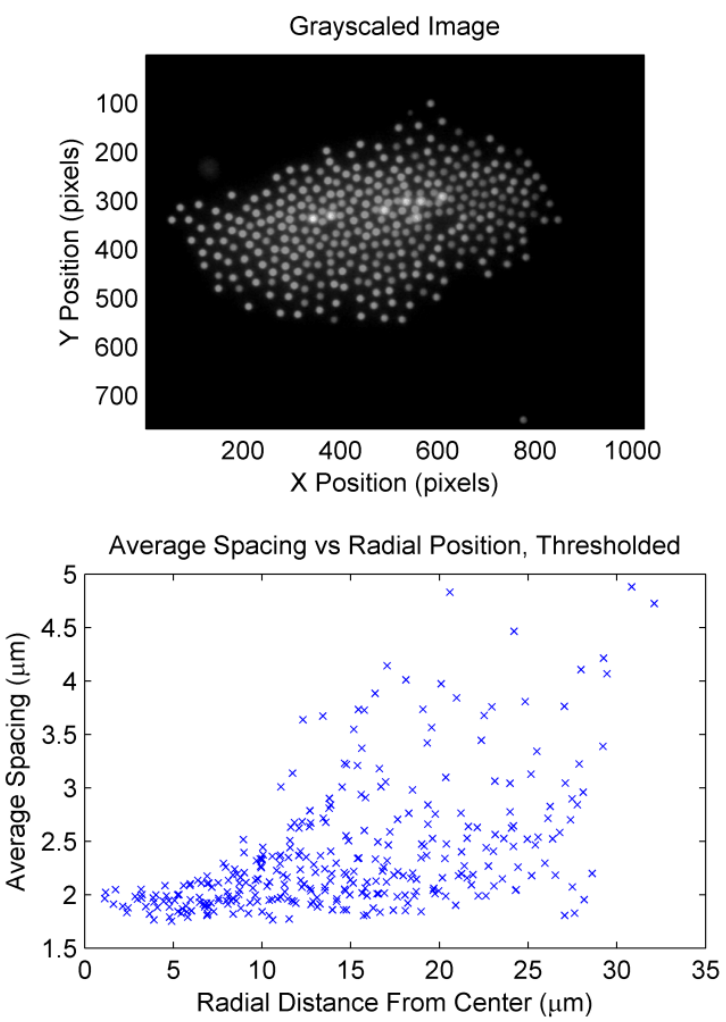

Identified Peaks Over Thresholded Image
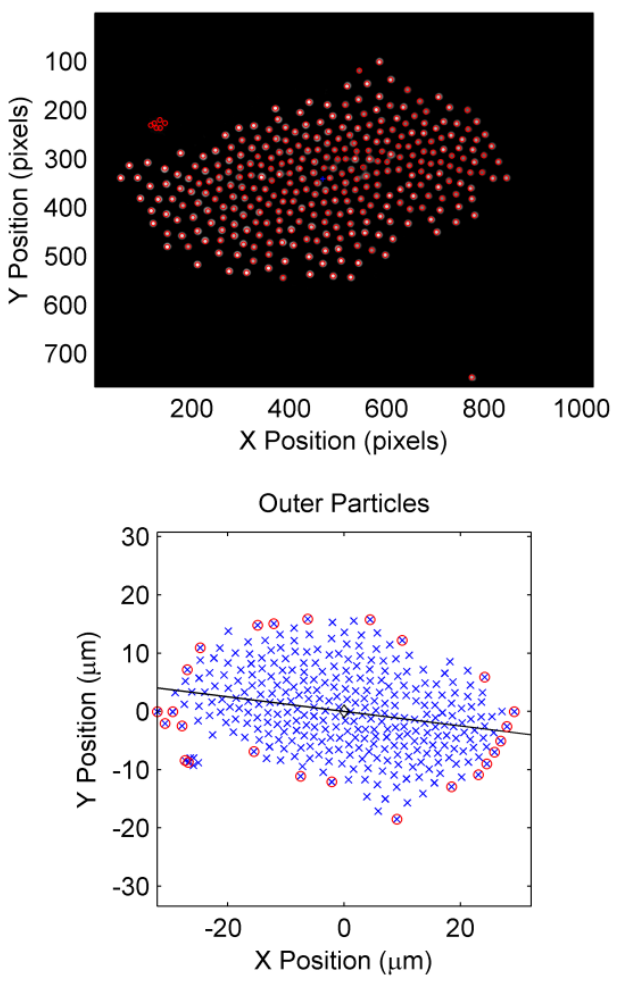

Data Set, Original vs. Thresholded

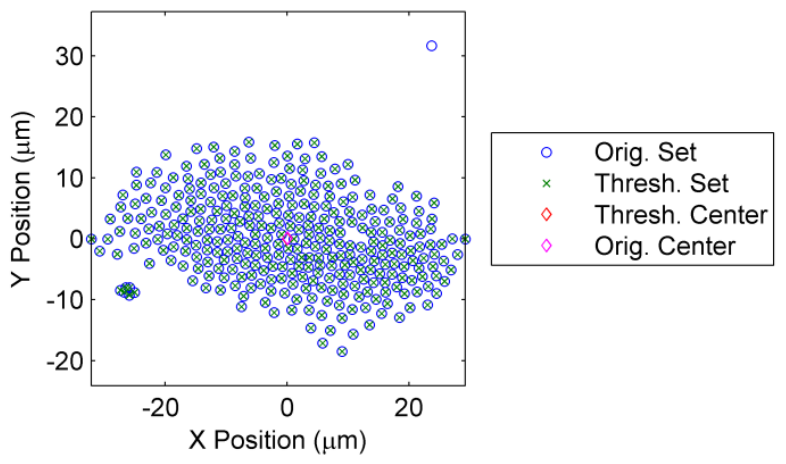

FIGURE A9 - Analysis data for experiment 36. 

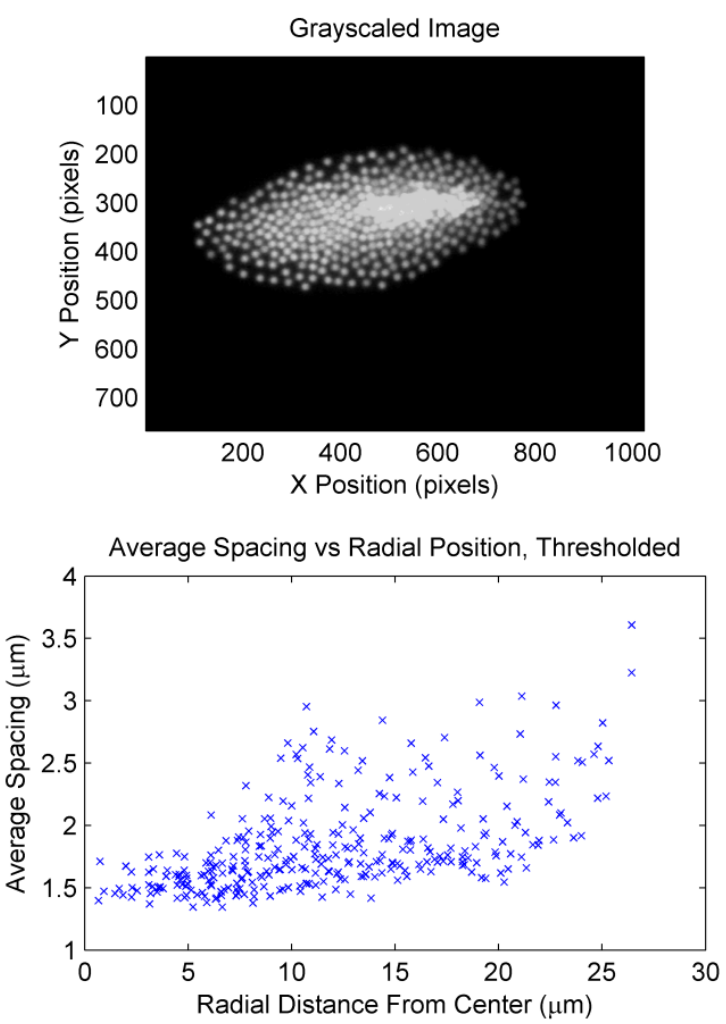

Identified Peaks Over Thresholded Image
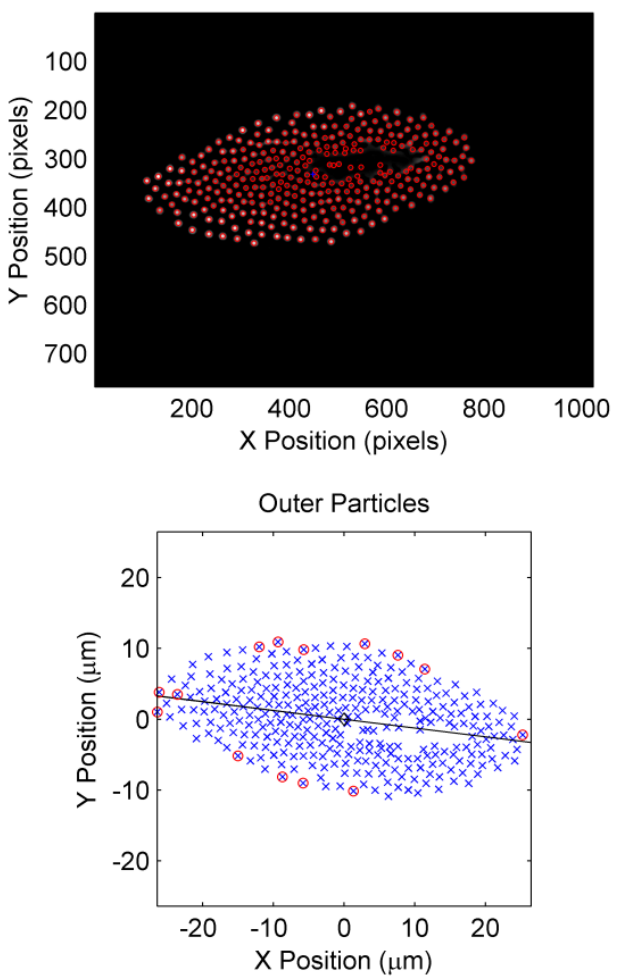

Data Set, Original vs. Thresholded

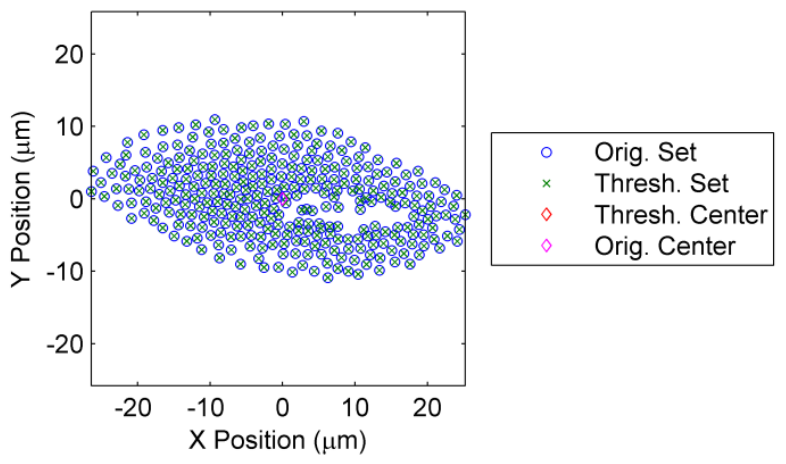

FIGURE A10 - Analysis data for experiment 52. 

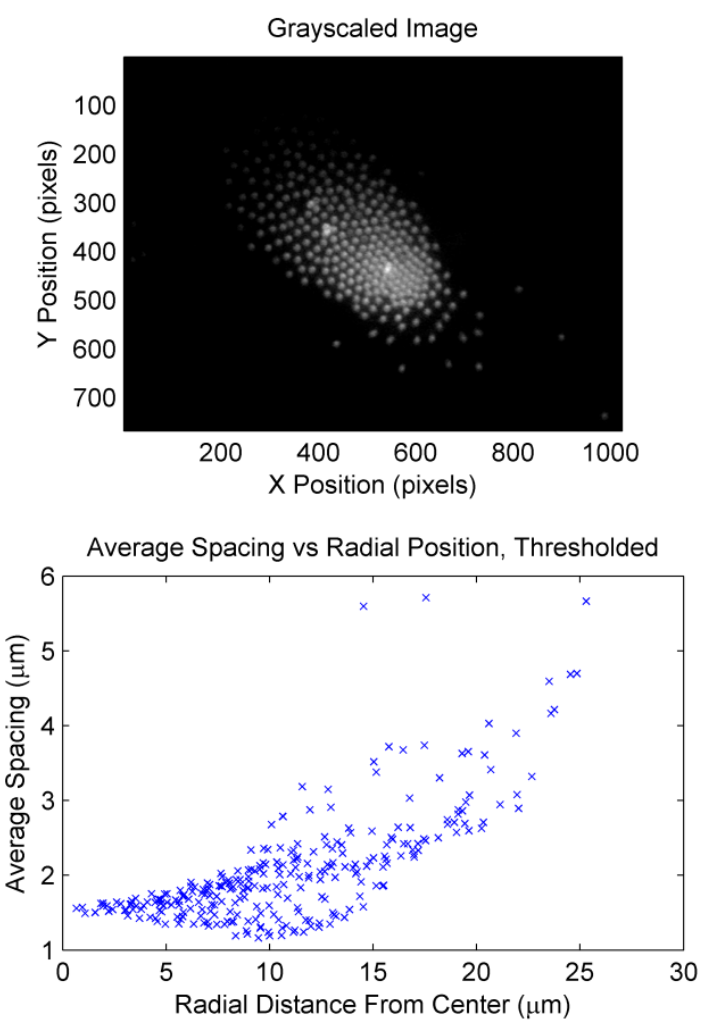

Identified Peaks Over Thresholded Image
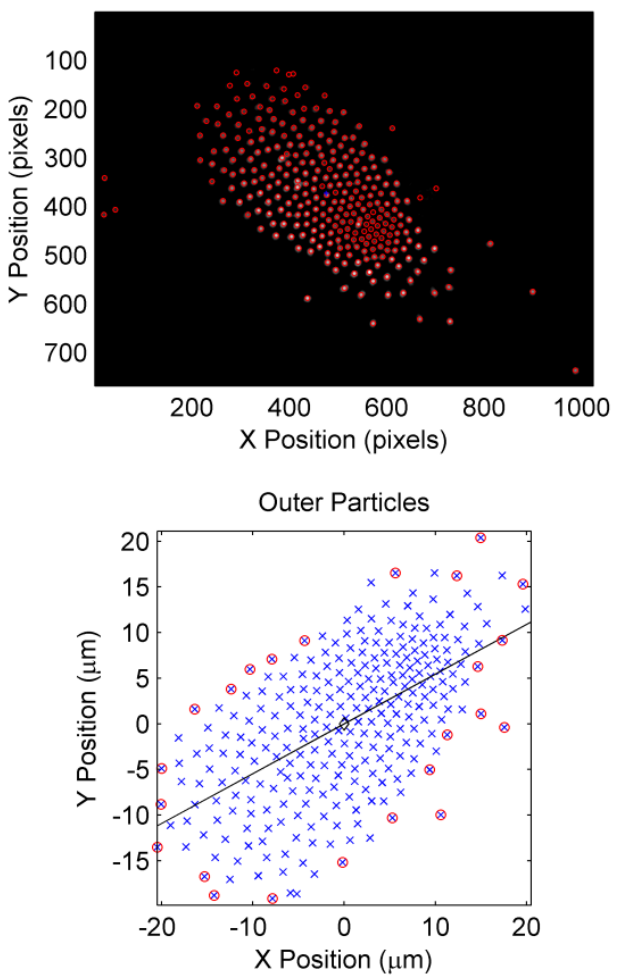

Data Set, Original vs. Thresholded

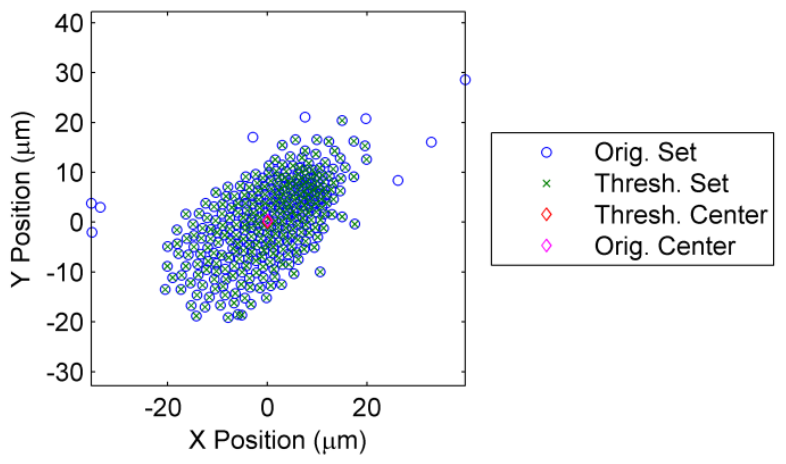

FIGURE A11 - Analysis data for experiment 53. 

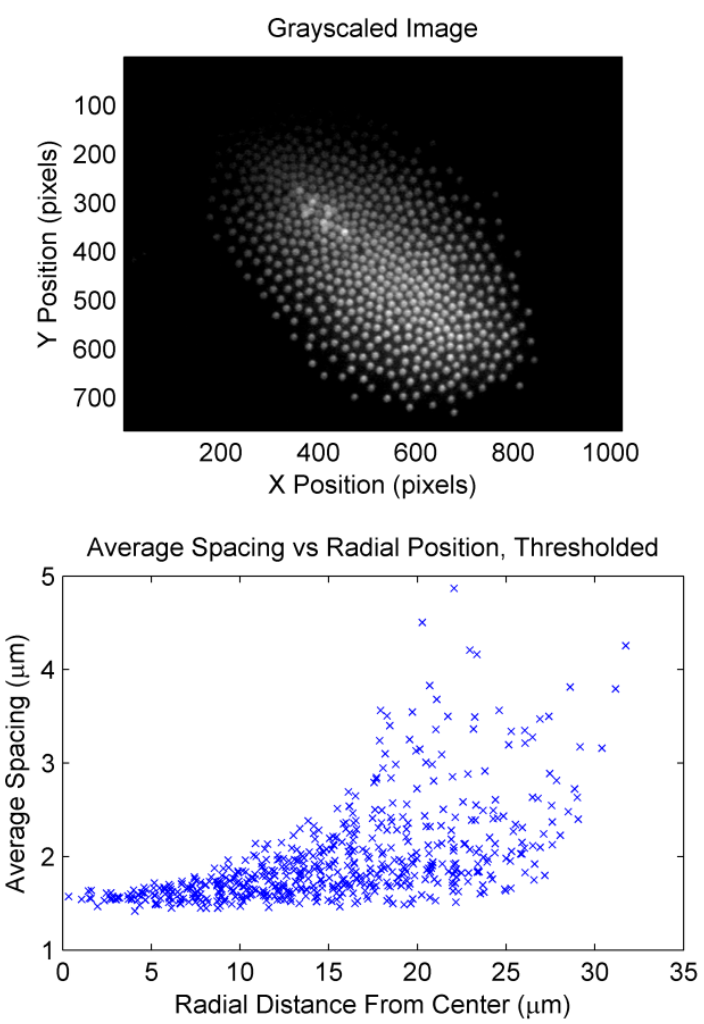

Identified Peaks Over Thresholded Image
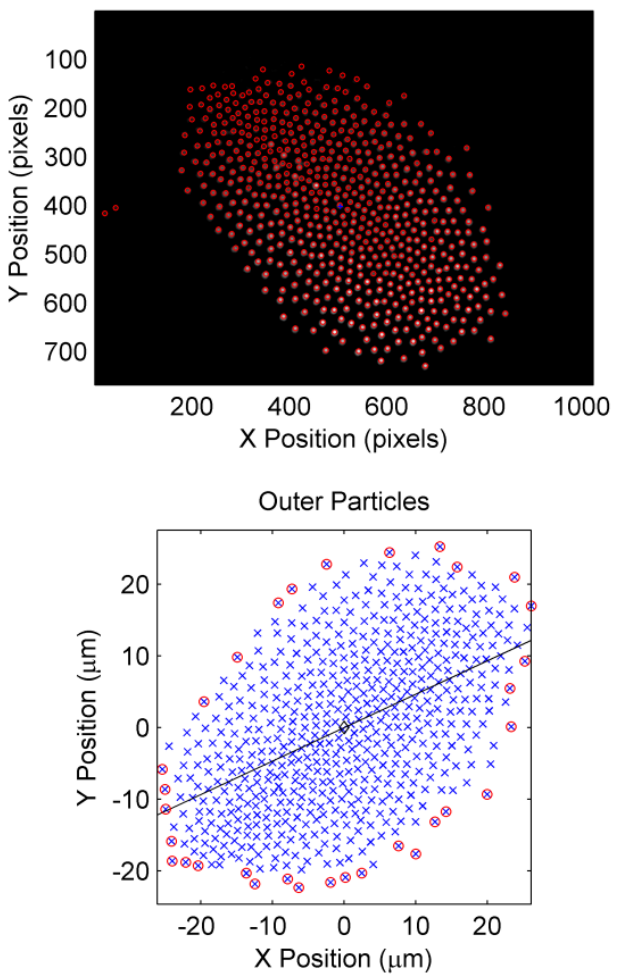

Data Set, Original vs. Thresholded

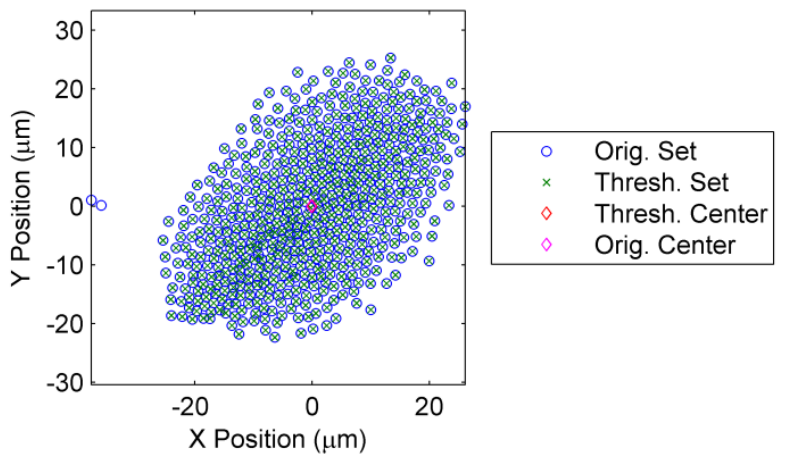

FIGURE A12 - Analysis data for experiment 64. 

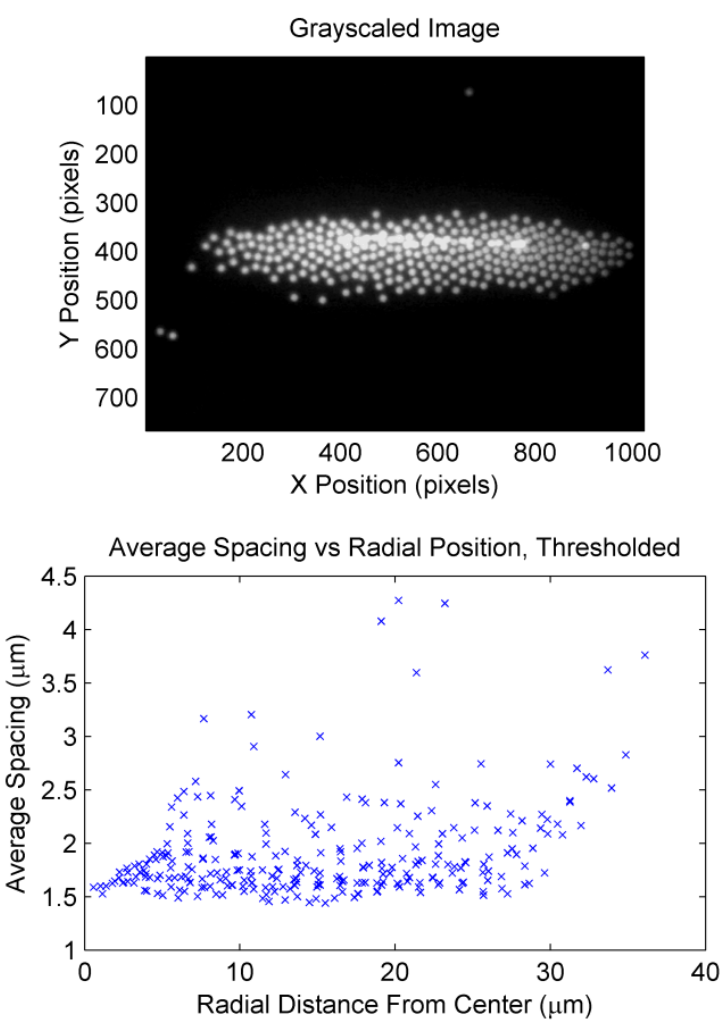

Identified Peaks Over Thresholded Image
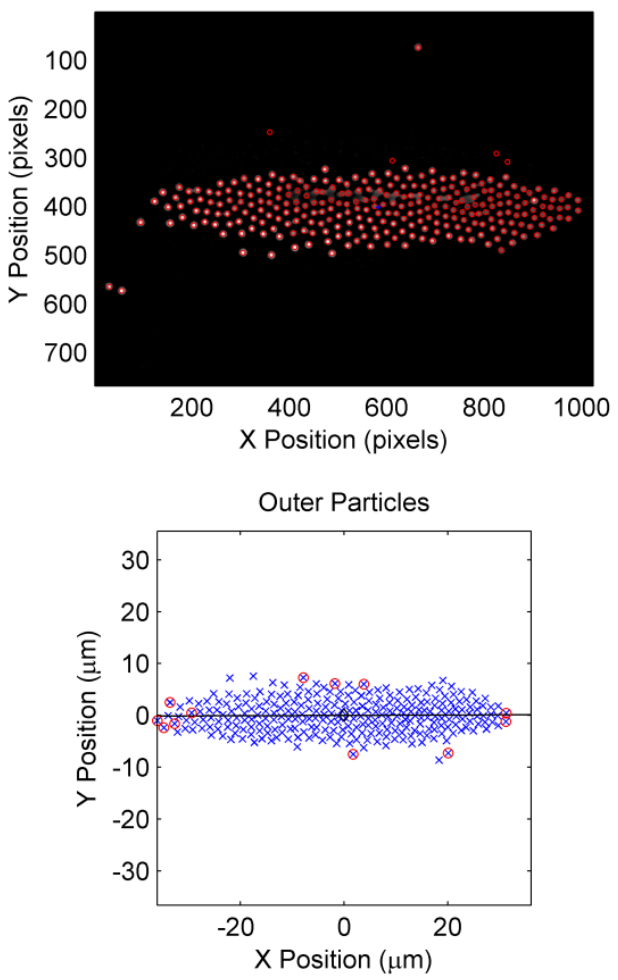

Data Set, Original vs. Thresholded

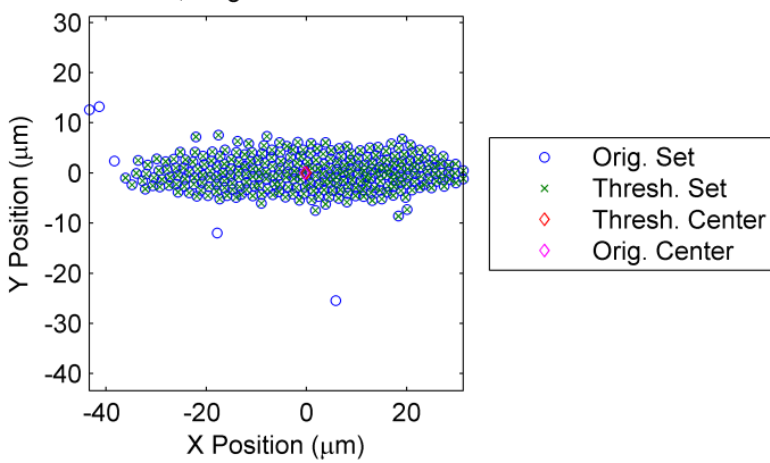

FIGURE A13 - Analysis data for experiment 65. 

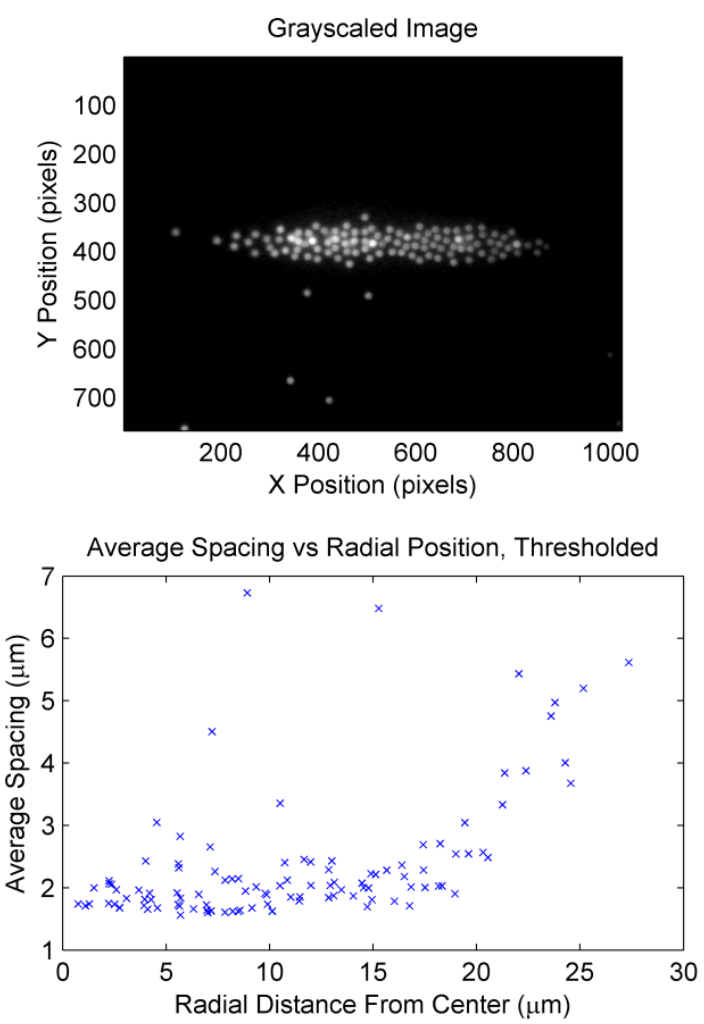

Identified Peaks Over Thresholded Image
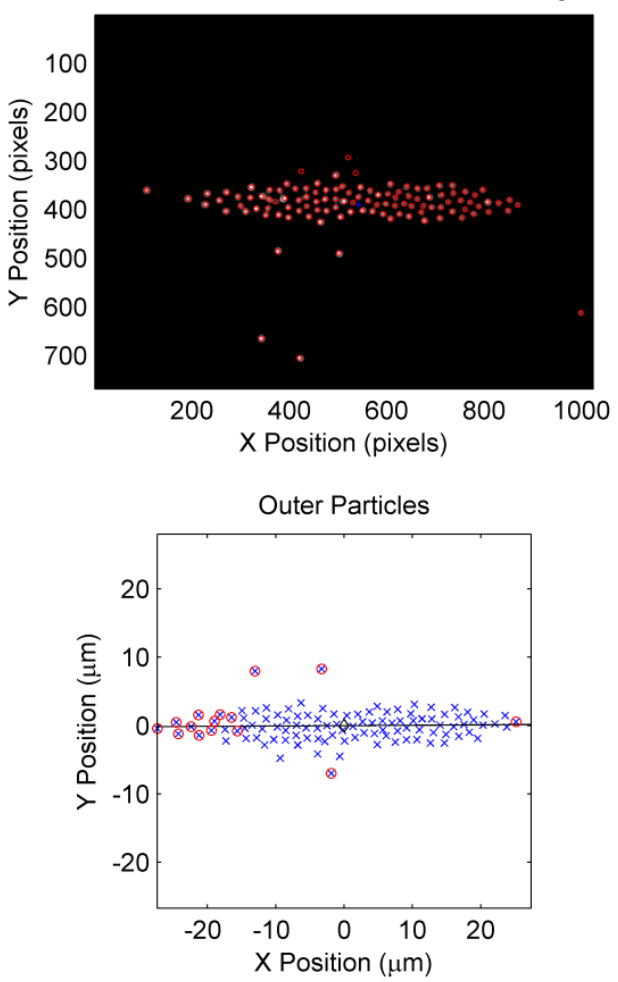

Data Set, Original vs. Thresholded

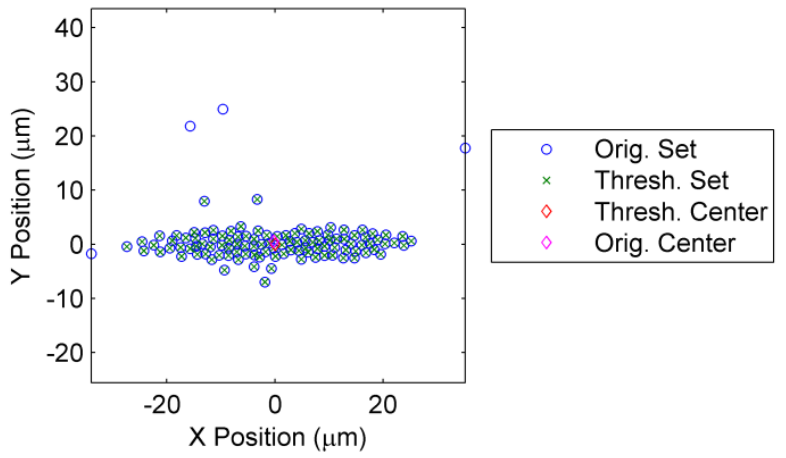

FIGURE A14 - Analysis data for experiment 69. 


\section{REFERENCES CITED}

1 Agilent. (2006). Basics of Measuring the Dielectric Properties of Material (pp. 32). USA.

2 Bhat, R. B., and Snehashish Chakraverty. (2004). Numerical Analysis in Engineering. . Pangbourne: Alpha Science International.

3 Colvin, V. L. (2001). From Opals to Optics: Colloidal Photonic Crystals. MRS Bulletin.

4 Dickinson, E., \& McClements, D. J. (1996). Advances In Food Colloids. London: Blackie Academic \& Professional.

5 Dunstan, D. E., \& Stokes, J. (2000). Diffusing Probe Measurements of Polystyrene Latex Particles in Polyelectrolyte Solutions: Deviations from StokesEinstein Behavior. Macromolecules, 33.

6 Encyclopædia Britannica. (2012a). Brownian Motion Encyclopadia Britannica. http://www.britannica.com/EBchecked/topic/81815/Brownian-motion.

7 Encyclopædia Britannica. (2012b). Colloid Encyclopadia Britannica. http://www.britannica.com/EBchecked/topic/125898/colloid.

8 Encyclopædia Britannica. (2012c). Sol Encyclopadia Britannica. http://www.britannica.com/EBchecked/topic/552808/sol.

9 Fagan, J. A., Sides, P. J., \& Prieve, D. C. (2002). Vertical Oscillatory Motion of a Single Colloidal Particle Adjacent to an Electrode in an ac Electric Field. Langmuir, 18(21), 7810-7820. doi: 10.1021/la0257211

10 Fagan, J. A., Sides, P. J., \& Prieve, D. C. (2004). Vertical Motion of a Charged Colloidal Particle near an AC Polarized Electrode with a Nonuniform Potential Distribution: Theory and Experimental Evidence. Langmuir, 20(12), 4823-4834. doi: $10.1021 / 1 \mathrm{a} 036022 \mathrm{r}$

11 Fagan, J. A., Sides, P. J., \& Prieve, D. C. (2005). Evidence of Multiple Electrohydrodynamic Forces Acting on a Colloidal Particle near an Electrode Due to an Alternating Current Electric Field. Langmuir, 21(5), 1784-1794. doi: 10.1021/la048076m

12 Hiemenz, P. C. (1997). Principles of colloid and surface chemistry. New York: M. Dekker.

13 Jiang, P., Bertone, J. F., Hwang, K. S., \& Colvin, V. L. (1999). Single-Crystal Colloidal Multilayers of Controlled Thickness. Chemistry of Materials, 11(8), 2132-2140. doi: 10.1021/cm990080+ 
14 Kim, S., Asmatulu, R., Marcus, H. L., \& Papadimitrakopoulos, F. (2011). Dielectrophoretic assembly of grain-boundary-free 2D colloidal single crystals. Journal of Colloid and Interface Science, 354(2), 448-454. doi: 10.1016/j.jcis.2010.11.023

15 Kirby, B. J. (2010). Micro- and Nanoscale Fluid Mechanics. 32 Avenue of the Americas, New York, NY 10013-2473, USA: Cambridge University Press.

16 Kumar, A., Kwon, J.-S., Williams, S. J., Green, N. G., Yip, N. K., \& Wereley, S. T. (2010). Optically Modulated Electrokinetic Manipulation and Concentration of Colloidal Particles near an Electrode Surface. Langmuir, 26(7), 5262-5272. doi: 10.1021/la904661y

17 Lide, D. R. (1993). CRC Handbook of Chemistry and Physics (74th ed.). Boca Raton: CRC.

18 Lyklema, J. (1991). Fundamentals of Interface and Colloid Science Volume I: Fundamentals (Vol. 1): Academic Press.

19 Morgan, \& Green, N. G. (2003). AC Electrokinetics: colloids and nanoparticles. Baldock, Hertfordshire, England: Research Studies Press LTD.

20 Munson, Y., Okiishi, Heubsch. (2009). Fundamentals of Fluid Mechanics. United States of America: Don Fowley.

21 Nadal, F., Argoul, F., Hanusse, P., Pouligny, B., \& Ajdari, A. (2002). Electrically induced interactions between colloidal particles in the vicinity of a conducting plane. Physical Review E, 65(6). doi: 10.1103/PhysRevE.65.061409

22 Novotny, L., Bian, R. X., \& Xie, X. S. (1997). Theory of Nanometric Optical Tweezers. Physical Review Letters, 79(4), 645-648.

23 Pieranski, P. (1983). Colloidal Crystals. [Review]. Contemporary Physics, 24(1), 25-73. doi: 10.1080/00107518308227471

24 Rengarajan, R., Jiang, P., Colvin, V., \& Mittleman, D. (2000). Optical properties of a photonic crystal of hollow spherical shells. Applied Physics Letters, 77(22).

25 Rengarajan, R., Jiang, P., Larrabee, D. C., Colvin, V. L., \& Mittleman, D. M. (2001). Colloidal photonic superlattices. Physical Review B, 64(205103).

26 Ristenpart, W. D., Aksay, I. A., \& Saville, D. A. (2004). Assembly of colloidal aggregates by electrohydrodynamic flow: Kinetic experiments and scaling analysis. Physical Review E, 69(2). doi: 10.1103/PhysRevE.69.021405

27 Rodriguez, M. A., \& Armstrong, D. W. (2004). Separation and analysis of colloidal/nano-particles including microorganisms by capillary electrophoresis: a fundamental review. Journal of Chromatography B, 800(1-2), 7-25. doi: 10.1016/j.jchromb.2003.09.060

28 Santana-Solano, J., Wu, D. T., \& Marr, D. W. (2006). Direct measurement of colloidal particle rotation and field dependence in alternating current electrohydrodynamic flows. Langmuir, 22(13), 5932-5936. doi: 10.1021/la060270g

29 Scientific, T. F. (2012). Thermo Scientific* Fluoro-Max Dyed Red Aqueous Fluorescent Particles Retrieved 6/19/2012, 2012, from http://www.fishersci.com/ecomm/servlet/fsproductdetail? catalogId=-

$1 \&$ productId $=10898266 \&$ langId $=-$

$\underline{1 \& \text { store } I d=10652 \& \text { distype }=2 \& \text { isChemical }=\text { false } \& \text { fromSearch }=0}$ 
30 Velasco, V., Work, A., \& Williams, S. J. (2012). Electrokinetic Concentration and Patterning of Colloids with a Scanning Laser. Electrophoresis.

31 Williams, S. J., Kumar, A., \& Wereley, S. T. (2008). Electrokinetic patterning of colloidal particles with optical landscapes. Lab on a Chip, 8(11), 1879. doi: 10.1039/b810787d

32 Williamson, C. H. K. (1988). Defining a universal and continuous StrouhalReynolds number relationship for the laminar vortex shedding of a circular cylinder. Physics of Fluids, 31(2742), 3. doi: 10.1063/1.866978

33 Zhang, J., Li, Y., Zhang, X., \& Yang, B. (2010). Colloidal Self-Assembly Meets Nanofabrication: From Two-Dimensional Colloidal Crystals to Nanostructure Arrays. Advanced Materials, 22.

34 Zheng, F. (2002). Thermophoresis of spherical and non-spherical particles: a review of theories and experiments. Advances in Colloid and Interface Science, 97(1-3), 255-278. doi: 10.1016/s0001-8686(01)00067-7

35 Zhou, H., White, L. R., \& Tilton, R. D. (2005). Lateral separation of colloids or cells by dielectrophoresis augmented by AC electroosmosis. Journal of Colloid and Interface Science, 285(1), 179-191. doi: 10.1016/j.jcis.2004.11.040 


\section{Andrew H. Work Jr., EIT \\ 1022 N. Clark Boulevard \\ Clarksville, IN 47129 \\ andrew@andrewwork.com}

EDUCATION M. Eng. in Mechanical Engineering - GPA 4.000/4

Expected July 2012

B.S. in Mechanical Engineering - GPA 3.465/4

May 2011

J.B. Speed School of Engineering, UofL, Louisville, KY

High School Diploma

May 2007

Christian Academy of Louisville, Louisville, KY

$\begin{array}{lll}\text { SKILLS/ } & \bullet \text { Differential Equations } & \bullet \text { C\# Programming } \\ \text { COURSEWORK } & \bullet \text { System Dynamics } & \bullet \text { HTMLCSS } \\ & \text { - Experimental Stress Analysis } & \bullet \text { Data Acquisition } \\ & \text { - Fiber Reinforced Composites } & \bullet \text { Solid Works } \\ & \text { - VBA Programming for Excel } & \end{array}$

EMPLOYMENT GTA, University of Louis ville, Louis ville, KY

HISTORY

- GTA for M.E. Department

- Develop individual labs

- Run lab sessions

- Grade papers

Math Tutor, University of Louis ville, Louis ville, KY

- Calc. 1 SI Leader

- Ran help session

- Classroomenvironment

Engineering Co-op, Hamilton Sundstrand, Rockford, IL

- Quality Engineering

- VBA programming

- Access Database

- Documentation

Engineering Co-op, Hamilton Sundstrand, Rockford, IL

- Electronics Packaging

- EC Creation

- VBA programming

- Cross-department website

Engineering Co-op, Hamilton Sundstrand, Rockford, IL

- Manufacturing Engineering

- Data Entry

- Tool design

- JIS modification/creation

ACTIVITIES

- Micropump research

/HONORS
- Pi Tau Sigma VP
- Micropump fabrication

- Trustee's Scholarship
May 2011- May 2012

Aug - Dec 2010

May 2010 - July 2010

Aug 2009 - Dec 2009

Jan 2009 - May 2009 